\title{
Cognitive processes in adults with ADHD
}

Citation for published version (APA):

Marchetta, N. D. J. (2007). Cognitive processes in adults with ADHD. [Doctoral Thesis, Maastricht University]. Datawyse / Universitaire Pers Maastricht. https://doi.org/10.26481/dis.20071129nm

Document status and date:

Published: 01/01/2007

DOI:

10.26481/dis.20071129nm

Document Version:

Publisher's PDF, also known as Version of record

\section{Please check the document version of this publication:}

- A submitted manuscript is the version of the article upon submission and before peer-review. There can be important differences between the submitted version and the official published version of record.

People interested in the research are advised to contact the author for the final version of the publication, or visit the DOI to the publisher's website.

- The final author version and the galley proof are versions of the publication after peer review.

- The final published version features the final layout of the paper including the volume, issue and page numbers.

Link to publication

\footnotetext{
General rights rights.

- You may freely distribute the URL identifying the publication in the public portal. please follow below link for the End User Agreement:

www.umlib.nl/taverne-license

Take down policy

If you believe that this document breaches copyright please contact us at:

repository@maastrichtuniversity.nl

providing details and we will investigate your claim.
}

Copyright and moral rights for the publications made accessible in the public portal are retained by the authors and/or other copyright owners and it is a condition of accessing publications that users recognise and abide by the legal requirements associated with these

- Users may download and print one copy of any publication from the public portal for the purpose of private study or research.

- You may not further distribute the material or use it for any profit-making activity or commercial gain

If the publication is distributed under the terms of Article $25 \mathrm{fa}$ of the Dutch Copyright Act, indicated by the "Taverne" license above, 


\section{COGNITIVE PROCESSES IN}

\section{ADULTS WITH ADHD}

Neuropsych Publishers, Maastricht 
(C) N. D. J. Marchetta, Tongeren/Maastricht 2007

ISBN 978-90-75578-35-2

Cover design: N. D. J. Marchetta and Datawyse B. V., Maastricht

Illustration: painting by Ossiala

Production: $\quad$ Datawyse B. V. I Universitaire Pers Maastricht

Lay-out: $\quad$ Femie Kengen

Neuropsych Publishers is a non-profit organisation, which aims at promoting the science of 'Brain and Behavior'and improving the application of the products of this science in health care and education. Neuropsych Publishers accomplishes these aims by publishing books, dissertations and other products of scientific activity, by disseminating educational materials and publication of tests, assess-ment scales and other psychometric instruments in the field of Neuropsychology, Neuropsychiatry and other areas within the domain of Brain and Behavior.

Postal address: Neuropsych Publishers

Department of Psychiatry and Neuropsychology

Maastricht University

P.O. Box 616

6200 MD Maastricht

The Netherlands

www.np.unimaas.nl. 


\section{COGNITIVE PROCESSES IN}

\section{ADULTS WITH ADHD}

\section{PROEFSCHRIFT}

ter verkrijging van de graad van doctor aan de Universiteit Maastricht, op gezag van de Rector Magnificus, Prof. mr. G. P. M. F. Mols, volgens het besluit van het College van Decanen, in het openbaar te verdedigen op donderdag 29 november 2007 om 16.00 uur

door

Natalie Dominique Jenny Marchetta

Geboren op 22 december 1980 te Tongeren(België) 


\section{Promotor}

Prof. dr. J. Jolles

\section{Copromotor}

Dr. P. P. M. Hurks

\section{Beoordelingscommissie}

Prof. dr. F. R. J. Verhey (voorzitter)

Prof. dr. A. P. Aldenkamp

Prof. dr. A. Korenbrits

Dr. L. M. J. de Sonneville (Universiteit Leiden en VUMC Amsterdam)

Prof. dr. H. Swaab-Barneveld (Universiteit Leiden)

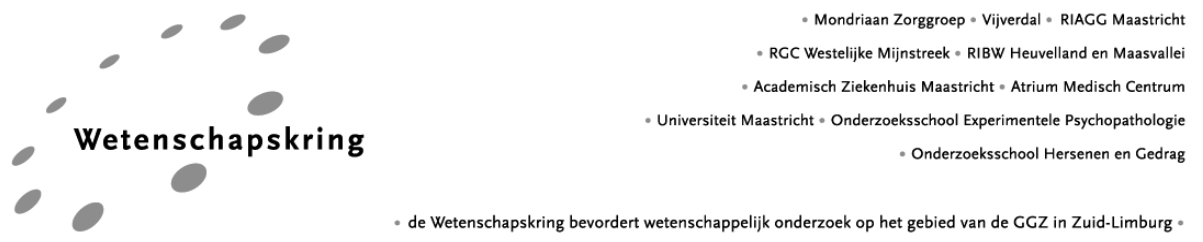

The research described in this dissertation was performed in the Maastricht Brain and Behavior Institute, Department of Psychiatry and Neuropsychology, Maastricht University, the University Hospital Maastricht, and the Psychomedical Centre Vijverdal.

Financial support for the publication of this dissertation has been kindly provided by Eli Lilly Nederland B. V., Eurocept Pharmaceuticals, Janssen-Cilag B. V., and Lundbeck B. V. 


\section{Contents}

Chapter 1 ADHD in adulthood: An introduction 7

Chapter $2 \quad$ Interference control, working memory, 27 concept shifting and verbal fluency in adults with attention deficit/hyperactivity disorder (ADHD)

Chapter $3 \quad$ Sustained and focused attention deficits in adult attention deficit hyperactivity disorder (ADHD)

Chapter $4 \quad$ Verbal learning and organisational strategies in adults with ADHD: ADHD subtypes and the impact of comorbidity

Chapter 5 Automatic and controlled processes underlying verbal fluency in adults with ADHD: Productivity over time, clustering, and switching

Chapter 6 Effect of stimulant medication on response inhibition and attention in adults with attention deficit hyperactivity disorder

Chapter $7 \quad$ Concluding remarks

Summary

Samenvatting

Dankwoord

Curriculum vitae 

8I Introduction 
Attention Deficit Hyperactivity Disorder (ADHD) is a highly prevalent neurodevelopmental disorder which, at least in childhood and adolescence, is characterised by inattention, hyperactivity, and impulsivity symptoms (American Psychiatric Association [APA], 2000). In order to diagnose an individual child, adolescent or adult with ADHD, some symptoms that cause impairment must be present since before the age of seven years old. Furthermore, according to the Diagnostic and Statistical Manual for Mental Disorders ([DSM-IV]; APA, 1994), symptoms (six or more per cluster) must persist for at least six months such that the behavior is inappropriate for the developmental level. The symptoms must also cause clinical impairment in at least two environmental settings (e.g. school, home, work). During the past decades, there has been increasing evidence for the persistence of ADHD in adulthood. This has resulted in a reconceptualisation of ADHD as a lifespan disorder (APA, 2000; Barkley, 1998; Manuzza, Klein, \& Moulton, 2003; Seidman, 2006). Investigators have suggested that adult ADHD is a valid disorder that can be reliably diagnosed in various countries and cultural backgrounds (Kooij et al., 2004; Murphy \& Schachar, 2000; Seidman, 2006; Spencer, Biederman, Wilens, \& Faraone, 1998). Given that most research has focused on ADHD in children and little research has been conducted on adults with $A D H D$, this dissertation focuses on the underlying nature and expression of behavioral symptoms in adults with ADHD.

\section{Background and epidemiology}

ADHD affects approximately $3 \%$ to $10 \%$ of the school-age population (Barkley, 1998; Kroes et al., 2001; Tannock, 1998). Longitudinal studies have suggested that $30 \%$ to $70 \%$ of the affected children continue to experience symptoms in their adult lives (Biederman et al., 1995; Biederman et al., 1998; Manuzza \& Klein, 2000; Weiss \& Trokenberg-Hechtman, 1993), thereby suggesting that ADHD is present in $2 \%$ to $4.7 \%$ of adults, worldwide (Faraone \& Biederman, 2005; Murphy \& Barkley, 1996). Estimations of the prevalence of ADHD in adults vary considerably and might reflect heterogeneity in this ADHD population, as well as controversy about the strict use of DSM-IV criteria. More specifically, the DSM-IV manual does not provide a separate definition for adult ADHD. Therefore, some clinicians use the threshold originally set for children with ADHD (at least six symptoms out of nine) based on the DSM-IV (APA, 1994), whereas others use modified thresholds (five out of nine criteria for adults), based on the idea that the cut-off point used for children may be too stringent for adults with ADHD (Barkley, 1998; Kooij et al., 2004).

Recent studies revealed that symptoms of hyperactivity and impulsivity decline with increasing age while symptoms of inattention remain a core problem (Biederman, Mick, \& Faraone, 2000; Faraone, Biederman, \& Mick, 2005). In contrast to children with ADHD, gender differences in the prevalence of ADHD 
are less pronounced in adults, with an estimated ratio of 1-2:1 (for men: women) (Biederman et al., 2002). This might reflect an under-diagnosis of women with ADHD. Women, as well as girls, are known to exhibit mainly internalising and attentional problems, whereas men, or boys, are characterised by more externalising problems. As a result, caregivers seek faster for help for these men who in turn tend to be diagnosed quicker than women (Biederman et al., 2002). Furthermore, some symptoms of childhood ADHD (e.g., climbing trees) described in the DSM-IV are inappropriate for adults as these adults display other symptoms (e.g., more inner restlessness) (Barkley, 1998). Clearly, the DSM-IV criteria are not fully applicable for adults with ADHD. Additionally, the pattern of ADHD can change in adulthood. This may contribute to the under-diagnosis of ADHD in adults in current clinical practice (Kooij et al., 2004).

This under-diagnosis is unfortunate, as follow-up studies have demonstrated that these ADHD symptoms in adults impair daily functioning. Adults with ADHD typically have poorer academic outcomes, more occupational difficulties, increased relational problems, more impairment in peer relationships, lower socioeconomic status, lower self-esteem, and more traffic accidents and violations (Faraone et al., 2000). Furthermore, like ADHD in children, the problems and heterogeneity in the adult population are usually intensified by the presence of comorbid disorders (see later in this chapter).

Adult ADHD is a disorder that has a substantial impact on society. Evidently, clear and early assessment of the disorder in adulthood is important in order to tackle current trends of under-diagnosis and to provide effective treatment for these adults. In contrast to the many studies that have focused on school-age children and adolescents, research into the behavioral characteristics of adults with ADHD is relatively scarce and has only recently been given attention in the scientific literature. In particular, the cognitive function profile and underlying mechanisms of adult ADHD has, to date, not been extensively examined. Therefore, the present dissertation aims to increase our knowledge of adult ADHD from a cognitive perspective. Before describing the aims of the present dissertation and the studies that have been conducted, some background information on the topics is presented below.

\section{Neuropsychological research in ADHD adults: A cognitive perspective}

\section{Cognitive deficits}

It has been hypothesised that an underlying brain abnormality is involved in ADHD (Seidman, 2006). More specifically, recent theoretical models of the socalled 'fronto-striatal circuitry dysfunction' and 'attention networks' have emerged in research on children and adults with ADHD (Durston, 2003). Neuro-imaging studies have revealed smaller volumes in parts of the (pre)-frontal cortex, corpus callosum, cerebellum, and basal ganglia structures, as well as less activation of 
these areas and the anterior cingulate in children and adults with ADHD (Bush, Valera, \& Seidman, 2005; Castellanos \& Tannock, 2002; Durston, 2003; Seidman, Valera, \& Makris, 2005). In addition, researchers are taking increasing interest in the measurement of input and output-related information processes through means of cognitive tests to evaluate the hypothesis of underlying brain dysfunction in ADHD.

Although research with school-age children and adolescents with ADHD (Barkley, 1998; Pennington \& Ozonoff, 1996; Seidman, 2006) has enhanced our knowledge on ADHD, far less neuropsychological studies have been performed in adults with ADHD. The cognitive functions that are frequently found to be impaired in adults with ADHD are the more complex higher-order processes, also called the 'executive functions' (EF) (Barkley, 1998; Boonstra, Oosterlaan, Sergeant, \& Buitelaar, 2005; Hervey, Epstein, \& Curry, 2004; Seidman, 2006; Woods, Lovejoy, \& Ball, 2002). The term 'executive function' has been conceptualised as an umbrella term for the interrelated higher-order cognitive processes responsible for goal-directed and contextually appropriate behavior (Alvarez \& Emory, 2006; Anderson, 2002; Lezak, 1995; Pennington \& Ozonoff, 1996; Spreen \& Strauss, 1998). An important and informative model concerning executive functioning is Peter Anderson's (2002) proposed developmental model of EF. In this model, EF is conceptualised as four distinct cognitive domains that operate in an integrative manner as can be seen Figure 1. The first domain, attentional control, includes the capacity to (1) selectively attend to specific stimuli; (2) inhibit prepotent responses; (3) focus attention for a prolonged period; and (4) regulate and monitor actions. Impairments in this domain generally involve impulsive behavior, inappropriate responses, lack of self-control, and failure to complete tasks or correct errors. The second domain, information processing, refers to the fluency, efficiency, and speed of output response. Deficits in this domain manifest as reduced output, delayed responses, and slowed reaction times. The third domain, cognitive flexibility, includes the ability to shift between response sets, to learn from mistakes, to devise alternative strategies, and to divide attention. This domain also includes working memory. Impairments associated with this domain include perseverative behavior, such as continuing to make the same mistakes. The fourth and final domain, goal setting, is regarded as the ability to develop new initiatives and concepts, the capacity to plan actions in advance, and the use of efficient strategies in approaching tasks. Deficits in this domain generally involve poor problem solving, disorganisation, the use of less efficient strategies and poor conceptual reasoning (Anderson, 2002). As can be seen in Figure 1, attentional control processes influence the functioning of the three other executive domains, whereas information processing, goal setting and cognitive flexibility are more inter-related and inter-dependent in that the various cognitive processes in each domain receive and process stimuli from the other various sources. 


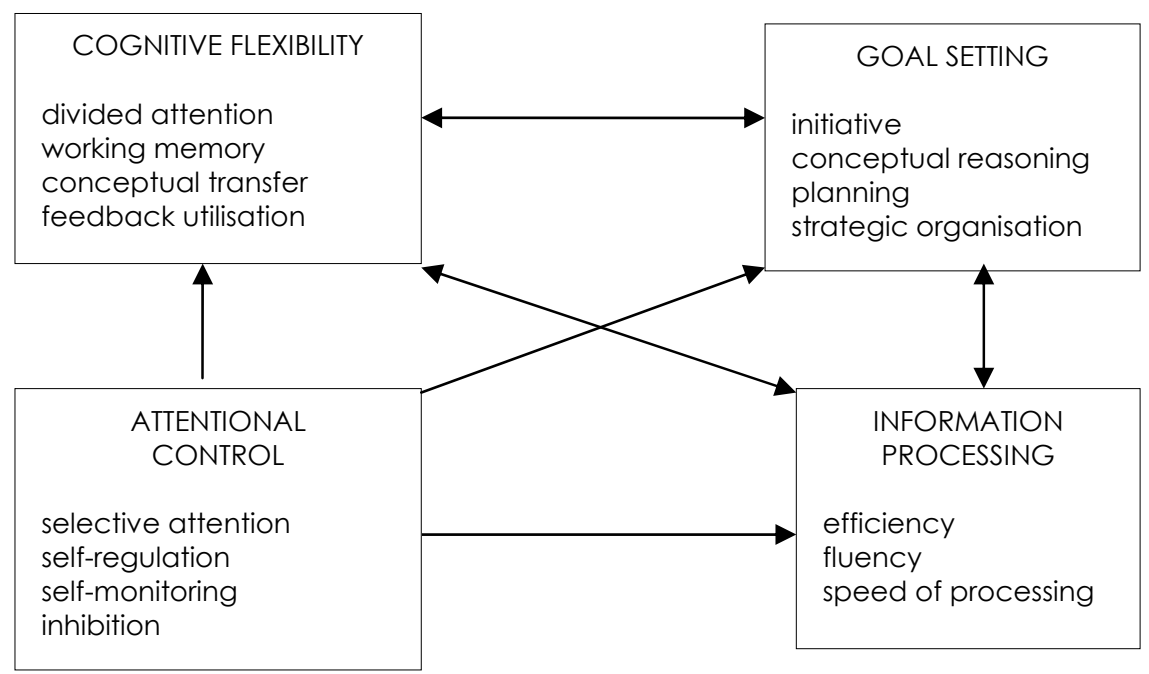

Figure 1. Proposed model of executive function (source: Anderson, P. (2002), Child Neuropsychology, 8, p. 73)

Some neuropsychological studies have reported that, in comparison to healthy control subjects, ADHD adults demonstrate poorer performance in skills belonging to each of these four domains of EF. The topics investigated include 'response inhibition' (Barkley, 1998; Lijfijt, Kenemans, Verbaten, \& van Engeland, 2005), 'verbal fluency', 'working memory', 'planning and organisation', and 'cognitive flexibility' (Boonstra, Oosterlaan, Sergeant, \& Buitelaar, 2005; Hervey, Epstein, \& Curry, 2004; Roth \& Saykin, 2004; Woods, Lovejoy, \& Ball, 2002). These deficits, however, have not been integrated and tested in the model of EF posited by Anderson (2002). Furthermore, the results of the abovementioned studies have been inconsistent and, at times, contradictory. This suggests that the core cognitive deficit underlying ADHD is not yet fully understood. Consequently, this EF model was used as the building block to incorporate the cognitive functions and higher-order processes that were studied in the present dissertation.

Several theoretical models of potential single neuropsychological correlates in ADHD have emerged in recent years (reviewed in Pennington, 2005; Sergeant, Geurts, Huijbregts, Scheres, \& Oosterlaan, 2003). These models can be categorised into two kinds of approaches, namely the cognitive deficit approach and the motivational deficit approach. One of the leading theoretical cognitive models is the 'Inhibition Model' (Barkley, 1997; 1998). According to Barkley's disinhibition theory, behavioral inhibition is regarded as a 'core' deficit in ADHD that, in turn, causes impairments in EF and the regulation of motor output behavior. These include four EFs, namely working memory, self regulation of affect-motivation-arousal, internalisation of speech, and reconstitution of internally represented information (Barkley, 1997, 1998). 
An alternative theory that searches causal explanations of ADHD that do not focus on cognitive limitations but rather motivational processes is the 'Cognitive-Energetic Model of Information Processing' (Sergeant, 2005; Sergeant, Oosterlaan, \& van der Meere, 1999). This model proposes that efficient information processing is determined by the interplay of three levels, namely mechanisms of attention, state factors, and management/ executive function. It suggests that the underlying deficit in ADHD is an energetic disturbance in the regulation of certain 'state' factors, namely activation and effort (Sergeant, 2005; Sergeant, Oosterlaan, \& van der Meere, 1999). These, in turn, cause symptoms of ADHD. However, to date, there is still controversy and no consensus on what the core cognitive deficit of ADHD is and whether a single core deficit can sufficiently explain the symptoms of ADHD (Pennington, 2005).

A recent model, known as the 'Dual Pathway Model,' has been supported by human imaging data, and purports that, in ADHD, separate pathways exist for the cognitive deficits and motivational deficits (Pennington, 2005; Sonuga-Barke, 2005). In this model, cognitive deficits, in particular EF, are related to one frontalstriatal circuit and motivational deficits are linked to another frontal-striatal circuit. Each of these pathways can be deficient in one and the same individual with ADHD (Sonuga-Barke, 2005). The primary focus in the present dissertation is the cognitive pathway, as we endeavored to evaluate the EF model of Anderson (2002) in adults with ADHD. In addition, by examining adults with ADHD and the mechanisms and processes that underlie their poor performance, we will able to yield more insight into their core cognitive impairments. This will be explained in more detail in the following section.

\section{Process approach measures}

Most previous neuropsychological studies on adult ADHD generally investigated common traditional EF outcome measures such as response latency, errors, and total scores. However, it has been suggested that these EF measures result from a web of underlying cognitive processes, such as automatic and controlled processes, response variability, and time duration effects (Sergeant et al., 1999). The experimental approaches and cognitive neuroscience perspectives that use methods derived from information processing theories and tasks offer great potential for unravelling and differentiating the elemental processes involved in higher-order executive dysfunctions in adults with ADHD (Seidman et al., 2006). Process approaches can be used to provide better explanations for the inconsistent findings generated by common measures and traditional tests of executive functioning. These approaches also allow for the examination of the various executive function compounds. In the following paragraphs, we describe some examples of process approaches.

Studies of attention and information processing have demonstrated that overall response times in information processing tasks are typically 'slower' in children with ADHD (Börger et al., 1999; De Sonneville, Njiokiktjen, \& Bos, 1994; De 
Sonneville, Visser, \& Licht, 1999; Kalff et al., 2003; Leth-Steensen, King Elbaz, \& Douglas, 2000; Oosterlaan, Logan, \& Sergeant, 1998). In particular, studies have demonstrated a decrease in response speed (e.g., reaction time) with time on task (TOT) and high positive skewness in children with ADHD (De Sonneville, Njiokiktjen, \& Bos, 1994; De Sonneville, Visser, \& Licht, 1999; Leth-Steensen, King Elbaz, \& Douglas, 2000; Swaab-Barneveld et al., 2000; Van der Meere \& Sergeant, 1988; Van der Meere, Wekking, \& Sergeant, 1991). Investigating TOT performance can provide more insight into the differences between automatic and controlled information processing. Automatic processing is generally fast and relatively unconscious. It requires little attention capacity, whereas controlled processing is slow, effortful and more attention-demanding (Shiffrin \& Schneider, 1977). In children with ADHD, evidence has been found of impairments on different kinds of tasks that require more controlled information processing (Bocherding et al., 1988; A.C. Kalff, 2002; Van der Meere \& Sergeant, 1988), but also on those that elicit automatic processing (Hurks et al., 2004). TOT performance, as well as the underlying automatic and controlled processes in adults with ADHD, needs to be more extensively investigated.

A promising approach that could provide more insight into the previously mentioned underlying automatic and controlled cognitive processes is the investigation of 'executive response organisation strategies'. Research in both children and adults with ADHD is gradually focusing on the use of elaborative executive control strategies that can be elicited by cognitive tasks, such as verbal fluency (Hurks et al., 2004; Mahone, Koth, Cutting, Singer, \& Denckla, 2001; Tucha et al., 2005) or verbal learning and memory tasks (Holdnack et al., 1995; Roth et al., 2004; Seidman, Biederman, Weber, Hatch, \& Faraone, 1998). Further research into the use of these executive strategies will provide more information about whether these strategies are predictors of cognitive performance level and whether these strategies can be used to treat adults with ADHD.

A final common finding is that performance fluctuation from moment to moment is greater in children with ADHD than children without ADHD. Notably, these children have an excessive trial-by trial response variability (e.g. intra-trial variability in each subject) across a variety of speeded reaction time tasks and information processing tasks that measure various aspects of attention and inhibition (e.g. continuous performance tasks, stop-signal tasks) (Börger \& Van der Meere, 2000; Castellanos et al., 2005; Castellanos \& Tannock, 2002; De Sonneville, Njiokiktjen, \& Bos, 1994; Douglas, 1999; Kuntsi, Oosterlaan, \& Stevenson, 2001; Leth-Steensen et al., 2000; Tannock, 2003). Response variability is an important measure of stability of performance or effort allocation, and reflects a high frequency of responses with very short latency and a high frequency of very slow responses (Kuntsi et al., 2001; Leth-Steensen et al., 2000; Tannock, 2003). This pattern of intra-trial variability in subjects instead of variability across the group of subjects has only been limited challenged in adults with ADHD. Some authors reported a higher variability in ADHD adults compared to healthy controls, 
though results remain incongruent (Epstein et al., 1998; Epstein et al., 2001; Hervey et al., 2004; Lijffijt et al., 2005). Further research into this variability in adults with ADHD is necessary, not only because 'attention lapses' remains an important symptom reported in clinical practice, but also because its role as an important marker of (sustained) 'attention' deficit can be adequately addressed.

In summary, the cognitive processes underlying decreased performance on standard summary scores in adults with ADHD have, to date, been inadequately addressed and poorly understood. The abovementioned process approaches provide more insight into the psychological theories of underlying deficits, and the role of control processing in ADHD. Unfortunately, research on process approaches is scarce and those studies that have investigated process approaches have focused primarily on children with ADHD. As a result, the aim of the present dissertation is to provide more insight into these underlying cognitive processes in an adult ADHD population.

\section{Methodological issues}

Aside from the limitations of the previously described use of overall scores, a number of other methodological issues arise when examining ADHD in adults and the associated cognitive processes. Three important issues are discussed in more detail and are addressed in the present dissertation.

\section{Comorbidity and heterogeneity}

The first issue concerns the fact that ADHD adults usually have an elevated rate (varying between $10 \%$ and $70 \%$ ) of comorbid psychiatric conditions, including antisocial and other personality disorders, substance abuse, anxiety and mood disorders, and/or cognitive disorders, such as difficulties in learning and reading (Barkley, Murphy, \& Kwasnik, 1996; Marks, Newcorn, \& Halperin, 2001; Milberger, Biederman, Faraone, Murphy, \& Tsuang, 1995; Millstein, Wilens, Biederman, \& Spencer, 1997; Murphy, Barkley, \& Bush, 2002; Weiss \& Trokenberg-Hechtman, 1993). This high level of comorbidity increases the heterogeneity of symptoms in the adult ADHD population. A debate surrounding neuropsychological research into adults with ADHD pertains to whether the cognitive deficits found in subjects are, in fact, related to ADHD, or are rather an artefact or epiphenomenon of the comorbid disorders (Milberger, Biederman, Faraone, Murphy, \& Tsuang, 1995). A number of competing hypotheses have been proposed to explain non-artefactual comorbidity and are largely based on childhood studies of ADHD and reading disorders (RD). These include the cross-assortment hypothesis, the phenocopy hypothesis, the cognitive subtype hypothesis, and the common aetiology hypothesis (described in Willcutt, Pennington, Olson, Chhabildas, \& Hulslander, 2005). The cross-assortment hypothesis suggests that the comorbidity between ADHD and RD can be best explained by significant cross-assortment mating for 
ADHD and RD that would lead to lower estimates of bivariate heritability. The phenocopy hypothesis, on the other hand, proposes that the co-occurrence of ADHD and RD is due to the primary disorder causing the symptoms of the other disorder in the absence of the cognitive correlates associated with the secondary disorder, thereby suggesting a clean double dissociation between ADHD and RD. The cognitive subtype hypothesis suggests that comorbid ADHD and RD is, in fact, the reflection of a third disorder which has a different pattern of external correlates than would be expected from the additive combination of each disorder separately, thus suggesting that the whole is more than the sum of each part. The last and most commonly adopted hypothesis in regards to children with ADHD and RD is the common aetiology hypothesis which suggests that ADHD and RD share the same aetiological influences and that deficits in the comorbid $A D H D+R D$ exhibit the additive combination of deficits associated with each disorder on its own. However, the influence of comorbidity in adults with ADHD and the comparison of adults with ADHD to these comorbid groups have not been addressed extensively in neuropsychological research. In the present dissertation, the effect of comorbidity on deficits in adults with ADHD and the specificity of deficits were investigated by testing both adults diagnosed with ADHD and one or more comorbid disorders (also called the ADHD + comorbidity group), and a clinical control group of adults who did not have an ADHD diagnosis but had been diagnosed with one or more other psychiatric disorders (also called the non-ADHD pathological control group). These groups were then compared to a pure ADHD without comorbidity group. Our expectations regarding the severity of behavior for each of the clinical and healthy groups are presented in Figure 2. They are primarily based on the common aetiology hypothesis, which suggests that the ADHD with comorbidity group would show more pronounced and combined deficits than the ADHD group or clinical control group alone.

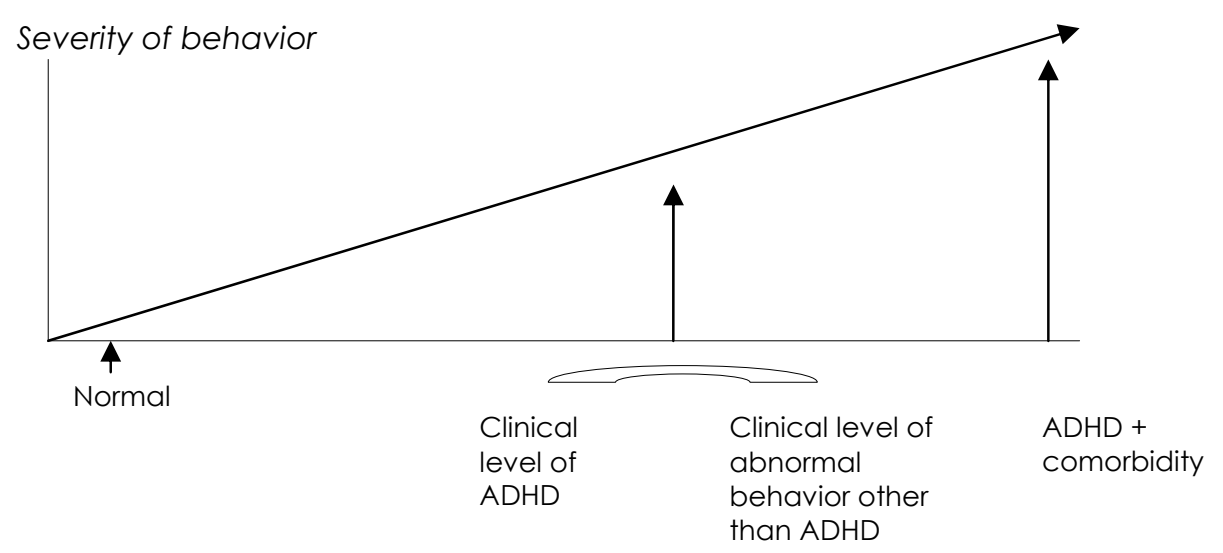

Figure 2. Expectations regarding the severity of behavior from normal behavior to the behavior exhibited by individuals with both ADHD and comorbidity 
Adult ADHD subtypes

The DSM-IV distinguishes three subtypes of ADHD: (1) the predominantly inattentive type, (2) the predominantly hyperactive-impulsive type, and (3) the combined type, which includes symptoms of inattention as well as hyperactivity-impulsivity (APA, 1994). In clinically referred and community based samples, the combined type has been found to be the most prevalent, followed by the predominantly inattentive type, and then the hyperactive-impulsive type which is least prevalent (Barkley, 1998; Kooij et al., 2004; Manuzza, Klein, \& Moulton, 2003; Millstein, Wilens, Biederman, \& Spencer, 1997). Prior studies have suggested that the hyperactive-impulsive subtype resolves into the combined type as age increases, thus leaving only the combined and inattentive subtypes in ADHD adults (Millstein, Wilens, Biederman, \& Spencer, 1997; K. Murphy \& Barkley, 1996; K. R. Murphy, Barkley, \& Bush, 2002). The focus of this present dissertation is, therefore, placed on these two subtypes.

Our second issue relates to the possible differentiation of cognitive strengths and weaknesses in these two subtypes (combined versus inattentive) in adults with ADHD. A number of studies have suggested that the inattentive subtype is more often associated with cognitive sluggish tempo, deficits in selective attention, and processing speed, while the combined subtype has been characterised by deficits in EF, such as inhibition, attention, verbal working memory, and verbal fluency, as well as by the combined deficits of the inattentive and hyperactive-impulsive subtype (Dinn, Robbins, \& Harris, 2001; Gansler et al., 1998; Hervey, Epstein, \& Curry, 2004; Nigg et al., 2005). Despite these findings, the results of research pertaining to ADHD in adults remain varied and scarce. Therefore, investigation of cognitive functioning in terms of subtype in adult ADHD is considered necessary. In the present dissertation, this issue was addressed.

\section{Influence of stimulant medication}

Worldwide, methylphenidate (MPH) is the most commonly prescribed stimulant drug in the treatment of children with ADHD. It yields positive clinical effects on ADHD symptoms in 60 to $70 \%$ of all subjects. Similar positive effects have also been reported in adults with ADHD (Wilens, Spencer, \& Biederman, 2002). Neuropsychological evidence indicates that stimulant drugs (such as methylphenidate) have beneficial effects on cognitive deficits in both children and adults with ADHD (Wilens, Spencer, \& Biederman, 2002). Surprisingly, research on how MPH affects higher-order cognitive processes in adults with ADHD is limited. A better understanding of the cognitive processes affected by MPH is expected to improve our knowledge about theoretical models, and have important implications for the further treatment of adults with ADHD. Therefore, in the present dissertation, the influence of stimulant medication was controlled for in various ways (e.g., included as a covariate, pre- and post treatment trials) depending on the research questions within the studies. 


\section{Aims and outline of the dissertation}

ADHD is a seriously impairing disorder that clearly affects the entire lifespan of the individual, from childhood to adulthood. Based on the information described above, we contend that research on ADHD in adults has revealed some limitations. It is, thus, essential to improve our knowledge of ADHD in adulthood. The central goal of the studies described in this dissertation was to disentangle the EF and related underlying cognitive processes in adults with ADHD. Although many aspects of cognitive functioning can be studied, the functions described in the present dissertation were chosen based on research findings in children with ADHD, and because of their importance to the daily life of adults with ADHD, as reported in clinical practice. New process approach measures are proposed to accurately assess behavioral data obtained from cognitive and informationprocessing tasks. These new insights are, in turn, applied to determine: (i) the impact of comorbidity and, thus, specificity of the cognitive deficits in adults with ADHD; (ii) whether ADHD subtypes can be differentiated from each other in subcomponents of EF and cognitive processes; and (iii) whether methylphenidate influences these cognitive processes in adults with ADHD. In light of these aims, several cross-sectional studies were conducted. Each of these studies focused on one or more distinct cognitive functions in adults diagnosed with ADHD (combined or inattentive subtype). The age range in the studies varied from 18 to 61 years. Adults over the age of 61 were not included because of the known agerelated cognitive deterioration after 60 years and the influence of (pre-) retirement (Houx, \& Jolles, 1993; Houx, Vreeling, \& Jolles 1991; Salthouse, 1996). A short overview of the chapters described in this dissertation is provided below.

Chapter 2: Interference control, working memory, concept shifting and verbal fluency in adults with attention deficit hyperactivity disorder (ADHD)

An important issue that continues to provoke debate is whether adults with ADHD can be characterised in terms of specific EF deficits. This chapter describes a cross-sectional study in which specific domains of EF were investigated in adults with ADHD. The tasks were chosen in such a way that they tapped into several aspects of $E F$, namely interference control, concept shifting, working memory, and verbal fluency, relative to non-EF, such as processing speed. The impact of comorbidity and the specificity of EF deficits in ADHD were studied as well. The sample included adults with relatively pure ADHD, adults with both ADHD and at least one comorbid disorder, clinically-referred non-ADHD adults, and healthy control subjects. All diagnoses made for the ADHD comorbid and clinical non-ADHD groups were included given the high rate of comorbidity in ADHD. The results are described in chapter 2. 
Chapter 3: Sustained and focused attention deficits in adult attention deficit hyperactivity disorder (ADHD)

A related issue concerns the nature of attention deficits and the high intra-individual variability in adults with ADHD. Chapter 3 presents more detailed information on two aspects of attention and underlying information processes by use of a computerised attention test battery. In addition, a simple computerised motor test was used to control for a speed component that may influence task performance in adults with ADHD. This study evaluated: (i) whether focused and sustained attention deficits are present in adults with ADHD, and if so, whether they are specific to ADHD; and (ii) the effect of time on task (TOT). The study also addressed the impact of comorbidity on task and process performance by comparing the results of adults with ADHD and no comorbidity, adults with ADHD and comorbid disorders, clinically-referred non-ADHD adults, and adult healthy control subjects.

\section{Chapter 4: Verbal learning and organisational strategies in adults with ADHD:}

\section{ADHD subtypes and the impact of comorbidity}

Forgetfulness is a frequently reported feature of ADHD in adults. A cognitive function that is highly associated with EF in ADHD is verbal memory. There are no current data available on verbal learning and response organisation for different subtypes of ADHD in adults. Therefore, the objective of this study was to examine more extensively verbal learning, underlying organisational strategies and the role of comorbidity in adults with specific subtypes of ADHD. In addition, the study tested the continuum hypothesis by comparing adults with ADHD-combined subtype with adults with ADHD-inattentive subtype and healthy control adults, using an auditory verbal learning task.

Chapter 5: Automatic and controlled processes underlying verbal fluency in adults with ADHD: productivity over time, clustering, and switching Verbal fluency is an additional EF that is widely used in the clinical assessment of ADHD and research. It is used to evaluate cognitive performance in adults with ADHD. The most common fluency measure is derived by calculating the total number of correct words generated during 60 seconds on fluency tasks. Evidence regarding verbal fluency deficits in adults with ADHD is still inconclusive. A new method for analysing fluency tasks was applied in the study reported in Chapter 5. The pattern of word production over 'time' and 'search strategies', such as clustering and switching, and their relationship to automatic and controlled information processing on two 
types of fluency tasks, were investigated in adults with ADHD-combined subtype.

Chapter 6: Effect of stimulant medication on response inhibition and attention in adults with attention deficit hyperactivity disorder

Currently available theories and neuropsychological data suggest deficits in response inhibition and attention in adults with ADHD, and indicate that stimulant medication improves these cognitive processes. However, research on what core deficit underlies the behavioural symptoms of adult ADHD, and on the efficacy of stimulants on these two cognitive processes is limited. In order to increase our knowledge about these two issues concerning adults with ADHD, two aims were defined in chapter 6. The first aim of the study was to examine whether adults with ADHD (combined subtype) show a primary core deficit in 'response inhibition' or more general deficits in 'attention'. The second aim was to test whether stimulant treatment affects these two processes, as assessed by use of a stop signal task with ADHD adults and healthy control subjects.

\section{Chapter 7: Concluding remarks}

In this final chapter, the findings of the studies are summarised and discussed in the context of this dissertation and recent literature. In addition, methodological considerations and theoretical implications for further research, as well as, recommendations for clinical practice are presented. 


\section{References}

Alvarez, J. A., \& Emory, E. (2006). Executive function and the frontal lobes: A meta-analytic review. Neuropsychology Review, 16(1), 17-42.

American Psychiatric Association (2000). Diagnostic and Statistical Manual of Mental Disorders: DSM-IV-TR. Washington, DC: American Psychiatric Press.

American Psychiatric Association (1994). Diagnostic and statistical manual of mental disorders (4th ed.). Washington DC: American Psychiatric Association.

Anderson, P. (2002). Assessment and development of executive function (EF) during childhood. Child Neuropsychology, 8(2), 71-82.

Barkley, R. A. (1997). Behavioral inhibitions, sustained attention, and executive functions: constructing a unifying theory of ADHD. Psychological Bulletin, 121, 65-94.

Barkley, R. A. (1998). Attention-Deficit/ Hyperactivity Disorder: A handbook for diagnosis and treatment (2nd ed.). New York: The Guilford Press.

Barkley, R. A., Murphy, K., \& Kwasnik, D. (1996). Psychological adjustment and adaptive impairments in young adults with ADHD. Journal of Attention Disorders, 1 (1), 41-54.

Biederman, J., M., E., Faraone, S. V., Braaten, E., Doyle, A., Spencer, T., et al. (2002). Influence of gender on attention deficit hyperactivity disorder in children referred to a psychiatric clinic. American Journal of Psychiatry, 159(36-42).

Biederman, J., Faraone, S. V., Mick, E., Spencer, T., Wilens, T., Kiely, K., et al. (1995). High risk for attention deficit hyperactivity disorder among children of parents with childhood onset of the disorder: a pilot study. American Journal of Psychiatry, 152, 431-435.

Biederman, J., Faraone, S. V., Taylor, A., Sienna, M., Williamson, S., \& Fine, C. (1998). Diagnostic continuity between child and adolescent ADHD: Findings from a longitudinal clinical sample. Journal of the American Academy Child and Adolescence Psychiatry, 37(30), 305-313.

Biederman, J., Mick, E., \& Faraone, S. V. (2000). Age-dependent decline of attention deficit/hyperactivity disorder: Impact of definition of remission. American Journal of Psychiatry, 157, 816-818.

Bocherding, B., Thompson, K., Kruesi, M., Bartko, J., Rapoport, J. L., \& Weingartner, H. (1988). Automatic and effortful processing in attention deficit/hyperactivity disorder. Journal of Abnormal Child Psychology, 16, 333-345.

Boonstra, A. M., Oosterlaan, J., Sergeant, J. A., \& Buitelaar, J. K. (2005). Executive functioning in adult ADHD: a meta-analytic review. Psychological Medicine, 35, 1097-1 108.

Börger, N., \& Van der Meere, J. (2000). Motor control and state regulation in children with ADHD: A cardiac response study. Biological Psychology, 51, 247-267.

Börger, N., Van der Meere, J., Ronner, A., Alberts, E., Geuze, R., \& Bogte, H. (1999). Heart rate variability and sustained attention in ADHD children. Journal of Abnormal Child Psychology, 27(1), 25-33.

Bush, G., Valera, E. M., \& Seidman, L. J. (2005). Functional neuroimaging of attentiondeficit/hyperactivity disorder: A review and suggested future directions. Biological Psychiatry, 57, 1273-1284.

Castellanos, F. X., Sonuga-Barke, E. J. S., Scheres, A., Di Martino, A., C., H., \& Walters, J. R. (2005). Varieties of attention-deficit/hyperactivity disorder-related intra-individual variability. Biological Psychiatry, 57, 1416-1423. 
Castellanos, F. X., \& Tannock, R. (2002). Neuroscience of attention-deficit/hyperactivity disorder: the search for endophenotypes. Nature Reviews Neuroscience, 3, 617-628.

De Sonneville, L. M. J., Njiokiktjen, C., \& Bos, H. (1994). Methylphenidate and information processing: I. Differentiation between responders and nonresponders: II. Efficacy in responders. Journal of Clinical and Experimental Neuropsychology, 16, 877-897.

De Sonneville, L. M. J., Visser, M., \& Licht, R. (1999). Attention and information processing in 4 and 5 year old children: Results of a computerized assessment tech-nique. In B. P. L. M. D. Brinker, P. J. Beek, A. N. Brand, S. J. Maarse \& L. J. M. Mulder (Eds.), Cognitive Ergonomics, clinical assessment and computer-assisted learning: Computers in Psychology. (pp. 204-217). Lisse, Nederland: Swets \& Zeitlinger.

Dinn, W. M., Robbins, N. C., \& Harris, C. L. (2001). Adult Attention-Deficit/Hyperactivity Disorder: Neuropsychological correlates and clinical presentation. Brain and Cognition, 46(1-2), 114-121.

Douglas, V. I. (1999). Cognitive control processes in attention deficit hyperactivity disorder. In H. C. Quay \& A. E. Hogan (Eds.), Handbook of Disruptive Behavior Disorders (pp. 105-138). New York: Kluwer Academic / Plenum Publishers.

Durston, S. (2003). A review of the biological bases of ADHD: What have we learned from imaging studies. Mental Retardation and Developmental Disabilities, 9, 184-195.

Faraone, S. V., \& Biederman, J. (2005). What is the prevalence of adult ADHD? Results of a population screen of 966 adults. Journal of Attention Disorders, 9(2), 384-391.

Faraone, S. V., Biederman, J., \& Mick, E. (2005). The age-dependent decline of attention deficit hyperactivity disorder: A meta-analysis of follow-up studies. Psychological Medicine, 36, 159-165.

Faraone, S. V., Biederman, J., Spencer, T., Wilens, T., Seidman, L. J., Mick, E., et al. (2000). Attention-Deficit/Hyperactivity Disorder in adults: An Overview. Biological Psychiatry, 48, 9-20.

Gansler, D. A., Fucetola, R., Krengel, M., Stetson, S., Zimering, R., \& Makary, C. (1998). Are there cognitive subtypes in adult Attention Deficit/Hyperactivity Disorder? The Journal of Nervous and Mental Disease, 186, 776-781.

Hervey, A. S., Epstein, J. N., \& Curry, J. F. (2004). Neuropsychology of adults with AttentionDeficit/ Hyperactivity Disorder: A meta-analytic review. Neuropsychology, 18(3), 485503.

Holdnack, J. A., Moberg, P. J., Arnold, S. E., Ruben, C., Gur, R. C., \& Gur, R. E. (1995). Speed of processing and verbal learning deficits in adults diagnosed with Attention Deficit Hyperactivity Disorder. Neuropsychiatry, Neuropsychology, and Behavioral Neurology, 8(4), 282-292.

Houx, P. J., \& Jolles, J. (1993). Age-related decline of psychomotor speed: effects of age, brain health, sex, and education. Perceptual and Motor Skills, 76(1), 195-211.

Houx, P. J., Vreeling, F. W., \& Jolles, J. (1991). Age associated cognitive decline is related to biological life-events. In R. C. Mclachlan, B. Winblad \& H. M. Wisniewski (Eds.), Alzheimer's Disease: Basic mechanisms, diagnosis and therapeutic strategies. (pp. 353-359). Chichester: Wiley.

Hurks, P. P. M., Hendriksen, J. G. M., Vles, J. S. H., Kalff, A. C., Feron, F. J. M., Kroes, M., et al. (2004). Verbal fluency over time as a measure of automatic and controlled processing in children with ADHD. Brain and Cognition, 55, 535-544. 
Kalff, A. C. (2002). Neurocognitive performance and demographic variables in children at risk of Attention-Deficit/Hyperactivity Disorder. Maastricht: Neuropsych Publishers.

Kalff, A. C., De Sonnevile, L. M. J., Hurks, P. P. M., Steyaert, J., Zeben, T. M. C. B. v., Vles, J. S. $\mathrm{H}$., et al. (2003). Low- and high-level controlled processing in executive motor control tasks in 5-6-year-old children at risk of ADHD. Journal of Child Psychology and Psychiatry, 44(7), 1049-1057.

Kooij, J. J. S., Buitelaar, J. K., van den Oord, E. J., Furer, J. W., Rijnders, C. A. T., \& Hodiamont, P. P. G. (2004). Internal and external validity of Attention-Deficit Hyperactivity Disorder in a population-based sample of adults. Psychological Medicine, 34, 1-11.

Kroes, M., Kalff, A. C., Kessels, A. G. H., Steyaert, J., Feron, F., Van Someren, A. J. W. G. M., et al. (2001). Child psychiatric diagnoses in a population of Dutch school-children aged 6 to 8 years. Journal of the American Academy of Child and Adolescence Psychiatry, 40(12), 1401-1409.

Kuntsi, J., Oosterlaan, J., \& Stevenson, J. (2001). Psychological mechanisms in hyperactivity: I Response inhibition deficit, working memory impairment, delay aversion, or something else? Journal of Child Psychology and Psychiatry, 42 (2), 199-210.

Leth-Steensen, C., King Elbaz, Z., \& Douglas, V. I. (2000). Mean response times, variability, and skew in the responding of ADHD children: a response time distributional approach. Acta Psychologica, 104, 167-190.

Lezak, M. D. (1995). Neuropsychological assessment (3rd ed.). Oxford: Oxford University Press.

Lijfijt, M., Kenemans, J. L., Verbaten, M. N., \& van Engeland, H. (2005). A meta-analytic review of stopping performance in attention-deficit/hyperactivity disorder: Deficient inhibitory motor control? Journal of Abnormal Psychology, $114(2), 216-222$.

Mahone, E. M., Koth, C. W., Cutting, L., Singer, H. S., \& Denckla, M. B. (2001). Executive function in fluency and recall measures among children with Tourette syndrome or ADHD. Journal of the Internatio-nal Neuropsychological Society, 7, 102-111.

Manuzza, S., \& Klein, R. G. (2000). Long-term prognosis in attention-deficit/ hyperactivity disorder. Child and Adolescent Psychiatric Clinics of North America, 9(3), 711-726.

Manuzza, S., Klein, R. G., \& Moulton, J. L. (2003). Persistence of Attention-Deficit/Hyperactivity Disorder into adulthood: What have we learned from the prospective follow-up studies? Journal of Attention Disorders, 7(2), 93-100.

Marks, D. J., Newcorn, J. H., \& Halperin, J. M. (2001). Comorbidity in Adults with AttentionDeficit/Hyperactivity Disorder. Annals of New York Academy of Sciences, 931, 216238.

Milberger, S., Biederman, J., Faraone, S. V., Murphy, J., \& Tsuang, M. T. (1995). Attention deficit hyperactivity disorder and comorbid disorders: issues of overlapping symptoms. American Journal of Psychiatry, 152, 1793-1799.

Millstein, R. B., Wilens, T. E., Biederman, J., \& Spencer, T. J. (1997). Presenting ADHD symptoms and subtypes in clinically referred adults with ADHD. Journal of Attention Disorders, 2 (3), 159-166.

Murphy, K., \& Barkley, R. A. (1996). Prevalence of DSM-IV symptoms of ADHD in adult licensed drivers: im-plications for clinical diagnosis. Journal of Attention Disorders, 1 , 147-161. 
Murphy, K. R., Barkley, R. A., \& Bush, T. (2002). Young adults with Attention Deficit Hyperactivity Disorder: Subtype differences in comorbidity, educational, and clinical history. The Journal of Nervous and Mental Disease, 190(3), 147-157.

Murphy, P., \& Schachar, R. (2000). Use of self-ratings in the assessment of symptoms of Attention Deficit Hyperactivity Disorder in adults. American Journal of Psychiatry, 157, $1156-1159$.

Nigg, J. T., Stavro, G., Ettenhofer, M., Hambrick, D. Z., Miller, T., \& Henderson, J. M. (2005). Executive functions and ADHD in adults: Evidence for selective effects on ADHD symptom domains. Journal of Abnormal Psychology, $114(3), 706-717$.

Oosterlaan, J., Logan, G. D., \& Sergeant, J. A. (1998). Response inhibition in AD/HD, CD, comorbid $A D / H D$, anxious, and control children: a meta analysis of studies with the stop task. Journal of Child Psychology and Psychiatry, 39, 41 1-425.

Pennington, B. F. (2005). Toward a new neuropsychological model of attention-deficit/ hyperactivity disorder: Subtypes and multiple deficits. Biological Psychiatry, 57, 12211223.

Pennington, B. F., \& Ozonoff, S. (1996). Executive functions and developmental psychopathology. Journal of Child Psychology and Psychiatry, 37, 51-87.

Roth, R. M., \& Saykin, A. J. (2004). Executive dysfunction in attention-deficit/hype-activity disorder: cognitive and neuroimaging findings. Psychiatric Clinics of North America, 27, 83-96.

Roth, R. M., Wishart, H. A., Flashman, L. A., Riordan, H. J., Huey, L., \& Saykin, A. J. (2004). Contribution of organizational strategy to verbal learning and memory in adults with Attention-Deficit/ Hyperactivity Disorder. Neuropsychology, 18(1), 78-84.

Salthouse, T. A. (1996). The processing-speed theory of adult age differences in cognition. Psychological Review, $103(3)$, 403-428.

Seidman, L. J. (2006). Neuropsychological functioning in people with ADHD across the lifespan. Clinical Psychology Review, 26, 466-485.

Seidman, L. J., Biederman, J., Weber, W., Hatch, M., \& Faraone, S. V. (1998). Neuropsychological functioning in adults with attention-deficit hyperactivity disorder. Biological Psychiatry, 44, 260-268.

Seidman, L. J., Valera, E. M., \& Makris, N. (2005). Structural brain imaging of attention deficit/hyperactivity disorder? Biological Psychiatry, 57, 1263-1272.

Sergeant, J. A. (2005). Modeling Attention-Deficit/Hyperactivity Disorder: A critical appraisal of the cognitive-energetical model. Biological Psychiatry, 57, 1248-1255.

Sergeant, J. A., Geurts, H., Huijbregts, S., Scheres, A., \& Oosterlaan, J. (2003). The top and the bottom of ADHD: A neuropsychological perspective. Neuroscience and biobehavioral reviews, 27, 583-592.

Sergeant, J. A., Oosterlaan, J., \& van der Meere, J. (1999). Information processing and energetic factors in attention-deficit/hyperactivity disorder. In H. C. Quay \& A. E. Hogan (Eds.), Handbook of Disruptive Behavior Disorders (pp. 75-104). New York: Kluwer Academic / Plenum Publishers.

Shiffrin, R. M., \& Schneider, W. (1977). Controlled and automatic human information processing: II. Perceptual learning, automatic attending, and a general theory. Psychological Review, 84, 127-190. 
Sonuga-Barke, E. J. S. (2005). Causal models of Attention-Deficit/Hyperactivity Disorder: From common simple deficits to multiple developmental pathways. Biological Psychiatry, 57, 1231-1238.

Spencer, T., Biederman, J., Wilens, T. E., \& Faraone, S. V. (1998). Adults with Attention-Deficit/ Hyperactivity Disorder: A controversial diagnosis. Journal of Clinical Psychiatry, 59([suppl 7]), 59-68.

Spreen, O., \& Strauss, E. (1998). A compendium of neuropsychological tests: Administration, norms, and commentary. (2nd ed.). New York: Oxford University Press.

Swaab-Barneveld, H., De Sonnevile, L. M. J., Cohen-kettenis, P., Gielen, A., Buitelaar, J., \& Van England, H. (2000). Visual sustained attention in a child psychiatric population. Journal of the American Academy of Child and Adolescent Psychiatry, 39(5), 651659.

Tannock, R. (1998). Attention Deficit Hyperactivity Disorder: Advances in cognitive, neurobiological, and genetic research. Journal of the Child Psychology and Psychiatry, 39(1), 65-99.

Tannock, R. (2003). Neuropsychology of attention disorders. In S. J. Segalowitz \& I. Rapin (Eds.), Handbook of Neuropsychology (Vol. 8, pp. 753-784). Amsterdam, the Netherlands: Elsevier Science B.V.

Tucha, O., Mecklinger, L., Laufkötter, R., Kaunzinger, I., Paul, G. M., Klein, H. E., et al. (2005). Clustering and switching on verbal and figural fluency functions in adults with attention deficit hyperactivity disorder. Cognitive Neuropsychiatry, 10(3), 231-248.

Van der Meere, J., \& Sergeant, J. (1988). Controlled processing and vigilance in hyperactivity: Time will tell. Journal of Abnormal Child Psychology, 16(6), 641-655.

Van der Meere, J., Wekking, E., \& Sergeant, J. A. (1991). Sustained attention and pervasive hyperactivity. Journal of Child Psychology and Allied Disciplines, 32, 275-584.

Weiss, G., \& Trokenberg-Hechtman, L. (1993). Hyperactive children grown up. New York: The Guilford Press.

Wilens, T. E., Spencer, T. J., \& Biederman, J. (2002). A review of pharmacotherapy of adults with attention-deficit/hyperactivity disorder. Journal of Attention Disorders, 5, 189202.

Willcutt, E. G., Pennington, B. F., Olson, R. K., Chhabildas, N., \& Hulslander, J. (2005). Neuropsychological analyses of comorbidity between reading disability and attention deficit hyperactivity disorder: In search of the common deficit. Developmental Neuropsychology, 27(1), 35-78.

Woods, S. P., Lovejoy, D. W., \& Ball, J. D. (2002). Neuropsychological characteristics of adults with ADHD: A comprehensive review of initial studies. The Clinical Neuropsychologist, $16(1), 12-34$. 



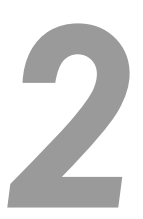

\section{Interference control, working memory, concept shifting and verbal fluency in adults with attention deficit/hyperactivity disorder (ADHD)}




\section{Abstract}

This study aimed to examine four domains of executive functioning in adults with ADHD, namely interference control, concept shifting, verbal fluency, and verbal working memory. Four groups of participants were included: (i) adults diagnosed with ADHD ( $n=20$ ) [ADHD]; (ii) adults diagnosed with both ADHD and one or more comorbid disorder(s) $(n=22)\left[\mathrm{ADHD}^{+}\right]$; (iii) adults referred for ADHD due to ADHD symptomatology but not diagnosed as such ( $n=34)$ [non-ADHD]; and (iv) healthy controls $(n=136)$. ADHD-related deficits (independent of comorbidity) were revealed for concept shifting and verbal working memory. In addition, the $\mathrm{ADHD}^{+}$and non-ADHD groups displayed deficits in terms of general processing speed. Given that these deficits were not found in the ADHD-group, we contend that these deficits are likely attributable to comorbidity rather than ADHD itself. Contrary to our expectations, these findings do not correspond with the cognitive subtype hypothesis. 


\section{Introduction}

Attention Deficit/Hyperactivity Disorder (ADHD) is marked by a triad of core symptoms, namely inattention, hyperactivity and impulsivity (American Psychiatric Association [APA], 1994). Although ADHD has historically been regarded as a childhood disorder, evidence indicates that ADHD persists into adulthood for 50$80 \%$ of all individuals ever diagnosed with ADHD (Biederman et al., 1995; Biederman et al., 1998; Manuzza \& Klein, 2000; Weiss \& Trokenberg-Hechtman, 1993). Clearly, ADHD can and does have a significant impact on many individuals in adulthood. These individuals often experience problems with organization, discipline and setting priorities. Additionally, they are often accident prone and have difficulty maintaining a stable job or marriage (Barkley, Murphy, \& Kwasnik, 1996; Faraone et al., 2000).

An involvement of prefrontal regions and other interconnected subcortical structures (e.g., the cerebellum and caudate nucleus), underlying an executive dysfunction, has been found in both ADHD children and adults (Biederman \& Faraone, 2002; Bush et al., 1999; Durston, 2003; Rubia et al., 1999; Seidman, Valera, \& Makris, 2005; Vaidya et al., 1998). Researchers are taking an increasingly greater interest in the measurement of input and output-related information processes to evaluate the hypothesis of an underlying brain dysfunction in ADHD. The primary focus is on tests measuring deficits in executive functioning (Barkley, 1998). The term EF is a multidimensional construct that refers to a set of higherorder cognitive functions necessary for goal-directed and contextually appropriate behavior (Lezak, 1995; Spreen \& Strauss, 1998). Cognitive operations related to EF include the following: initiation, planning, hypothesis generation, cognitive flexibility, decision making, regulation, judgment, feedback utilization, self perception, response inhibition, interference control, working memory, planning, cognitive flexibility, and fluency (Denckla, 1996; Pennington \& Ozonoff, 1996). Although the hypothesized EF deficits have been explored extensively in children, to date, little research has been conducted on EF deficits in adults with ADHD and the studies that have been conducted have generated inconsistent results (Dinn, Robbins, \& Harris, 2001; Gansler et al., 1998; Hervey, Epstein, \& Curry, 2004; Holdnack et al., 1995; Johnson et al., 2001; Lovejoy et al., 1999; Murphy, 2002; Murphy, Barkley, \& Bush, 2001; Rapport, Van Voorhis, Tzelepis, \& Friedman, 2001; Sergeant, Geurts, \& Oosterlaan, 2002; Seidman, Biederman, Weber, Hatch, \& Faraone, 1998; Seidman et al., 2004; Walker, Shores, Trollor, Lee, \& Sachdev, 2000; Woods, Lovejoy, \& Ball, 2002). Some studies have found that adults with ADHD, when compared to healthy control subjects, perform poorer on interference control (i.e., using the Stroop Color-Word Test) (Dinn et al., 2001; Murphy et al., 2001; Rapport et al., 2001), mental flexibility (i.e., the Trail Making Test) (Gansler et a., 1998; Holdnack et al., 1995; Lovejoy et al., 1999), and semantic verbal fluency (Lovejoy et al., 1999; Walker et al., 2000). At the same time, other studies have demonstrated no differences between adults with ADHD and healthy control 
subjects for the Trail Making Test (Walker et al., 2000) and verbal fluency (Barkley et al., 1996; Johnson et al., 2001). Because of this lack of clarity, we endeavored, in our study, to evaluate differences in a subset of executive functions in four groups of adults, namely adults with ADHD, adults with comorbid ADHD, adults with non-diagnosed ADHD and healthy control subjects.

When examining EF in ADHD, and when considering the inconsistent results of previous studies, a number of issues must be considered. Firstly, we must be aware that the EF domain is non-unitary and that performance measures are only modestly correlated (Nigg, Blaskey, Huang-Pollock, \& Rappley, 2002). EF is a broad domain and the specification of the components in this domain must be carefully considered (Barkley, 1997; Pennington \& Ozonoff, 1996). Unfortunately, the precise specification of subdomains is still being debated. Therefore, for the purposes of the current study, we included a limited number of EF domain components. These were chosen in accordance with Pennington and Ozonoff (1996) classification system and included: (i) interference control, which is defined as protection from self-directed responses that result from disruption by competing events and responses; (ii) concept shifting, which is the ability to regulate one's own behavior or to shift a course of thought or action according to the demands of the situation; (iii) verbal fluency, which is the ability to generate spontaneous verbal production and organization while relying on rule-governed strategies for retrieval of verbal information under time constraints; and (iv) verbal working memory, which is a buffer in which a limited amount of information can be actively retained over a relatively short period of time.

The second issue that must be considered when examining EF and ADHD is that earlier studies have suggested that EF tasks may also tap into non-EF tasks such as attention capacity and information processing speed (Nigg et al., 2002; Pennington \& Ozonoff, 1996; Sergeant et al., 2002). In order to determine whether poor performance on an EF task is due to either working memory, verbal fluency, concept shifting or interference control, it is important to include control tasks that measure non-EF functions (Sergeant et al., 2002). Consequently, the study presented here included assessments that would control for non-EF functions. More specifically, we used an assessment of output response speed, a factor found to be impaired in individuals with ADHD (Nigg et al., 2002), as a control for non-EF demands and for its relevance to associated functions, such as effort.

A third issue that needs to be considered is that ADHD in adults often cooccurs with other psychiatric conditions, including antisocial and other personality disorders, substance abuse, anxiety, and mood disorders and learning disabilities. The prevalence of comorbid disorders is not incidental. Comorbidity occurs in $30 \%$ to $60 \%$ of all adults with ADHD (Milberger, Biederman, Faraone, Murphy, \& Tsuang, 1995; Millstein, Wilens, Biederman, \& Spencer, 1997; Murphy, Barkley, \& Bush, 2002). Many of the comorbid disorders associated with ADHD are known to directly affect the cognitive abilities of the individual (Pennington \& Ozonoff, 1996; Seidman et al., 1997). Therefore, it is difficult to determine if cog- 
nitive deficits are attributable to ADHD or to the presence of the comorbid disorder or to both. Only a few studies have examined the extent to which EF deficits are actually a function of ADHD by controlling for comorbidity (i.e., Murphy et al., 2001; Seidman et al., 1998; Walker et al., 2000). This is unfortunate as failing to control for the impact of comorbid symptoms weakens the validity of neuropsychological investigations in clinical settings (Walker et al., 2000). Therefore, in the present study, we controlled comorbidity in two ways. Firstly, we employed a subgroup comparison in which a group of adults with comorbidity $\left(\mathrm{ADHD}^{+}\right)$were compared to a group of adults without comorbidity (ADHD-). According to the cognitive subtype hypothesis (Rucklidge \& Tannock, 2002), the co-occurrence of ADHD together with another syndrome generates a third disorder that is due, at least in part, to etiological factors that are distinct from those that increase suseptibility to both disorders alone. This hypothesis thus predicts that the comorbid group will exhibit a different pattern of external correlates than would be expeced based on the additive combination of the correlates of each disorder when they occur separately. This hypothesis corresponds with the findings reported by Oosterlaan, Logan, and Sergeant (1998). These authors demonstrated that chilren with ADHD and comorbid ODD/OCD had enhanced EF deficits when comaed to children with only ADHD or only ODD/CD. Unfortunately, studies investigaing this hypothesis that focus on adults remain inconclusive. The present study investigates this hypothesis in relation to adults in detail. The second means by which we controlled for comorbidity was done by including a clinically-referred control group (non-ADHD). This group was comprised of individuals that had been referred for potential diagnosis because they exhibited ADHD symptoms but, upon assessment, did not meet the diagnostic criteria for ADHD. Members of this group may display psychopathology other than ADHD or no psychopathology at all. Nonetheless, the inclusion of this group as a control group was consideed interesting for the determination of differences in neurocognitive dysfunction given the fact that this group had previously been referred for the clinical assessent of ADHD symptomatology.

In summary, the primary goal of the present study was to investigate the performance of adults with and without ADHD on four executive domains, namely interference control, concept shifting, working memory and verbal fluency, and on one non-EF domain, namely processing speed. Our second goal was to determine the impact of comorbidity and the specificity of EF deficits in adults with ADHD. This was done by comparing adults with relatively pure ADHD (ADHD) to adults with both ADHD and at least one comorbid disorder (ADHD ${ }^{+}$) and also to clinically-referred non-ADHD adults. Based on previous studies, we first hypothesized that participants with ADHD would show impairments on all four EF domains. We also hypothesized that, if the ADHD groups with and without comorbidity performed differently than the healthy control group, this difference would be attributable to the presence of ADHD. Further, we hypothesized that, if the $\mathrm{ADHD}^{+}$group and non-ADHD group performed differently than the ADHD- group 
and/or healthy controls this difference would be attributable to the presence of comorbidity in both groups. If only the $\mathrm{ADHD}^{+}$group would perform differently than the healthy control or non-ADHD groups, this would be attributable to ADHD and/or comorbidity differences in the groups. We also expected that the ADHD+ group would show more pronounced and more specific deficits when compared to the ADHD- group and the non-ADHD group. This expectation was based on the cognitive subtype hypothesis, which posits that the impact of comorbid disorders is more than the sum of each separate disorder (Rucklidge \& Tannock, 2002). In selecting our sample, we only included young adults aged 20 to 41 years given the existence of age-related changes in neurocognitive function for middle aged subjects (Houx, Jolles, \& Vreeling, 1993; Houx, Vreeling, \& Jolles, 1991; Salthouse, 1996).

\section{Method}

\section{Participants}

Participants in the ADHD-, ADHD', and non-ADHD groups were clinically-referred by a medical specialist to the outpatient facility for adults with ADHD at Vijverdal Psychiatric Hospital in Maastricht, the Netherlands. The participants were consecutive referrals recruited over a period of five years from 1998 to 2003 . At the time of referral, all individuals exhibited one or more ADHD symptoms and sought the help of a medical specialist who then referred them to this specialized outpatient clinic. Participant characteristics of all groups are presented in Table 1. A multidisciplinary diagnostic assessment, that corresponds with procedures used in other studies (i.e., Barkley et al., 2001; Barkley, Murphy, DuPaul, \& Bush, 2002; Walker et al., 2000), was applied at Vijverdal. The diagnostic assessment consisted of standardized psychiatric and (neuro)psychological diagnostic and research protocols. This included a semi-structured diagnostic interview and a clinical interview with both the patient and a cross-informant (usually a parent or a spouse), both of which were conducted by experienced psychiatrists or health care psychologists. In addition, self-reported rating scales were completed by the patient, both at home and at the diagnostic center. Furthermore, a neuropsychological examination, including well-established tests measuring intelligence and attention and the experimental tasks described in this paper was conducted. Lastly, a review of school reports (if available) was done. The diagnosis was reached by a multidisciplinary ADHD team of board certificated psychiatrists and licensed health care psychologists/clinical neuropsychologists. The diagnosis was determined by clinical consensus ratings of all the available data from the interviews (including medical and psychosocial history taking) and the neuropsychological assessment. All individuals in the ADHD- group received a diagnosis based on the DSM-IV diagnostic criteria of ADHD (APA, 1994) by referring to both current symptoms and retrospective symptoms of childhood ADHD (ADHD com- 
bined type: $n=15$; ADHD predominantly inattentive type: $n=5)$. Individuals included in the ADHD- group displayed no comorbidity. All participants included in the $\mathrm{ADHD}^{+}$group (ADHD combined type: $n=17$; ADHD predominantly inattentive type: $n=5$ ) met the criteria for both ADHD and at least one other DSM-IV classification (see Table 2 for an overview of comorbid disorders). The non-ADHD group was comprised of individuals who, although having been referred for assessment because they displayed ADHD symptoms, did not meet the diagnostic criteria for the disorder $(n=34)$. A high percentage of the adults in this group had at least one DSM-IV classification other than ADHD (see Table 2 for group characteristics regarding DSM-IV diagnoses).

The healthy control group (matched with ADHD and non-ADHD participants for gender, age and IQ; $n=136$ ) was derived from a large, cross-sectional and longitudinal study, called the Maastricht Aging Study (MAAS) ( $N=1823$ ) (Jolles, Houx, Van Boxtel, \& Ponds, 1995, Van Boxtel et al., 1998). These MAAS participants can be regarded as historical controls (Campbell \& Stanley, 1963). The procedure for recruitment of the healthy control subjects in the MAAS study has been described in detail elsewhere (Jolles et al., 1995; Van Boxtel et al., 1998). In short, participants in the MAAS study were randomly drawn from a Registration Network of Family Practices, which is a sample frame for research in health care practice (Metsemakers, Höppener, Knottnerus, Kocken, \& Limonard, 1992). Individuals were invited to participate in the study by their general practitioners, rather than by the project staff, because this was expected to generate greater participation and study compliance. They were also screened by this practitioner for psychosocial contraindications to participation, such as actual major life events. After agreeing to participate, a postal survey questionnaire was sent to the subjects. At the same time, the participants were asked if they were also willing to participate in the additional test program. Healthy participants were selected from the same region, namely the Southern region of the Netherlands, as the clinical participants. Additionally, the health control subjects in the MAAS study were between 24 to 81 years of age at the time of the study. For the purpose of this study only healthy control subjects in the same age range as the ADHD adults were included.

Exclusion criteria for all four groups included: (1) serious head trauma, incluing organic syndromes like meningitis; (2) the presence of neurological pathology or medical disease(s), such as epilepsy, dementia, cerebrovascular disease, parinsonism, malignancies related to the nervous system, and diabetes mellitus; and (3) an IQ score less than 85. ADHD and non-ADHD participants taking medication other than stimulants (i.e., antidepressants) were not excluded. All clinical particiants were asked to discontinue their stimulant medication treatment 24 hours prior to the testing if this was the case as this can influence their task performance (Hervey et al., 2004). Additional exclusion criteria for the healthy control sample were: (1) psychopathology, including ADHD and learning disabilities and other psychiatric disorders; (2) current chronic psychotropic drug use, such as 
medication, alcohol, and drugs as these also co-occur in ADHD adults and these substances can influence task performance. None of the healthy control subjects were taking medication at the time of the study. In total, 212 adults were included in the study (ADHD+, $n=22$; ADHD', $n=20$; non-ADHD, $n=34$; healthy control subjects, $n=136)$.

Table 1. Participant characteristics

\begin{tabular}{lcccccc}
\hline Variable & $\begin{array}{c}\text { ADHD } \\
(n=20)\end{array}$ & $\begin{array}{c}\text { ADHD }^{+} \\
(n=22)\end{array}$ & $\begin{array}{c}\text { Non- } \\
\text { ADHD } \\
(n=34)\end{array}$ & $\begin{array}{c}\text { Controls } \\
(n=136)\end{array}$ & $F$ & $X^{2}$ \\
\hline $\begin{array}{l}\text { Gender (\% } \\
\text { women) }\end{array}$ & 25.0 & 27.3 & 47.1 & 38.2 & & 3.676 \\
$\begin{array}{l}\text { Medication (\% } \\
\text { yes) }\end{array}$ & 15.0 & 40.9 & 38.2 & 0 & & $59.165^{* * *}$ \\
Age $(M, S D)^{b}$ & 29.95 & 31.34 & 28.81 & 30.13 & 1.162 & \\
& $(5.22)$ & $(5.32)$ & $(5.92)$ & $(4.79)$ & & \\
IQ $(M, S D)$ & 103.55 & 108.36 & 102.59 & 105.22 & 1.306 & \\
\hline
\end{tabular}

Note. ADHD = Attention Deficit/Hyperactivity Disorder; ADHD- = ADHD without comorbidity: $\mathrm{ADHD}^{+}=\mathrm{ADHD}$ with comorbidity. a Pearson chi-square test. $b$ The age range is 20 to 41 years at time of the neuropsychological investigation. ${ }^{* * *} p<.001$.

\section{Procedures}

All ADHD, $\mathrm{ADHD}^{+}$, and non-ADHD participants were individually tested. Each completed a comprehensive clinical neuropsychological evaluation at Vijverdal Psychiatric Hospital Maastricht sometime between 1998 and 2003. The cognitive tests selected for the present study were part of an extensive neuropsychological test battery that included attention, planning, other memory tests as well as psychological questionnaires. This battery of tests was administered to all participants in the same sequence. The neuropsychological investigation and collection of data were conducted under the supervision of several experienced and licensed health care psychologists/neuropsychologists. The procedure for all healthy participants in the MAAS study, including the subgroup selected for this study, consisted of a baseline test program (from 1993 till 1995) using the neuropsychological tests described in this paper and an additional questionnaire. The data for the healthy control subjects were collected by test assistants that had been extensively trained in test administration by neuropsychologists and a physician that was also part of the project staff. All participants provided informed consent for participation in the study in written form. 
Table 2. Distribution of psychopathology other than ADHD in the clinicallyreferred groups

\begin{tabular}{lcc}
\hline DSM-IV disorders & ADHD+ & non-ADHD \\
\hline no DSM-disorder & 4 & 7 \\
mood disorder & 3 & 1 \\
anxiety disorder & 8 & 8 \\
PD & 3 & \\
substance-related disorders & & 1 \\
communication disorder & & 1 \\
$\begin{array}{l}\text { adaptation disorder } \\
\text { mood disorder \& anxiety disorder }\end{array}$ & 1 & 1 \\
mood disorder \& PD & & 5 \\
PD \& substance-related disorder & & 1 \\
$\begin{array}{l}\text { PD \& PTSD } \\
\text { PD \& eating disorder }\end{array}$ & & 1 \\
substance-related disorders \& PTSD & & 1 \\
mood disorder \& PD \& substance-related disorders & 1 & 1 \\
PD \& substance-related disorder \& eating disorder \\
$\begin{array}{l}\text { mood disorder \& PD \& substance-related disorders \& } \\
\text { panic disorder with agoraphobia in remission } \\
\text { mood disorder \& PD \& PTSD \& psychosis NOS } \\
\text { mood disorder \& PD \& anxiety disorder \& PTSD \& } \\
\text { bulimia nervosa }\end{array}$
\end{tabular}

Note. $\mathrm{PD}$ = personality disorder, PTSD = post-traumatic stress disorder.

\section{Measures of EF and non-EF}

We selected the following cognitive tests to represent a subset of four major EF domains, namely interference control, mental flexibility, verbal fluency, and verbal working memory (Pennington \& Ozonoff, 1996). To measure non-EF demands reflected by information processing speed and attention, the first components of the Stroop Test and the Concept Shifting Test were utilized. These components of the EF battery and tests are not exhaustive but were chosen because they are sensitive to prefrontal lobe damage (Lezak, 1995) and given that prefrontal dysfunctions are also associated with ADHD (Durston, 2003), we considered these tests most appropriate for our study.

\section{Interference control}

The Stroop Color-Word Test (SCWT). The SCWT involves three subtests that include a hundred stimuli each in a $10 \times 10$ matrix (Stroop, 1935; Hammes, 1973). Participants were asked to read words (Card I) or call out names of the colors represented by colored patches (Card II) as fast as possible. Subsequently, during Card III, participants were asked to name the color of the ink in which a 
color name is printed. Performance on Card III is largely determined by the time taken to discard irrelevant but very salient information (i.e., verbal reading) in favor of a less obvious aspect (i.e., color naming). The SCWT is a measure of general information processing speed (reading), (selective) attention, inhibition, and interference susceptibility (Houx et al., 1993; Lezak, 1995; Stroop, 1935). The dependent variable was the averaged total time to complete Card I and Card II (SCWT_12 = [Card I + Card II]/2) as a non-EF control measure of information processing speed. The total time required to complete Card III (SCWT_3) and the interference score (SCWT interference = SCWT_3 - SCWT_12) were used as measures of interference control that proved to be valid in previous studies (Valentijn et al., 2005; Van der Elst, Van Boxtel, Van Breukelen, \& Jolles, 2006a).

\section{Mental flexibility}

The Concept Shifting Test (CST). The CST is a modified version of the Trial Making Test, which is a subtest of the Halstead-Reitan Battery (Reitan, 1958) and is often used to measure information processing speed, (divided) attention, simple motor speed, visual conceptual and visuomotor tracking, and mental flexibility (Houx \& Jolles, 1993; Houx et al., 1991; Jolles et al., 1995; Vink \& Jolles, 1985). During the administration of Card A and B, the participant was asked to cancel out consecutively numbered circles on a worksheet (Card A) and then the same number of consecutively lettered circles (Card B). In Card C, the participant was asked to cancel out the same number of consecutively numbered and lettered circles on another worksheet by alternating between the two sequences (i.e., 1 - A, 2 - B). The dependent measure was the averaged total time to complete Card $A$ and $B$ (CST_AB $=[$ Card $A+$ Card $B] / 2)$ as a non-EF control measure of informa-tion processing speed. Total time to complete Card $C$ (CST_C) and the CST shifting score (CST shifting = CST_C - CST_AB) were used as a measure of mental flexibility (Valentijn et al., 2005; Van der Elst, Van Boxtel, Van Breukelen, \& Jolles, in press).

\section{Verbal Fluency}

Verbal Fluency Test (VFT). The Verbal Fluency Test is a subtask of the Groningen Intelligence Test (GIT; Luteijn \& van der Ploeg, 1983). In the VFT, participants were asked to name as many words as they could that related to a predefined semantic category (i.e., animals, professions) for one full minute. Standardized C-scores correcting for age (range 0 - 10) were calculated for each category. The average of these C-scores (fluency average) was used as a dependent measure. This has been regarded as a measure of semantic category verbal fluency and strategy-driven retrieval from semantic memory (Jolles et al., 1995; Lezak, 1995; Van der Elst, Van Boxtel, Van Breukelen, \& Jolles, 2006b).

\section{Verbal working memory}

The Auditory Verbal Learning Task (AVLT). In this test, 15 frequently used monosyllabic words were presented, one after another, in a fixed order at a rate 
of one every two seconds (Brand \& Jolles, 1985; Jolles et al., 1995; Van der Elst, Van Boxtel, Van Breukelen, \& Jolles, 2005). After the presentation of all words, participants were asked to reproduce as many words as possible (immediate recall). This procedure was repeated five times. Twenty minutes after the fifth recall, participants were asked again to recall as many words as possible (delayed recall). Word production on the first trial (AVLT Trial 1) was included as a measure of immediate memory span or working memory based on previous literature (Van der Elst et al., 2005; Schmidt, 1996; Spreen \& Strauss, 1998).

\section{Intelligence}

Estimate of IQ: Doing Sums, Mental Rotation, \& Analogies. The subtask 'doing sums' required the participant to, within a time period of one minute, correctly complete as many adding sums as possible. The subtask 'mental rotation' required participants to indicate which two-dimensional shape needed to be selected from a larger set to completely fill a given space. The third subtask 'analogies' can be regarded as a multiple choice version of the well-known 'Similarities' subtest of the WAIS-R (Wechsler, 1981), in which participants are asked to indicate which of the five alternatives is similarly related to a given word as the two words in the example. These three subtasks are part of the Groningen Intelligence Test, which is a frequently used Dutch intelligence test (Luteijn \& van der Ploeg, 1983). The combination of these subtasks is believed to be a good approximation of a full-scale IQ (Luteijn \& van der Ploeg, 1983). The mean score was 100 (SD = 15). The GIT is comparable to the WAIS-R as evidenced by a correlation between the GIT- IQ and the WAIS-IQ that ranges from .72 to .91 (Luteijn \& van der Ploeg, 1983).

\section{Data analysis}

Firstly, all dependent variables were examined for extreme values (i.e., scores $>3$ times the interquartile length from the median). With respect to the specificity of the analyses, it is important to note that the number of par-ticipants excluded differed for each dependent variable as follows: SCWT_3 ( $n=2$ non-ADHD participants), SCWT interference ( $n=1$ non-ADHD partici-pant and $n=1$ healthy control), CST_C \& CST shifting ( $n=1$ healthy control). In addition, incomplete test results were found for the SCWT ( $n=2$ ADHD $^{+}, n=1$ non-ADHD), CST $(n=4$ $\mathrm{ADHD}^{+}, n=1$ ADHD), the VFT $\left(n=3 \mathrm{ADHD}^{+}\right)$and the AVLT $\left(n=1 \mathrm{ADHD}^{+}\right)$. These data were believed to be missing at random. Missing data occurred because of technical problems during testing or because parts of the investigation that had already taken place in another setting with the participant were not reassessed. No non-compliance occurred. Adults with extreme scores or missing data were excluded pair-wise from the analyses. Therefore, the degrees of freedom in the $F$ statistics varied according to the number of participants that had completed the task under study and/or the number of extreme values deleted. 
Furthermore, bivariate correlational analyses between the outcome measures and demographic variables (i.e., IQ and medication) were performed in order to determine if these variables should be included as covariates in the analyses (see Table 3). Only low associations were found between the executive outcome measures and IQ and medication usage (see Table 3). Therefore, the demographic measures were not included as covariates.

Separate General Linear Model (GLM) univariate analyses of variance were used per task with correction for unequal sample sizes. $P$ values of .05 or lower were considered statistically significant. If the omnibus $F$ test was significant, we conducted post-hoc pairwise comparisons with Bonferroni corrections. The Cohen's partial eta squared $\left(\eta_{p}^{2}\right)$ is reported for all group comparisons to convey effect size for GLM univariate analyses. This is done in accordance with recommendations found in academic literature (Pierce, Block, \& Aguinis, 2004), whereby the values of partial eta squared are considered equal to eta squared values (and $r$ squared) in the case of GLM univariate analyses. These correspond approximately to the following effect size conventions: small $(0.01)$, medium (0.06), and large (0.14) (Cohen, 1988).

All outcome measures were also screened for violations of assumptions associated with GLM univariate analyses. Levene's test of equality of error variances was significant for SCWT_12 ( $p<.001)$, SCWT_3 $(p<.001)$, SCWT interference $(p<.001)$, CST_C $(p<.001)$, CST shifting $(p<.001)$, and therefore additional nonparametric Kruskal-Wallis tests were performed for the SCWT and CST outcome measures. All statistical analyses were perfor-med using the SPSS package for Windows 11.0 .

\section{Results}

Means and standard errors (SE) for each outcome measure according to group are displayed in Table 4. With regard to the SCWT, the following results were found: Significant group differences were found on averaged total time to complete Card I and Card II (SCWT_12): ADHD+ participants $(p<.001)$ and non-ADHD adults $(p=.019)$ performed slower than the healthy control subjects. Additionally, a significant main effect of group was found on the Stroop Card III (SCWT_3): $\mathrm{ADHD}^{+}$adults were slower than the healthy control subjects $(p=.027)$. On the SCWT interference score, this main effect on SCWT_3 attenuated and thereby no significant group differences were found for this measure. Notably, nonparametric tests were indicated with violations of the assumptions of GLM univariate analyses. However, given that the non-parametric Kruskal-Wallis tests revealed comparable results (with the exception of the absence of significant main effect on SCWT_3 ( $p>$.05), the parametric tests are reported here and in Table 4.

Furthermore, no significant group differences were found on the averaged total time to complete Card A and B (CST_AB). However, significant group diffe- 
rences were found on the CST Card C (CST_C) and the CST shifting score. On the CST_C, we found that adults with $\mathrm{ADHD}^{+}$had significantly lower scores than the healthy control subjects $(p=.015)$. Additionally, the ADHD- group was significantly slower than the non-ADHD group $(p=.044)$ and the healthy control subjects ( $p$ $=.001)$. Post-hoc analyses on the CST shifting score revealed results highly comparable to the CST_C. Non-parametric tests for CST_C and CST shifting revealed results that were comparable to the GLM univariate tests and therefore the parametrics are reported (Table 4).

With regard to the scores on the verbal fluency test (VFT), the results indicate that the non-ADHD group performed worse than the healthy control subjects $(p=.030)$. No other group differences were found. With respect to the AVLT Trial 1, significant group differences in performance were found. Both the ADHDand $\mathrm{ADHD}^{+}$groups had significantly lower scores on this first trial when compared to the healthy control subjects $\left(\mathrm{ADHD}^{+}\right.$vs. healthy control subjects, $p=.048$; ADHD- vs. healthy control subjects, $p=.003)$.

Table 3. Correlations between EF variables, non-EF variables, medication and IQ for the four groups ( $N=212)$

\begin{tabular}{|c|c|c|c|c|c|c|c|c|c|c|}
\hline Variable & 1 & 2 & 3 & 4 & 5 & 6 & 7 & 8 & 9 & 10 \\
\hline 1.SCWT_3 & - & $.93^{* *}$ & $.41^{* *}$ & $.21^{* *}$ & $-.35^{* *}$ & $-.24^{* *}$ & $.68^{* *}$ & $.50^{* *}$ & .09 & $-.25^{* *}$ \\
\hline $\begin{array}{l}\text { 2. SCWT } \\
\text { interference }\end{array}$ & & - & $.31^{* *}$ & $.14^{*}$ & $-.26^{* *}$ & $-.18^{* *}$ & $.38^{* *}$ & $.41^{* *}$ & .04 & $-.22^{* *}$ \\
\hline 3. CST_C & & & - & $.87^{* *}$ & $-.31^{* *}$ & $-.27^{* *}$ & $.41^{* *}$ & $.64^{* *}$ & $.18^{* *}$ & $-.33^{* *}$ \\
\hline 4. CST shifting & & & & - & $-.22^{* *}$ & $-.21^{* *}$ & $.25^{* *}$ & $.20^{* *}$ & .11 & $-.22^{* *}$ \\
\hline 5. Fluency & & & & & - & $.22^{* *}$ & $-.35 * *$ & $-.28^{* *}$ & -.03 & $.27^{* *}$ \\
\hline 6. AVLT Trial 1 & & & & & & - & $-.26^{* *}$ & $-.23^{* *}$ & $-.19 * *$ & $.24^{* *}$ \\
\hline 7. SCWT_12 & & & & & & & - & $.43^{* *}$ & $.16^{*}$ & $-.21^{* *}$ \\
\hline 8.CST_AB & & & & & & & & - & $.18^{* *}$ & $-.33^{* *}$ \\
\hline 9.Medication & & & & & & & & & - & -.05 \\
\hline 10. IQ & & & & & & & & & & - \\
\hline
\end{tabular}

Note. SCWT = Stroop Color-Word Test; CST = Concept Shifting Test; AVLT = Auditory Verbal Learning Task. ${ }^{* *} p<.01$.

In general and as depicted in Table 3, the correlations between the tasks across the four EF domains were low thus suggesting that the EF measures were relatively independent of one another. Correlations between tasks within an EF domain (i.e., SCWT and CST) were high, suggesting that they were measuring the same domain. Correlations between the EF measure and non-EF measures were also low. The only exceptions were a) a high positive correlation between SCWT_3 and both non-EF output speed measures (SCWT_12 and CST-AB); and b) a high positive correlation between CST_C and the non-EF CST_AB measure. 


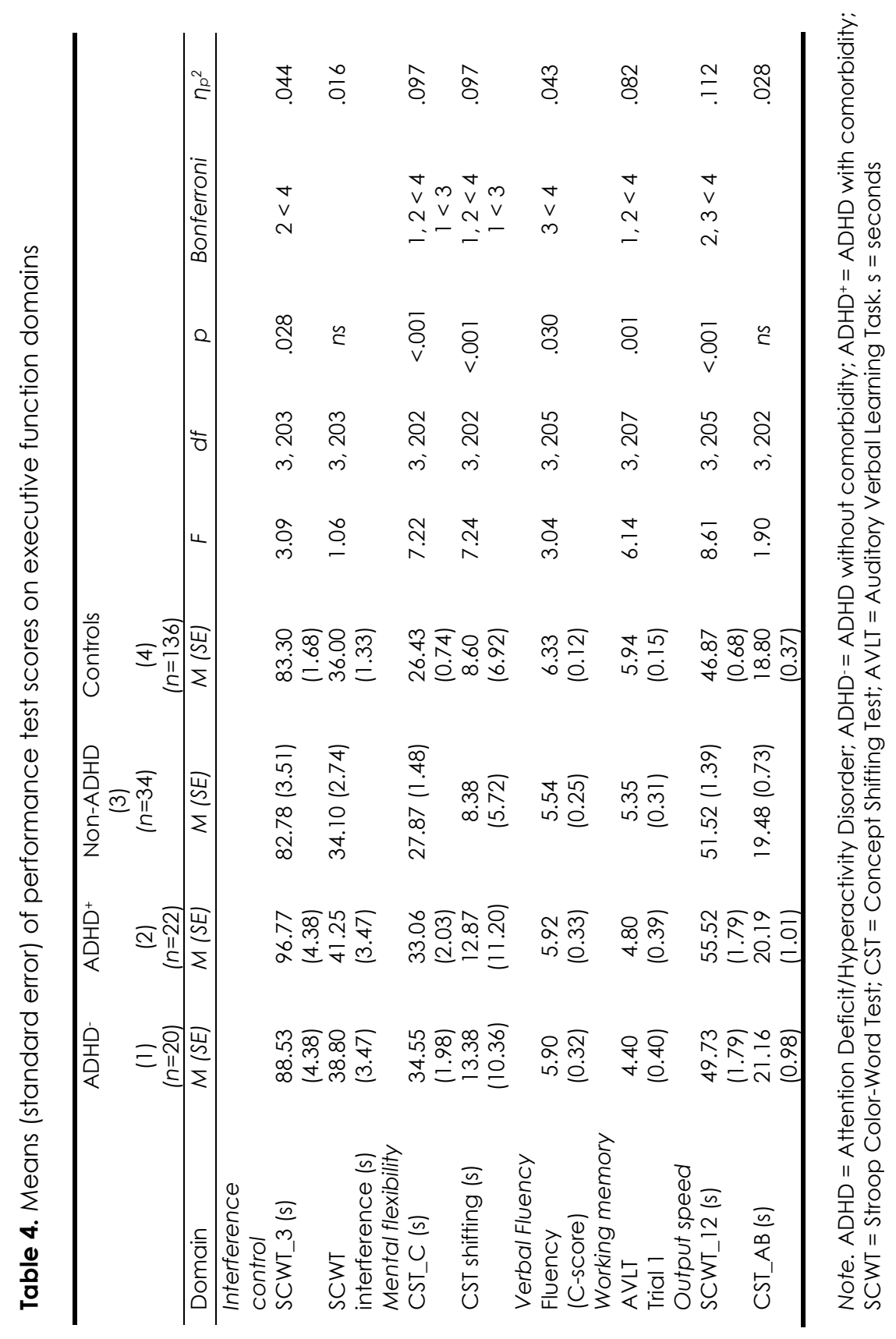




\section{Discussion}

The aims of the study reported were to examine (a) the relationship between adult ADHD and four domains of EF; and (b) the impact of comorbidity on this relationship. This was done in an effort to determine whether ADHD does indeed generate deficits on all four EF domains as suggested by Barkley's model (1997). Therefore, test performance of two ADHD groups (ADHD+ and ADHD-) and two control groups (non-ADHD and healthy control subjects) was contrasted.

Based on the pair-wise comparisons, we found observable ADHD-related deficits (i.e., verbal working memory deficits and deficits in concept shifting) that are independent of comorbidity. The finding that adults with ADHD have problems with verbal working memory measured by the AVLT Trial 1 corresponds with previously reported studies (Holdnack et al., 1995; Lovejoy et al., 1999; Murphy et al., 2001; Nigg, 2001; Walker et al., 2000). Difficulties with verbal working memory have also been reported in clinical practice as forgetfulness in daily activities (APA, 1994). Additionally, the medium effect size on a measure of working memory found in our study is consistent with what has been found in a previous meta-analytic study (Hervey et al., 2004). In addition, this finding that ADHD adults performed worse on immediate recall of AVLT Trial 1 than healthy controls may reflect a disturbance in the verbal storage component demands of Baddeley's model of working memory (1986). Indeed, previous work has demonstrated that deficits on immediate recall of verbal word list learning tasks can be best interpreted by a deficient phonological loop (see review of Hervey et al., 2004). This storage system of the working memory model (Baddeley, 1986; Repovs and Baddeley, 2006) is involved in holding verbal information online and has a limited capacity. Our finding supports this hypothesis. Evidently, this finding has important clinical implications. Adults with ADHD may benefit from working memory training programs and this should be investigated further. It is possible that these group differences may be attributable to an inefficient use of strategies for processing new verbally-unrelated information. This inefficient strategy use has previously been demonstrated in patients with frontal-lobe lesions (Gershberg \& Shimamura, 1995; Stone, Gabrieli, Stebbins, \& Sullivan, 1998). Further research on underlying verbal learning strategies and learning processes in ADHD adults is necessary to confirm our findings.

It is also important to note that, in our study, concept shifting deficits were also specifically related to both ADHD groups. This finding corresponds with previous review studies conducted with adults diagnosed with ADHD (Hervey et al., 2004; Woods et al., 2002). These studies demonstrated that, when compared to healthy control subjects, adults with ADHD performed worse on the TMT-B task, which is a task that requires advanced planning and mental flexibility. Also, in correspondence with earlier studies (Hervey et al., 2004), our study also found a medium effect size on this task. Additionally, our finding that adults with ADHD, irrespective of their comorbidity, have more difficulties with the self regulation of 
their behavior and with shifting from one concept to another supports not only recent theoretical models on $A D H D$ but also the contention that these deficits may be uniquely related to ADHD (Barkley, 1998; Nigg, 2001). However, caution is still needed in this conclusion on specific ADHD deficits as the potential role of comorbidity in this ADHD with comorbidity group cannot be fully ruled out. These problems with shifting from one concept to another and with self control have also been reported in clinical practice as complaints relating to the failure to organize and complete activities (APA, 1994). Similarly to our conclusions on verbal working memory, we contend that the difficulties adults with ADHD experience in regulating their behavior and shifting from one concept to another may be attributable to the use of an inefficient strategy to complete these tasks.

In contrast to our findings on working memory and concept shifting, no ADHD-related effects were found for interference control or verbal fluency. Although similar findings have been reported by other investigators (Corbett \& Stanczak, 1999; Downey, Stelson, Pomerleau, \& Giordani, 1997; Gansler et al., 1998; Johnson et al., 2001; Walker et al., 2000), these results do contradict the findings of other studies described earlier. For example, Barkley's $(1997,1998)$ frequently cited disinhibition model states that deficits in response inhibition, such as interference control, are the core deficits found in individuals with ADHD and that these deficits in response inhibition lead secondarily to general executive function impairments, such as verbal fluency deficits. Unfortunately, most models and studies relating to EF impairments and ADHD have been conducted or are applied to children with ADHD. However, Hervey et al. (2004) did conduct a metaanalytic study with adults and found that, when compared to healthy control subjects, adults with ADHD performed more poorly on verbal fluency tests. Additionally, and in congruence with our study, these authors demonstrated low effect sizes for the Stroop interference. It is possible that the severity of executive dysfunction declines as individuals with ADHD age because they learn to compensate for these deficits in everyday life and thus also in neuropsychological testing settings. Examples of compensatory strategies include expressing hyperactivity though inner restlessness rather that outward displays of hyperactivity, avoiding situations that cause problems or make symptoms more apparent, using work schedules to organize, and delegating boring or complex tasks to partners or significant others (Amstrong, Hayes, \& Martin, 2001).

Furthermore, we found poor performance on verbal fluency in the nonADHD group in comparison to healthy controls. Such a verbal fluency deficit has also been found in individuals with different kinds of psychiatric conditions (such as personality disorders, mood and anxiety disorders, and substance-related disorders) that were also diagnosed in the majority of our non-ADHD participants (Den Hartog, 2002; Fossati, Guillaume, Ergis \& Allilaire, 2003; Moritz et al., 2002; Pope, 2001; Wilens et al., 2003). Our finding of no deficits in verbal fluency in adults with ADHD is in line with two previous studies (Barkley et al., 1998; Johnson et al., 2001). However, the results remain mixed about this issue in adult ADHD 
(see Woods et al., 2002). Future research on quantitative analyses of word production over time and res-ponse organization strategies (e.g., clustering, switching) would be promising in detecting mechanisms underlying verbal fluency performance in adult ADHD. In addition, our analyses showed no significant group differences between the non-ADHD group and the ADHD groups, suggesting that these groups were rather comparable in their performance. This is consistent with an earlier study by Walker et al. (2000). These authors found no significant differences between an ADHD group and a psychiatric group with mood and anxiety disorder on measures of verbal fluency. Inspection of the scores also suggests that there was 'no clinically significant effect' found for the non-ADHD participants as their score fell within the normal/average range.

With respect to our non-EF task demands, including information processing speed, automation of skills and/or (selective) attention, our study demonstrated deficits on SCWT_12 for both the ADHD+ group and the non-ADHD group. Since these deficits occurred only in these two groups and not in the ADHD- group, we contend that these deficits are likely attributable to the presence of comorbidity rather than to ADHD. Evidently, this finding does not correspond with the cognitive subtype hypothesis (Rucklidge \& Tannock, 2002). Strikingly, the findings of our study suggest that effects due to the presence of ADHD and one or more comorbid disorders result from the additive combination of the correlates of each disorder when they occur separately (Willcutt, Pennington, Olson, Chhabildas, \& Hulslander, 2005). Therefore, the non-significance of the interference control measure in ADHD adults may stem from the slower reading of words and listing of colors, which are variables that are also taken into account in the interference control measures. Indeed, we did observe medium effects on the SCWT_12 in our study which corresponds with Hervey and colleagues (2004) results in which small effects on these Stroop measures were found. In contrast to the findings produced by Hervey et al., we found that, in comparison to healthy control subjects, our ADHD participants did not experience more problems with both verbal and fine motor processing, as measured by the CST_AB.

An additional aspect of our study worthy of mention is the associations between tasks across the four EF domains of and between tasks within each EF domain. Our results indicate that the correlations between tasks across the four domains were low and that the correlations between tasks within a given domain (i.e., SCWT and CST) were high. This suggest that the four EF domains where independent of one another and thereby supports our contention that the different tasks measured different underlying EF constructs. We also found low correlations between the majority of the EF and non-EF measures. High correlations between the SCWT_3 and both non-EF output speed measures (SCWT_12 and CST_AB) and between CST_C and the non-EF CST_AB measure were the only exeptions and these were expected given that the non-EF measures were also involved in the SCWT_3 and CST_C tasks. Our finding supports the idea put forth by Sergeant et al. (2002) that the Stroop task and the Concept Shifting task mea- 
sures are rather non-unitary constructs that cannot easily be distinguished from one another. We therefore endeavored to control for these non-EF measures in order to examine the isolated EF deficits and, indeed, when controlling for these measures, no deficits appeared on the interference control measure in adults with ADHD. Clearly, this suggests that different EF and non-EF processes are involved in the Stroop task.

In line with this, we contend that our results can be explained by a general processing speed load problem in both the ADHD and non-ADHD clinical groups. Many of the tasks, including the SCWT and CST, were highly dependent on speed of processing. We therefore argue that lower load of processing speed was confined to ADHD and the clinical comorbidity groups. Indeed, simple processing speed difficulties have also been found in mood disorders (Den Hartog, 2002), which were part of our clinical non-ADHD group. In addition, problems with higher demands on processing speed load were more specifically confined to adult ADHD. Likewise, in pediatric ADHD, processing speed has been suggested as a potential common deficit in comorbidity between ADHD and reading disorder (Willcutt et al., 2005). Our results suggest that processing speed may also be a worthwhile focus for future research on the neuropsychological correlates of adult ADHD and comorbid disorders

\section{Limitations and future research}

The findings reported here need to be interpreted in light of some limitations. Firstly, ADHD is a heterogeneous condition with different subtypes (APA, 1994) and a high rate of comorbidity (Milberger et al., 1995; Millstein et al., 1997; Murphy et al., 2002). Like many other studies on ADHD (see Hervey et al., 2004), the heterogeneity across and within the ADHD sample posed a potential problem in the study presented here. The ADHD+ group was very heterogeneous and this may limit the degree to which we were able to properly interpret the results of the study. This was unavoidable given that, to date, the exact effects of the various comorbid conditions on neuropsychological performance remain unknown and inconsistent. This is exemplified by the following: In a study conducted by Murphy et al. (2001), the authors addressed the effect of comorbidity and concluded that the presence of additional psychopathology (i.e., depressive, anxious or oppositional symptoms) did not affect neuropsychological performance. However, in the meta-analytic study by Hervey et al. (2004), the authors found that adults diagnosed with both ADHD and comorbid disorder displayed greater neuropsychological deficits than individuals with only ADHD and no comoribidity. While the above mentioned studies contradict each other, our study supports aspects of both. Nonetheless, a limitation that remains is our failure to control for the range of comorbid disorders and our treatment of the group as a whole. We therefore are unable to draw any definitive conclusions about the impact of an isolated or additive effect of one or more comorbid disorders in the adults with ADHD. Nonetheless, given that the primary focus of our study was 
placed on determining deficits in adults with ADHD and only secondarily on the effect of comorbidity, we consider our decision to control for comorbidity by comparing a pure ADHD group to both a group with comorbid ADHD and a clinical non-ADHD group to be sufficient.

An additional limitation of our study relates to ADHD subtype differences. We were unable to make comparisons between ADHD subtypes because of the small ADHD subtype sample sizes. Additionally, subtype comparisons were not performed because they were likely to decrease the power of our study or generate Type I and II errors. Nonetheless, we hypothesize, based on the findings of earlier studies (Barkley, 1997, 1998; Nigg et al., 2002), that had the subtypes been compared, EF deficits would have only been found in the adults with the ADHD combined subtype and not in adults with the ADHD inattentive subtype. However, in a recent study by Murphy et al. (2001), no EF differences between different adult ADHD subtypes were found. Moreover, further research exploring subtype differences on tasks with different load on processing speed may shed more light on the differential correlates and mechanisms involved in ADHD disorders.

A further limitation of our study was that, due to our sample size, we examined only four of the many functions included in the domain EF. We were therefore unable to provide a complete breakdown of intact and impaired executive functions in adults with ADHD. However, we were able to examine the aspects of EF most commonly reported as impaired aspects in children with ADHD and also the impact of comorbidity on these cognitive functions in order to determine the degree to which findings with children are consistent and generalize to adults with ADHD.

A last point of concern involves our approach employed to assess our EF and non-EF components. The subtraction approach used to indicate EF components (e.g., Stroop interference control) remains controversial and other measures, such as the proportion score, have been suggested to be a more sophisticated measure (Stuss, Floden, Alexander, Levine, \& Katz, 2001). However, our subtraction approaches for the SCWT as well as the CST are frequently reported in other studies and have proven to be valid measures of EF components (Meijer, 2006; Valentijn et al., 2005; Van Boxtel et al., 1998; Van der Elst et al., 2006a; Van der Elst et al., in press; van Hooren, 2004). In addition, exploratory analyses of the proportion of Stroop and CST interference scores, based on the paper by Stuss et al. (2001), revealed comparable results. Thereby, we found it justified to implement our subtraction approach.

We recommend that future research investigate the usefulness of a larger test battery that includes a wider range of EF and non-EF domains. We also recommend the analyses of more qualitative outcomes, such as number of errors, self corrections, and time segments in verbal fluency. Lastly, we recommend a longitudinal assessment of EF domains to determine the stability of the impairments in the clinical groups. By doing this, a complete breakdown of intact and 
impaired executive function in adults with ADHD with and without comorbidity can be delineated and according to ADHD subtype.

\section{Conclusions}

In conclusion, the current study has demonstrated specific deficits in two domains of executive functioning, namely verbal working memory and concept shifting, for adults with pure ADHD (ADHD) and for adults characterized by ADHD and comorbidity $\left(A D H D^{+}\right)$. No ADHD-related effects were found for interference control or verbal fluency. Our study has also demonstrated that, in contrast to the group of adults with pure ADHD, the group with both ADHD and a comorbid disorder and the group termed non-ADHD displayed processing speed problems. The results thus suggest that these deficits are due to the effect of comorbidity rather than ADHD. The results reported here do not fully correspond with Barkley (1997) who, in ADHD children found a more general weakness in executive function or all EF domains. Our findings do however support the hypothesis that general EF weaknesses are neither necessary nor sufficient to cause all cases of ADHD (Willcutt, Doyle, Nigg, Faraone, \& Pennington, 2005).

\section{Acknowledgement}

The authors wish to thank Jurgen Cornelis and Ben Hirsch, members of the ADHD multidisciplinary team, for their clinical expertise and cooperation. 


\section{References}

American Psychiatric Association (1994). Diagnostic and Statistical Manual of Mental Disorders (4th ed.). Washington DC: American Psychiatric Association.

Amstrong, C. L., Hayes, K. M., \& Martin, R. (2001). Neurocognitive problems in Attention Deficit Disorder. Alternative concepts and evidence for impairment in inhibition of selective attention. Annals of New York Academy of Sciences, 931, 196-215.

Baddeley, A. (1986). Working memory. Oxford, England: Clarendon Press.

Barkley, R. A. (1997). Behavioral inhibitions, sustained attention, and executive functions: constructing a unifying theory of ADHD. Psychological Bulletin, 121, 65-94.

Barkley, R. A. (1998). Attention-Deficit/ Hyperactivity Disorder: A handbook for diagnosis and treatment (2nd ed.). New York: The Guilford Press.

Barkley, R. A., Murphy, K., \& Kwasnik, D. (1996). Psychological adjustment and adaptive impairments in young adults with ADHD. Journal of Attention Disorders, 1 (1), 41-54.

Barkley, R. A., Murphy, K. R., \& Bush, T. (2001). Time perception and reproduction in young adults with Attention Deficit Hyperactivity Disorder. Neuropsychology, 15(3), 351-360.

Barkley, R. A., Murphy, K. R., DuPaul, G. J., \& Bush, T. (2002). Driving in young adults with atten-tion deficit hyperactivity disorder: Knowlegde, performance, adverse outcomes, and the role of executive functioning. Journal of the International Neuropsycholo-gical Society, 8, 655-672.

Biederman, J., \& Faraone, S. V. (2002). Current concepts on the neurobiology of AttentionDeficit/Hyperactivity Disorder. Journal of Attention Disorders, 6, S7-S17.

Biederman, J., Faraone, S. V., Mick, E., Spencer, T., Wilens, T., Kiely, K., et al. (1995). High risk for attention deficit hyperactivity disorder among children of parents with childhood onset of the disorder: a pilot study. American Journal of Psychiatry, 152, 431-435.

Biederman, J., Faraone, S. V., Taylor, A., Sienna, M., Williamson, S., \& Fine, C. (1998). Diagnostic continuity between child and adolescent ADHD: Findings from a longitudinal clinical sample. Journal of the American Academy Child and Adolescence Psychiatry, 37(30), 305-313.

Biederman, J., Mick, E., \& Faraone, S. V. (2000). Age-dependant decline of attention deficit/hyperactivity disorder: Impact of definition of remission. American Journal of Psychiatry, 157, 816-818.

Brand, N., \& Jolles, J. (1985). Learning and retrieval rate of words presented auditory and visually. Journal of General Psychology, 112, 201-210.

Bush, G., Frazier, J. A., Rauch, S. L., Seidman, L. J., Whalen, P. J., Jenike, M. A., et al. (1999). Anterior cingulate cortex dysfunction in attention deficit/hyperactivity disorder revealed by fMRI and the counting group. Biological Psychiatry, 45(12), 1542-1552.

Campbell, D., \& Stanley, J. (1963). Experimental and quasi-experimental designs for research. Boston: Hougton Mifflin.

Cohen, J. (1988). Statistical power analysis for the behavioral sciences. (2nd ed.). Hillsdale, New Jersey: Erlbaum.

Corbett, B., \& Stanczak, D. E. (1999). Neuropsychological performance of adults evidencing attention-deficit hyperactivity disorder. Archives of Clinical Neurops-chology, 14, 373-387.

Den Hartog, M. (2002). Cognitive performance in depression: Patterns and determinants. Unpublished doctoral dissertation, Maastricht University, the Netherlands. 
Denckla, M. B. (1996). A theory and model of executive function: A neuropsychological perspective. In G. R. Lyon \& N. A. Krasnegor (Eds.), Attention, memory, and executive function. (pp. 263-277). Baltimore, M.D.: Paul H. Brooks.

Dinn, W. M., Robbins, N. C., \& Harris, C. L. (2001). Adult Attention-Deficit/Hyperactivity Disorder: Neuropsychological correlates and clinical presentation. Brain and Cognition, 46(1-2), 114-121.

Downey, K. K., Stelson, F. W., Pomerleau, O. F., \& Giordani, B. (1997). Adult Attention Deficit Hyperactivity Disorder: Psychological test profiles in a clinical population. Journal of the Nervous and Mental Disorders, 185, 32-38.

Durston, S. (2003). A review of the biological bases of ADHD: What have we learned from imaging studies. Mental Retardation and Developmental Disabilities, 9, 184-195.

Faraone, S. V., Biederman, J., Spencer, T., Wilens, T., Seidman, L. J., Mick, E., et al. (2000). Attention-Deficit/Hyperactivity Disorder in adults: An Overview. Biological Psychiatry, 48, 9-20.

Fossati, P., Guillaume, L. B., Ergis, A.-M., \& Allilaire, J.-F. (2003). Qualitative analysis of verbal fluency in depression. Psychiatry Research, 117, 17-24.

Gansler, D. A., Fucetola, R., Krengel, M., Stetson, S., Zimering, R., \& Makary, C. (1998). Are there cognitive subtypes in Adult Attention Deficit/Hyperactivity Disorder? The Journal of Nervous and Mental Disease, 186, 776-781.

Gershberg, F. B., \& Shimamura, A. P. (1995). Impaired use of organizational strategies in free recall following frontal lobe damage. Neuropsychologia, 13, 1305-1333.

Hammes, J. (1973). De Stroop Kleur-Woord Test: Handleiding [The Stroop Color-Word Test: Manual]. Amsterdam: Swets \& Zeitlinger.

Hervey, A. S., Epstein, J. N., \& Curry, J. F. (2004). Neuropsychology of adults with AttentionDeficit/Hyperactivity Disorder: A meta-analytic review. Neuropsy-chology, 18(3), 485503.

Holdnack, J. A., Moberg, P. J., Arnold, S. E., Ruben, C., Gur, R. C., \& Gur, R. E. (1995). Speed of processing and verbal learning deficits in adults diagnosed with Attention Deficit Hyperactivity Disorder. Neuropsychiatry, Neuropsychology, and Behavioral Neurology, 8(4), 282-292.

Houx, P. J., \& Jolles, J. (1993). Age-related decline of psychomotor speed: effects of age, brain health, sex, and education. Perceptual and Motor Skills, 76(1), 195-211.

Houx, P. J., Jolles, J., \& Vreeling, F. W. (1993). Stroop interference: aging effects assessed with the Stroop Color-Word Test. Experimental Aging Research, 19(3), 209-224.

Houx, P. J., Vreeling, F. W., \& Jolles, J. (1991). Age associated cognitive decline is related to biological life-events. In R. C. Mclachlan, B. Winblad \& H. M. Wisniewski (Eds.), Alzheimer's Disease: Basic mechanisms, diagnosis and therapeutic strategies. (pp. 353-359). Chichester: Wiley.

Johnson, D. E., Epstein, J. N., Waid, L. R., Latham, P. K., Voronin, K. E., \& Anton, R. F. (2001). Neuropsychological performance deficits in adults with attention deficit/hyperactivity disorder. Archives of Clinical Neuropsychology, 16, 587-604.

Jolles, J., Houx, P. J., Van Boxtel, M. P. J., \& Ponds, R. W. H. M. (1995). The Maastricht Aging Study: Determinants of cognitive aging. Maastricht: Neuropsych Publishers.

Lezak, M. D. (1995). Neuropsychological assessment (3rd ed.). Oxford: Oxford University Press. 
Lovejoy, D. W., Ball, J. D., Keats, M., Stutts, M. L., Spain, E. H., Janda, L., et al. (1999). Neuropsychological performance of adults with attention deficit hyperactivity disorder (ADHD): Diagnostic classification estimates for measures of frontal lobe / executive functioning. Journal of the International Neuropsychological Society, 5, 222-233.

Luteijn, F., \& Ploeg, F. A. E. van der (1983). Handleiding Groninger Intelligentietest (GIT) [Manual Groningen Intelligence Test]. Lisse, The Netherlands: Swets \& Zeitlinger.

Manuzza, S., \& Klein, R. G. (2000). Long-term prognosis in attention-deficit/ hyperactivity disorder. Child and Adolescent Psychiatric Clinics of North America, 9(3), 71 1-726.

Meijer, W. (2006). Cognitive aging: Effects of education and task demands. Unpublished Dissertation, Maastricht University, Maastricht, the Netherlands.

Metsemakers, J. F. M., Höppener, P., Knottnerus, J. A., Kocken, R. J. J., \& Limonard, C. B. G. (1992). Computerized health information in the Netherlands: A registration network of family practices. British Journal of General Practice, 42, 102-106.

Milberger, S., Biederman, J., Faraone, S. V., Murphy, J., \& Tsuang, M. T. (1995). Attention deficit hyperactivity disorder and comorbid disorders: Issues of overlapping symptoms. American Journal of Psychiatry, 152, 1793-1799.

Millstein, R. B., Wilens, T. E., Biederman, J., \& Spencer, T. J. (1997). Presenting ADHD symptoms and subtypes in clinically referred adults with ADHD. Journal of Attention Disorders, 2 (3), 159-166.

Moritz, S., Birkner, C., Kloss, M., Jahn, H., Hand, I., Haasen, C., et al. (2002). Executive functioning in obsessive-compulsive disorder, unipolar depression, and schizophrenia. Archives of Clinical Neuropsychology, 17, 477-483.

Murphy, P. (2002). Cognitive functioning in adults with Attention-Deficit/Hyperactivity Disorder. Journal of Attention Disorders, 5(4), 203-209.

Murphy, K., Barkley, R. A., \& Bush, T. (2001). Executive functioning and olfactory identification in young adults with Attention Deficit-Hyperactivity Disorder. Neuropsychology, $15,211-220$.

Murphy, K. R., Barkley, R. A., \& Bush, T. (2002). Young adults with Attention Deficit Hyperactivity Disorder: Subtype differences in comorbidity, educational, and clinical history. The Journal of Nervous and Mental Disease, 190(3), 147-157.

Nigg, J. T. (2001). Is ADHD a Disinhibitory Disorder? Psychological Bulletin, 127(5), 571-598.

Nigg, J. T., Blaskey, L. G., Huang-Pollock, C. L., \& Rappley, M. D. (2002). Neuropsychological executive functions and DSM-IV ADHD subtypes. Journal of the American Academy of Child and Adolescence Psychiatry, 41 (1), 59-66.

Oosterlaan, J., Logan, G. D., \& Sergeant, J. A. (1998). Response inhibition in AD/HD, CD, Comorbid $A D / H D$, anxious, and control children: a meta analysis of studies with the stop task. Journal of Child Psychology and Psychiatry, 39, 41 1-425.

Pennington, B. F., \& Ozonoff, S. (1996). Executive functions and developmental psychopathology. Journal of Child Psychology and Psychiatry, 37, 51-87.

Pierce, C. A., Block, R. A., \& Aguinis, H. (2004). Cautionary note on reporting eta-squared values from multifactor ANOVA designs. Educational and Psychological Measurment, 64(6), 916-924.

Pope, H. G., Gruber, A.J., Hudson, J.I., Huestis, M.A., \& Yurgelun-Todd, D. (2001). Neuropsychological performance in long-term cannabis users. Archives of General Psychiatry, $58,909-915$. 
Rapport, L. J., Van Voorhis, A., Tzelepis, A., \& Friedman, S. R. (2001). Executive functioning in adult attention-deficit hyperactivity disorder. The Clinical Neuropsychologist, 15, 479491.

Reitan, R. M. (1958). Validity of the Trail Making Test as an indicator of organic brain damage. Perceptual and Motor Skills, 8, 271-276.

Rubia, K., Overmeyer, S., Taylor, E., Brammer, M., Williams, S. C., Simmons, A., et al. (1999). Hypofrontality in attention deficit hyperactivity disorder during higher-order motor control: A study with functional MRI. American Journal of Psychiatry, 156, 891-896.

Rucklidge, J. J., \& Tannock, R. (2002). Neuropsychological profiles of adolescents with ADHD: effects of reading difficulties and gender. Journal of Child Psychology and Psychiatry, 43, 988-1003.

Salthouse, T. A. (1996). The processing-speed theory of adult age differences in cognition. Psychological Review, 103(3), 403-428.

Schmidt, M. (1996). Rey Auditory Verbal Learning Test: A handbook. Los Angeles, California: Western Psychological Services.

Seidman, L. J., Biederman, J., Faraone, S. V., Weber, W., \& Ovellette, C. (1997). Toward defining a neuropsychology of attention deficit-hyperactivity disorder: performance of children and adolescents from a large clinically referred sample. Journal of Consulting and Clinical Psychology, 65(1), 150-160.

Seidman, L. J., Biederman, J., Weber, W., Hatch, M., \& Faraone, S. V. (1998). Neuropsychological functioning in adults with attention-deficit hyperactivity disorder. Biological Psychiatry, 44, 260-268.

Seidman, L. J., Doyle, A., Fried, R., Valera, E., Crum, K., \& Mattews, L. (2004). Neuropsychological function in adults with attention-deficit/hyperactivity disorder. Psychiatric Clinics of North America, 27, 261-282.

Seidman, L. J., Valera, E. M., \& Makris, N. (2005). Structural brain imaging of attention deficit/hyperactivity disorder. Behavioral Psychiatry, 57, 1263-1272.

Sergeant, J. A., Geurts, H., \& Oosterlaan, J. (2002). How specific is a deficit of executive functioning for Attention-Deficit/Hyperactivity Disorder? Behavioral Brain Research, $130,3-28$.

Spreen, O., \& Strauss, E. (1998). A compendium of neuropsychological tests: Administration, norms, and commentary. (2 nd ed.). New York: Oxford University Press.

Stone, M., Gabrieli, J. D. E., Stebbins, G. T., \& Sullivan, E. V. (1998). Working and strategic memory deficits in Schizophrenia. Neuropsychology, 12 (2), 278-288.

Stroop, J. R. (1935). Studies of interference in serial verbal reactions. Journal of Experimental Psychology, 18, 643-662.

Stuss, D. T., Floden, D., Alexander, M. P., Levine, B., \& Katz, D. (2001). Stroop performance in focal lesion patients: dissociation of processes and frontal lobe lesion location. Neuropsychologia, 39, 771-786.

Vaidya, C. J., Austin, G., Kirkorian, G., Ridlehuber, H. W., Desmond, J. E., Glover, G. H., et al. (1998). Selective effects of methylphenidate in attention deficit hyperactivity disorder: A functional magnetic resonance study. Proceedings of the National Academy of Sciences of the United States of America, 95, 14994-14999.

Valentijn, S. A., Van Boxtel, M. P., Van Hooren, S. A., Bosma, H., Beckers, H. J., Ponds, R. W., et al. (2005). Change in sensory functioning predicts change in cognitive func- 
tioning: Results from a 6-year follow-up in the Maastricht aging study. Journal of the American Geriatrics Society, 53(3), 374-380.

Van Boxtel, M. P. J., Buntinx, F., Houx, P. J., Metsemakers, J. F. M., Knottnerus, A., \& Jolles, J. (1998). The relation between morbidity and cognitive performance in a normal aging population. Journal of Gerontology, 53A(2), M147-M154.

Van der Elst, W., Van Boxtel, M. P. J., Van Breukelen, G. J. P., \& Jolles, J. (2005). Rey's verbal learning test: Normative data for 1855 healthy participants aged 24-81 years and the influence of age, sex, education, and mode of presentation. Jounal of the International Neuropsychological Society, 11, 290-302.

Van der Elst, W., Van Boxtel, M. P., Van Breukelen, G. J., \& Jolles, J. (2006a). The Stroop Color-Word Test: Influence of age, sex, and education; and normative data for a large sample across the adult age range. Assessment, 13(1), 62-79.

Van der Elst, W., Van Boxtel, M. P. J., Van Breukelen, G. J. P., \& Jolles, J. (2006b). Normative data for the Animal, Profession and Letter M Naming verbal fluency tests for Dutch speaking participants and the effects of age, education, and sex. Jounal of the International Neuropsychological Society, 12, 80-89.

Van der Elst, W., Van Boxtel, M. P. J., Van Breukelen, G., \& Jolles, J. (in press). The Concept Shifting Test: Adult normative data. Psychological Assessment.

van Hooren, S. (2004). Succesful cognitive aging. Executive functioning, determinants, and interventions. Unpublished Dissertation, Maastricht University, Maastricht, the Netherlands.

Vink, M., \& Jolles, J. (1985). A new version of the Trial Making Test as an information processing task. Journal of Clinical Psychology, 7, 162.

Walker, A. J., Shores, E. A., Trollor, J. N., Lee, T., \& Sachdev, P. S. (2000). Neuropsychological functioning of adults with attention deficit hyperactivity disorder. Journal of Clinical and Experimental Neuropsychology, 22, 115-124.

Wechsler, D. (1981). Wechsler Adult Intelligence Scale-Revised. New York: Psychological Corp., Harcourt Brace Jovanovich.

Weiss, G., \& Trokenberg-Hechtman, L. (1993). Hyperactive children grown up. New York: The Guilford Press.

Willcutt, E. G., Doyle, A. E., Nigg, J. T., Faraone, S. V., \& Pennington, B. F. (2005). Validity of the executive function theory of Attention-Deficit/Hyperactivity Disorder: A metaanalytic review. Biological Psychiatry, 57, 1336-1346.

Willcutt, E. G., Pennington, B. F., Olson, R. K., Chhabildas, N., \& Hulslander, J. (2005). Neuropsychological analyses of comorbidity between reading disability and attention deficit hyperactivity disorder: In search of the common deficit. Developmental Neuropsychology, 27(1), 35-78.

Wilens, T. E., Biederman, J., Wozniak, J., Gunawardene, S., Wong, J., \& Monutaux, M. (2003). Can adults with Attention-Deficit/Hyperactivity Disorder be distinguished from those with comorbid Bipolar Disorder? Findings from a sample of clinically referred adults. Society of Biological Psychiatry, 54, 1-8.

Woods, S. P., Lovejoy, D. W., \& Ball, J. D. (2002). Neuropsychological characteristics of adults with ADHD: A comprehensive review of initial studies. The Clinical Neuropsychologist, 16(1), 12-34. 



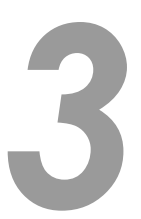

\section{Sustained and focused attention deficits in adult attention deficit hyperactivity disorder (ADHD)}

Natalie D. J. Marchetta, Petra P. M. Hurks, Leo M. J. De Sonneville, Lydia Krabbendam, and Jelle Jolles. Journal of Attention Disorders, in press. 


\section{Abstract}

Objective: To examine the specificity of deficits in focused attention and sustained attention in adults with Attention Deficit Hyperactivity Disorder (ADHD) and to evaluate the impact of comorbidity. Method: Twenty-eight adults with ADHD without comorbidity were compared with 28 ADHD out-patients with comorbidity. Two control groups were used: 68 adults referred for ADHD but with another psychopathology than ADHD (non-ADHD) and 28 healthy controls. All participants completed attention tests of the Amsterdam Neuropsychological Tasks program. Results: Both ADHD groups demonstrated a sustained attention deficit relative to the control groups, as indicated by a disproportionate deterioration of speed fluctuation with time-on-task reflecting temporal lapses in attention. Only the $\mathrm{ADHD}^{+}$group showed focused attention deficits in that they were less able to ignore irrelevant information. Conclusion: These findings show that adults with ADHD have specific deficits in sustained attention. Additional deficits in focused attention are confined to outpatients with ADHD and comorbidity. 


\section{Introduction}

Longitudinal and follow up studies of children with Attention Deficit Hyperactivity (ADHD) suggest that its core features persist into adulthood in at least $30-60 \%$ of cases (Biederman et al., 1998; Manuzza \& Klein, 2000; Weiss \& TrokenbergHechtman, 1993). While some evidence suggests that ADHD involves deficits in attention processes (Tannock, 2003; Van der Meere, Wekking, \& Sergeant, 1991), much of the research on adult ADHD has focused on other domains of cognitive functioning. There has been emphasis on the study of executive function, notably inhibitory processes (see review Hervey, Epstein, \& Curry, 2004). Thus far less is known about the deficits in attention processes associated with adult ADHD. As inattention is a core feature of the disorder (American Psychiatric Association, 1994), especially in adults (Biederman, Mick, \& Faraone, 2000), it is imperative to establish the nature of attention deficits in adults with ADHD. This is the major aim of the present paper.

Over the years attention has been regarded as a multi-factorial construct that encompasses three major conceptualizations: (a) focusing attention, (b) sustaining attention, and (c) shifting attention (Mirsky, Pascualvaca, Duncan, \& French, 1999; Parasuraman, 1998). Focused attention refers to the ability to attend to relevant information while ignoring irrelevant stimuli (Mirsky et al., 1999; Parasuraman, 1998). The most commonly used measures of focused attention involve traditional neuropsychological tests, such as the Stroop-Color-Word Test. Unfortunately, studies comparing ADHD adults with healthy controls using these traditional measures revealed inconsistent results (Hervey et al., 2004; Woods, Lovejoy, \& Ball, 2002). This can be due to several pitfalls. Firstly, these traditional tests typically do not measure unitary cognitive functions (Sergeant, Geurts, \& Oosterlaan, 2002). Secondly, in order to examine the hypothesis of an inhibitory deficit in ADHD, as suggested by some models, most of these studies focused primarily on inhibitory control rather than attention processes (Barkley, 1998; Nigg, 2001). Furthermore, experimental approaches using methods derived from information processing theories were not conducted in recent studies investigating selective attention in adults with ADHD. Such information processing paradigms include precise measures of response latency, variability, and accuracy and are less subject to administration and scoring errors (Tannock, 2003). Some studies examined focused attention in children with ADHD. Several studies used a focused attention task of the Amsterdam Neuropsychological Tasks program by De Sonneville (1999, 2005) (De Sonneville et al., 1994; Hanisch, Konrad, Günther, \& Herpertz-Dahlmann, 2004; Kalff et al., 2005; Konrad, Günther, Hanisch, \& HerpertzDahlmann, 2004). These studies generally found focused attention deficits in children with ADHD relative to controls. Three of these studies documented positive methylphenidate effects in these children with ADHD (De Sonneville et al., 1994; Hanisch et al., 2004; Konrad et al., 2004). Research on focused attention skills in adults with ADHD therefore seems justified. 
With respect to sustained attention, a similar picture emerges. Sustained attention refers to the ability to maintain a stable performance level over time (Mirsky et al., 1999; Parasuraman, 1998). Performance is usually measured in tasks that measure response speed and is expressed by mean reaction time (RT), RT variability (SD of RT), time-on-task (TOT), and errors (Börger et al., 1999). In attention research, the errors of omission (failure to detect the target stimulus) usually reflect inattention symptoms, whereas the commission errors (false alarms) are assumed to reflect a lack of inhibition or impulsivity (Corkum \& Siegel, 1993). Children with ADHD were reported to be impaired in a continuous performance task compared with healthy controls. They showed increased variability, more time-on-task effects, and decreased accuracy (both on omission and commission errors) in their task performance (Börger et al., 1999; De Sonneville et al., 1994; Kalff et al., 2005; Swaab-Barneveld et al., 2000). However, these deficits in attention processes do not appear to be highly specific to children with ADHD because various other clinical groups, such as children with pervasive developmental disorder, conduct disorder, mood and anxiety disorders show similar deficits (Althaus, De Sonneville, Minderaa, Hensen, \& Til, 1996a, 1996b; Pennington \& Ozonoff, 1996; Swaab-Barneveld et al., 2000). It is therefore of interest to evaluate whether a similar picture emerges in adults with ADHD.

Despite the fact that most previous research on ADHD has focused on children, there is growing interest in investigating attention deficits in ADHD adults. To date, a number of studies as well as meta-analytic reviews examined sustained attention. These are usually measured by continuous performance tasks (CPTs) in controlled designs in which adults with ADHD are compared with healthy controls (Barkley, Murphy, \& Kwasnik, 1996; Epstein, Conners, Sitarenios, \& Erhardt, 1998; Epstein et al., 2001; Murphy, Barkley, \& Bush, 2001; Ossman \& Mulligan, 2003). These studies reported impaired performance in terms of reaction time (RT), variability of RT, and response accuracy as measured by errors of commission and omission. Reaction time variability is considered an important parameter in evaluating sustained attention deficits because it is a measure of performance stability. The evaluation of performance with time-on-task (TOT) effect is also important. The TOT effect is a crucial marker of sustained attention (Van der Meere \& Sergeant, 1988), yet it was usually not measured or neglected in earlier studies. Research on the various aspects of attention in adults with ADHD is therefore warranted. Measures relating to both focused and sustained attention should be taken.

Comorbidity is an additional factor that studies into adult ADHD should encompass in view of its importance in children. There is an extremely high rate of comorbidity in adults with ADHD. For instance, in $50-80 \%$ of cases ADHD occurs simultaneously with other psychiatric problems and/or diagnoses, such as antisocial and other personality disorders, substance abuse, anxiety, and mood disorders (Biederman, Newcorn, \& Sprich, 1991; Marks, Newcorn, \& Halperin, 2001). Some studies on children with ADHD found that the neuropsychological deficits 
of children with concurrent ADHD and a comorbid disorder (i.e., conduct disorder, anxiety disorder, oppositional defiant disorder, reading disorder) are worse than those of children with ADHD only (Swaab-Barneveld et al., 2000; Willcutt, Pennington, Olson, Chhabildas, \& Hulslander, 2005). In adults with both ADHD and a comorbid disorder, it is important to determine whether cognitive deficits, such as attention deficits, are the result of co-occurring symptomatology or ADHD itself.

In summary, the aims of the present study were threefold. First, focused and sustained attention processes were examined by computerized attention tasks in adults with ADHD only. Second, time-on-task performance on a sustained attention task was examined in this study. Third, the specificity of the focused and sustained attention profile and the impact of comorbidity were investigated by including three control groups, namely an ADHD with comorbidity group, a nonADHD group, and a healthy control group. Based on the previous studies, we first hypothesized that the ADHD adults would show impairments on the focused and sustained attention tasks and that the impairments would be more pronounced in the ADHD with comorbidity group. We also hypothesized that if the performance of the ADHD groups with and without comorbidity was significantly different from the performance of the other two groups, the impairments would be attributable to ADHD. If the performance of the ADHD with comorbidity group and the non-ADHD group were significantly different from the performance of the ADHD without comorbidity group, the impairments would be attributable to the comorbidity.

\section{Method}

\section{Participants}

The patients in this study were referred to a specialized outpatient health-care setting in a general psychiatric hospital (Psychomedical Center Vijverdal Maastricht, in the southern Netherlands). At the time of referral these individuals had complaints and or a medical history suggestive of adult ADHD. Referral was always by general practitioners or medical specialists. There were three outpatient groups, namely individuals with ADHD without comorbidity (ADHD; $n=28$ ), ADHD individuals with comorbidity (ADHD+; $n=28$ ), and outpatients with complaints in the domain of ADHD but other psychiatric diagnosis (non-ADHD; $n=68$ ) and can be regarded as consecutive referrals assessed from 1998 to 2003.

A multidisciplinary workup was used, as detailed in other studies (e.g., Barkley, Murphy, \& Bush, 2001). Briefly, the clinical assessment consisted of a standard diagnostic and research protocol, including a semi-structured diagnostic interview and a clinical interview with both the patient and a cross-informant (usually a parent or spouse). In these interviews the following topics were collected: (1) demographic, academic and work history, (2) the developmental cour- 
se, (3) the ADHD symptoms and criteria according to DSM-IV, but also other cognitive complaints, and (4) the comorbidity psychopathology. The interviewer rated then if a symptom was present or not. In addition, self-report rating scales, including a Dutch version of the Wender Utah Rating Scale (WURS; Ward, Wender, \& Reimherr, 1993) and a Dutch version of the ADHD Rating Scale based on the DSM-IV criteria, including recall of current symptoms and retrospective report during childhood (APA, 1994) were moreover completed and used as additive experimental diagnostic tools. A neuropsychological examination (e.g., intelligence, attention) and a review of school reports, if available, were also part of the assessment. Diagnosis and classification of ADHD was made by a multidisciplinary health care team, including psychiatrists and licensed health care psychologists, and was based on a clinical consensus in which all the data mentioned above were judged. It is worth noting that the clinical neuropsychological evaluation was always part of the assessment, but the information it contained can be regarded as a supplementary tool in the diagnostic process. All individuals in the ADHD- group met the diagnostic criteria for ADHD according to the Diagnostic and Statistical Manual of Mental Disorders (APA, 1994), including current symptoms and a retrospective diagnosis of childhood ADHD. All individuals in the $\mathrm{ADHD}^{+}$group met criteria for ADHD and also DSM-IV criteria for mood disorders $(n=9)$, anxiety disorders $(n=1)$, mood and anxiety disorder $(n=1)$, substance-related disorders $(n=6)$, personality disorders $(n=10)$, and a combination of mental disorders (mood disorders, PTSS, psychosis NOS) with a borderline personality disorder $(n=1)$. The individuals in the non-ADHD group presented with complaints possibly reminiscent of manifestations of ADHD but did not meet the criteria for ADHD. Individuals in this group were diagnosed with personality disorders $(n=20)$, a combination of DSM-IV mental disorders (mood, anxiety disorder or substance-related) with personality disorders $(n=11)$, mood disorders $(n=5)$, anxiety disorders $(n=4)$, mood and anxiety disorders $(n=1)$, oppositional disorder $(n=1)$, schizophrenia $(n=1)$, borderline ADHD (some symptoms of ADHD but less than six) with a personality disorder or a substance-related disorder $(n=6)$, mental retardation (Axis-2) $(n=1)$, burn-out $(n=1)$, and no DSM-IV diagnosis $(n=$ 17). Participants using short-acting stimulant medication (e.g., methylphenidate) for ADHD were not excluded from the present study. They were asked to cease in general their medication 24 hours before the testing.

The healthy control group consisted of 28 individuals who responded to advertisements in a local newspaper and notices in Maastricht University Hospital and Maastricht University. Control participants took part in a telephone screening and completed a self-report rating on ADHD. Exclusion criteria for this sample were: (1) current regular use of medication and/or drugs, (2) IQ below 85; (3) selfreported history of a psychiatric disorder (including ADHD), (4) active medical or neurological diseases, such as a serious head trauma, seizures, and diabetes mellitus, and (5) learning disabilities. 
Participant characteristics of the ADHD and comparison groups are represented in Table 1.

Table 1. Participant characteristics of the four groups

\begin{tabular}{lcccccc}
\hline Variable & $\begin{array}{c}\text { ADHD } \\
(n=28)\end{array}$ & $\begin{array}{c}\text { ADHD+ } \\
(n=28)\end{array}$ & $\begin{array}{c}\text { Non- } \\
\text { ADHD } \\
(n=68)\end{array}$ & $\begin{array}{c}\text { Controls } \\
(n=28)\end{array}$ & $F$ & $X^{2}$ \\
\hline Gender (\% women) & 39.3 & 25 & 33.8 & 50 & & 4.11 \\
Medication (\% yes) & 25 & 32.1 & 52.9 & 0 & & $26.26^{* *}$ \\
Age (M, SD) a & 33.80 & 32.36 & 32.22 & 28.87 & 1.72 & \\
& $(9.64)$ & $(8.23)$ & $(10.49)$ & $(6.51)$ & & \\
IQ (M, SD) & 108.43 & 103.46 & 101.93 & 109.93 & $2.94^{*}$ & \\
& $(10.81)$ & $(13.95)$ & $(15.17)$ & $(13.43)$ & & \\
Education $(M, S D)^{b}$ & 4.00 & 4.11 & 3.38 & 5.11 & $8.30^{* *}$ & \\
\hline
\end{tabular}

Note. aThe age range was 19 to 62 . ${ }^{b}$ Measured on an 8-point scale ranging from primary school (1) to university degree (8) (De Bie, 1987). ${ }^{*} p<.05 .{ }^{* *} p<.001$.

\section{Measures}

To measure attention, all participants performed a baseline speed, a focused and a sustained attention task of the well-validated Amsterdam Neuropsychological Tasks (ANT) program (De Sonneville, 1999, 2005). The ANT is a computeraided assessment battery of reaction time tasks that allows for the systematic evaluation of information processing capacities. Ample studies have proven the ANT to be a sensitive and valid tool in non-referred samples (e.g., Brunnekreef, 2007), as well as in referred samples, such as attention deficit disorders (e.g., Konrad et al., 2004; Slaats-Willemse, Swaab-Barneveld, De Sonneville, \& Buitelaar, 2005). Test-retest reliabilities for the ANT have also been found to be satisfactory (De Sonneville, 2005; Günther, Herpertz-Dahlmann, \& Konrad, 2005). All participants provided written informed consent. The three attention tasks used for the present paper were selected to directly address the study topics and were administered in the order listed.

Task one: Baseline Speed task. A cross is displayed in the centre of the computer screen as a fixation point, which may change into a white square (Figure la, top left). When this change occurs, participants should press the mouse key as fast as possible, after which the cross returns and this sequence repeats itself. The post-response interval (PRI: period between response and next stimulus on-set) varied randomly between $500-2500 \mathrm{~ms}$ to prevent anticipation strategies. This task consists of 10 practice trials and 32 real trials for each hand, starting with the non-dominant hand followed by the dominant hand. This task is known to measure simple speed, which requires minimal cognitive demands and can therefore be used as a measure for simple automated motor reaction. The following two main outcome measures were chosen: 
1. Speed. Median reaction times for baseline speed (in ms; averaged over both hands) were calculated as an index of speed of information processing.

2. Speed variability. Within-subject standard deviations of reaction times of baseline speed (averaged over both hands) were calculated as a measure of speed variability.

Task two: Focused Attention task (Figure 1c, bottom). During this task two diagonally placed letters are presented on the screen. Although the two letters can appear on either one of the two diagonals, the participant is asked to press the 'yes' key only in response to the letter I (target letter) on the relevant axis upper-left or lower-right positions), and to press the 'no' key in all other cases. The 'yes' key and 'no' key correspond to the right and left mouse button respectively for right-handers, and vice versa for left-handers. The fixed PRI was 1200 ms and the stimulus duration was $300 \mathrm{~ms}$. In this task 60 relevant targets (letter I on relevant axis), 20 relevant non-targets (non-target letters on relevant axis), 20 irrelevant targets (letter I presented on irrelevant axis), and 20 irrelevant non-tar-gets (non-target letters on irrelevant axis) are presented in a random lorder. This task was chosen in order to evaluate the ability to discriminate relevant from irreleivant information, i.e., the ability to focus attention. The following three main outcome parameters were included:

1. Speed. Median reaction times determined for relevant targets, relevant non-targets, irrelevant targets and irrelevant non-targets (all in ms) were used as measures of speed of information processing.

2. Speed variability. The average of the within-subjects standard deviations of reaction times for all four possible correct responses was calculated as a measure of speed variability.

3. Accuracy. The percentages of false alarms (to irrelevant targets, relevant non-targets, irrelevant non-targets) and misses (of relevant targets) were calculated separately to obtain the error percentages as an index of accuracy.

Task three: Sustained Attention task. This task is a variant of a widely used continuous performance task. During this task a square with three, four or five dots is continuously depicted on the screen. The participant is required to press the 'yes' button if four dots (target) are presented and to press the 'no' button whenever three or five dots (non-targets) are presented (Figure 1b, top right). In this task 50 series of 12 items, in total 600 items, are presented according to a balanced presentation of four signals of each type $(3,4$, and 5 dot patterns) in random order in each series. It was a self-paced task, with a PRI of $250 \mathrm{~ms}$ and it takes about 15 minutes to complete this task. The main parameters were:

1. Speed (tempo). Mean completion time per series (MST) was calculated as an index for tempo of sustained attention performance. Mean tempo per block of 10 series (five periods) was computed. 
a.Baseline speed

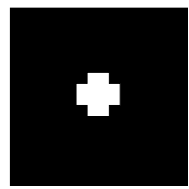

fixation

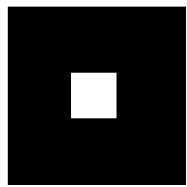

signal b. Sustained attention

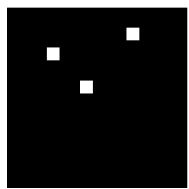

3 dots non-target

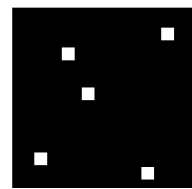

5 dots non-target

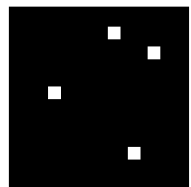

4 dots target

c. Focused attention

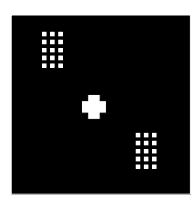

relevant axis

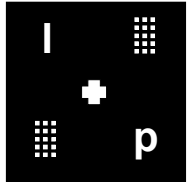

relevant target

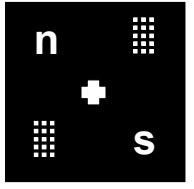

relevant non-target

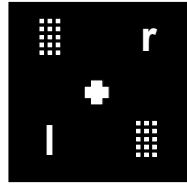

irrelevant target

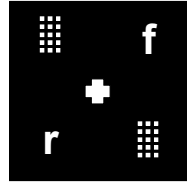

irrelevant non-target

Figure 1. Example of all possible signal types in the Baseline Speed, the Sustained Attention, and the Focused Attention tasks

2. Speed variability. Within-subject standard deviations of mean series time (SMST) (across 50 series) were calculated as a measure of fluctuation in tempo.

3. Accuracy. The percentage of misses (pressing the 'no' button for 4 dots) and the percentage of false alarms (pressing the 'yes' button for 3 or 5 dots) were calculated as an index of accuracy of task performance. The balanced presentation (equal number of 3, 4, and 5 dots) paradigm will invoke a response bias in the participants as they should press the 'no' button twice as often as the 'yes' button. That is, the less participants are capable of inhibiting biased response tendencies, the more misses they will produce relative to false alarms with time-on-task (De Sonneville et al., 1994). Thus, in this task the number of misses constitutes an index of response disinhibition; and false alarms reflect inadequate stimulus evaluation.

4. Time-on-Task. Changes in performance with time-on-task (TOT) were computed by dividing the 600 responses in blocks of 10 series (5 periods). Speed (RT) and fluctuation in speed (SD of RT) were calculated for the mean of the first two periods (block 1) and the last two periods (block 2). Finally, to study accuracy over time, the percentages of misses and false alarms for the first $40 \%$ of responses (mean of first two periods) and last $40 \%$ of the responses (mean of last 2 periods) were calculated. A disproportionate number of misses with time-on-task was taken as an indication of a failure to sustain inhibition of prepotent responses. 
Estimated intellectual functioning. Estimated IQ was assessed for all participants with the age-related shortened form of a widely used Dutch intelligence test, the Groningen Intelligence Test (GIT) (Luteijn \& van der Ploeg, 1983). There is general agreement that the use of the three subtests (Arithmetic, Mental Rotation, Analogies) yields a good approximation of a full-scale IQ $(M=100 ; S D=15)$ (Luteijn \& van der Ploeg, 1983).

\section{Data analysis}

Prior to all analyses the three tasks were examined separately for extreme values in median reaction times and within-subject standard deviations (> 3 SD from within-group median value) and for error percentages $\geq 50 \%$. Several participants had to be excluded because of this. In addition, some participants had to be excluded for particular tasks because they did not complete the task. The number of participants excluded per variable ranged from 2 (for the four groups together, $N=148$ ) to 17 . The number of degrees of freedom in the statistical tests consequently varied somewhat for the various groups. All participants with missing data or extreme values were pairwise excluded from the ANOVA analyses for the outcome measures and they were listwise excluded for the repeated measures analyses.

Overall task performance of the four groups in terms of the main outcome measures of speed and speed variability were analyzed with General Linear Model (GLM) univariate ANOVA analyses per task. Accuracy on the Focused and Sustained Attention tasks and TOT effects were analyzed by GLM repeated measures analyses. Greenhouse-Geisser correction probabilities were used to deal with unequal sample sizes and for violation of Mauchly's Test of Sphericity. Additionally, bivariate correlation analyses between the outcome measures and demographic variables (see Table 1) were made in order to investigate the possible effect of these confounding variables. In case of a significant correlation, these confounding demographic variables were included in a second step as covariates in the analyses.

Statistical significance was set at $p \leq .05$, two-tailed, for all comparisons. Significant group differences were analyzed post hoc using Fischer's Least Significant difference with $a=.05$. Simple contrasts were used in order to test our planned comparisons (ADHD- vs. NC, ADHD- vs. non-ADHD, ADHD- vs. ADHD+, $\mathrm{ADHD}^{+}$vs. NC, $\mathrm{ADHD}^{+}$vs. non-ADHD). In an effort to better identify the strength of the associations, we used partial eta squared $\left(n p^{2}\right)$ values as an estimate of effect size as reported by the SPSS output files (Pierce, Block, \& Aguinis, 2004). Values for $\eta_{p}{ }^{2}$ approximately correspond to the following effect size conventions: small (0.01), medium (0.06), and large (0.14) (Cohen, 1988). All statistical analyses were performed using SPSS for Windows 11.0. 


\section{Results}

\section{Demographic characteristics}

As can be seen in Table 1, significant group differences were found with respect to medication, $I Q$ and education. A significantly higher percentage of nonADHD adults were (recently) taking medication, including antidepressants and anxiolytics. Also, the non-ADHD group had a lower IQ than the healthy controls and ADHD adults ( $p<.05$ ), and educational level was significantly lower in the non-ADHD adults than in the healthy controls and the ADHD+ adults $(p<.05)$. The groups were equivalent in gender and age.

\section{Baseline Speed task}

GLM univariate analysis of the median reaction times revealed a significant main effect of group, $F(3,139)=5.34, p=.002, n p^{2}=.103$ (see Figure 2). Simple contrasts indicated that the $A D H D$ - adults reacted faster than the healthy controls ( $P$ $=.001$ ) and $\mathrm{ADHD}^{+}$adults ( $p=.007$ ), but no differences were found between the ADHD- adults and non-ADHD adults. The ADHD+ adults were also slower than the non-ADHD adults ( $p=.027$ ), but not in comparison with healthy controls. There was a significant negative correlation between medication and Speed $(r=-.178$, $p=.024)$. Univariate analyses with medication as a covariate yielded comparable results.

Univariate GLM analyses with the standard deviations of median reaction times of Baseline Speed resulted in no significant effect of group, $F(3,138)=1.89$, $p=.134, \eta p^{2}=.04$. As simple speed variability correlated significantly with education ( $r=-.25, p=.001$ ), univariate GLM analysis with education as a covariate was performed. The results were comparable after correcting for education and thus indicating no group differences in simple speed variability.

\section{Focused Attention task}

A $2 \times 2 \times 4$ (group) GLM repeated measures analysis with location (relevant vs. irrelevant axis) and type of stimulus (target letter vs. non-target letter) as withinsubject factors and group as between-subject factor revealed significant withinsubject main effects of location, $F(1,126)=11.07, p=.001, \eta_{p}^{2}=.081$, type of stimulus, $F(1,126)=29.44, p<.001, \eta_{p}^{2}=.189$, and a location by type of stimulus interaction, $F(1,126)=218.08, p<.001, \eta_{p}^{2}=.634$. This indicated that the four groups identified target letters faster than non-target letters when these were presented on the relevant axis, whereas the reverse was found when target letters were presented on the irrelevant axis. This result confirms the focused attention model paradigm with appropriate exhaustive search on the relevant axis and time-consuming distraction by presentation of irrelevant target letters. There were no significant interactions between the four groups and the withinsubject factors. Also, there was no main effect of group on speed, $F(3,126)=$ $0.36, p=.779, \eta p^{2}=.009$. 


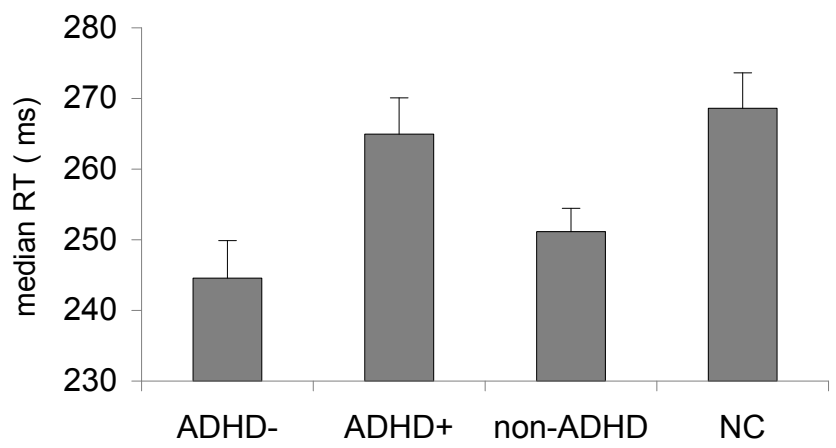

Figure 2. Speed of processing for the Baseline Speed task Note. $\mathrm{NC}=$ healthy controls

A significant main effect of group, $F(3,134)=4.06, p=.009, \eta_{p}^{2}=.086$, resulted for variability in reaction time. The $\mathrm{ADHD}^{+}$adults and non-ADHD adults had a significantly higher variability in speed than ADHD- adults and healthy controls (see Figure 3). Because speed variability correlated significantly with IQ $(r=-.396$, $p<.001$ ), a GLM univariate analysis with IQ as a covariate was performed. Results revealed a main effect of $I Q(p<.001)$ in combination with an attenuation of the group effect $\left(p=.112, \eta_{p}^{2}=.045\right)$.

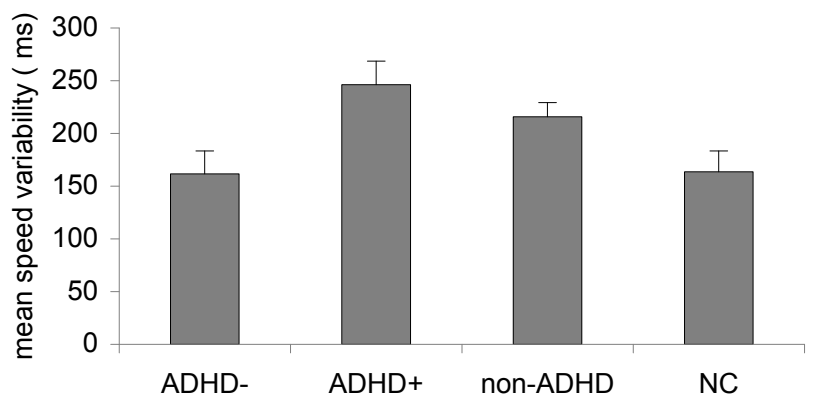

Figure 3. Mean speed variability on the Focused Attention task Note. $\mathrm{NC}=$ healthy controls

The $2 \times 2 \times 4$ (group) GLM repeated measures with location and stimulus type as within-subject factors and group as a between-subject factor for accuracy revealed a significant effect of location, $F(1,129)=29.21, p<.001, \eta_{p}^{2}=.185$, and a location by stimulus type interaction, $F(1,129)=54.32, p<.001, \eta p^{2}=.296$. The presence of target letters on the irrelevant axis resulted in more errors than the presence of non-target letters, while the reverse was true for the relevant axis. In addition, a significant main effect of group was found, $F(3,129)=5.02, p=$ $.003, \eta_{p}^{2}=.105$ (see Figure 4). The ADHD+ adults were less accurate than the heal- 
thy controls, non-ADHD adults, and ADHD- adults (all $p s<.01$ ). After adjusting for $I Q$ ( $r$ range with outcome measures $=-.195-.419, p<.05)$, comparable results were found.

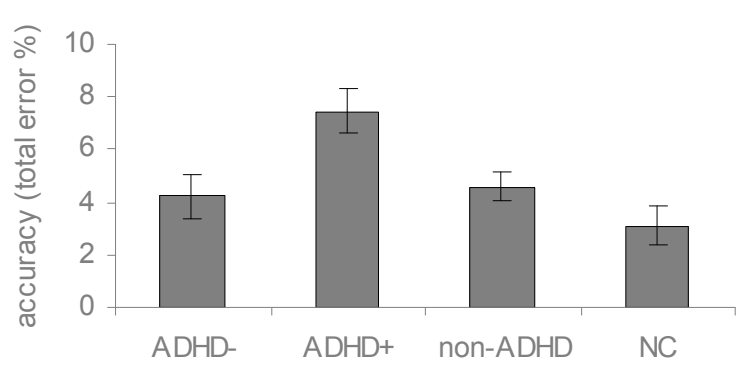

Figure 4. Percentage of errors (\%) for accuracy on the Focused Attention task Note. $\mathrm{NC}=$ healthy controls

Sustained Attention task

A trend of group effect was found for mean tempo (MST), $F(3,143)=2.58, p=$ $.056, \eta_{p}{ }^{2}=.051$. The $\mathrm{ADHD}^{+}$adults were in general slower than the non-ADHD adults $(p=.013)$ and healthy controls $(p=.031)$. As IQ correlated significantly with MST $(r=-.326, p<.001)$, an ANCOVA with IQ as a covariate was done. The results showed a main effect of group $(p=.027)$, indicating that the ADHD+ adults $(p=$ .006 ) and ADHD- adults ( $p=.047$ ) were slower than the non-ADHD adults. GLM repeated measures analysis of mean completion time per series with TOT (block 1 vs. block 2) as within-subject factor and group as between-subject factor revealed no significant TOT effect and no group by TOT interaction. This indicated that the $\mathrm{ADHD}^{-}$and $\mathrm{ADHD}^{+}$adults did not differ from healthy controls and non-ADHD adults regarding changes in tempo with TOT. A close inspection of the data revealed that the $\mathrm{ADHD}^{+}$, non-ADHD adults, and healthy controls became faster in the second block of the task, but this did not reach significance (see Figure 5).

Univariate GLM analysis of mean fluctuation in tempo (SMST) revealed significant group differences, $F(3,142)=4.019, p=.009, \eta_{p}^{2}=.078$. The ADHDadults demonstrated a larger fluctuation than the healthy controls $(p=.026)$, and the $\mathrm{ADHD}^{+}$adults had a larger fluctuation in tempo than the healthy controls $(p=$ .003 ) and non-ADHD adults ( $p=.011)$. GLM repeated measures analysis of fluctuation in tempo with TOT (block 1 vs. block 2) as within-subject factor and group as between-subject factor revealed a significant TOT effect, $F(1,142)=21.076, p$ $<.001, \eta_{p}^{2}=.129$, and a significant TOT by group interaction, $F(3,142)=2.73, p=$ $.046, \eta_{p}^{2}=.055$ (see Figure 5). Inspection of Figure $5 \mathrm{~b}$ reveals that, on average, fluctuation in tempo increases with time-on-task, but this applies in particular to both ADHD groups. As IQ correlated significantly with SMST $(r=-.312, p<$ 
.001 ), an ANCOVA with IQ as a covariate was performed. The same results were found for the TOT by group interaction and main effect of group, but the TOT effect $(p=.397)$ was attenuated.
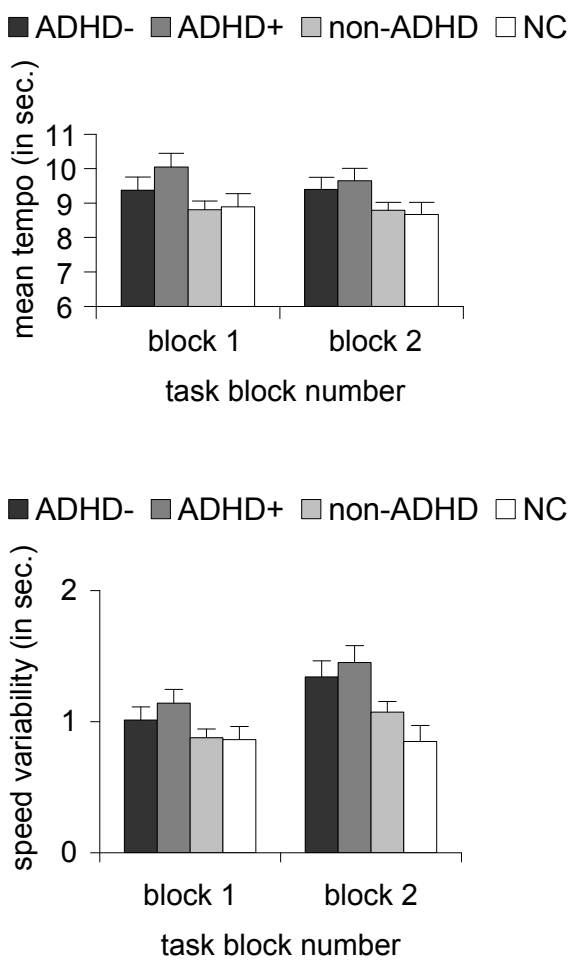

Figure 5. Mean tempo (and SE) (Figure 5) and speed variability (Figure 5) for the first two periods (block 1) and last two periods (block 2) on the Sustained Attention task Note. $\mathrm{NC}=$ healthy controls

A $2 \times 4$ (group) GLM repeated measures analysis of accuracy with error type (misses vs. false alarms) as within-subject factor and group as betweensubject factor revealed a significant effect of error type, $F(1,146)=106.11, p<$ $.001, \eta_{p}^{2}=.423$, but no error type by group interaction, $F(3,146)=1.24, p=.297$, $\eta_{p}{ }^{2}=.025$. This indicated that participants made more misses $(7.5 \%)$ than false alarms (3\%) and confirms the development of a response bias within the four groups. No significant effect of group was found, $F(3,146)=2.18, p=.093, \eta_{p}^{2}=$ .043. A $2 \times 2 \times 4$ GLM repeated measures analysis with error type and TOT (block 1 vs. block 2) as within-subject factor, and group as between-subject factor revealed a significant TOT effect, $F(1,146)=40.90, p<.001, \eta_{p}^{2}=.219$, a significant effect of error type, $F(1,146)=93.63, p<.001, \eta p^{2}=.391$, and a TOT $\times$ error type interaction, $F(1,146)=23, p<.001, \eta p^{2}=.136$. This indicated that the percentage of errors increased with TOT within the four groups and this was mainly true 
for the percentage of misses (see Figure 6). Simple contrasts revealed that the ADHD ${ }^{+}$adults $(p=.022)$ and ADHD- adults $(p=.056)$ were less accurate than the healthy controls, but this has to be interpreted with caution as no interaction of error type or TOT with group was found.

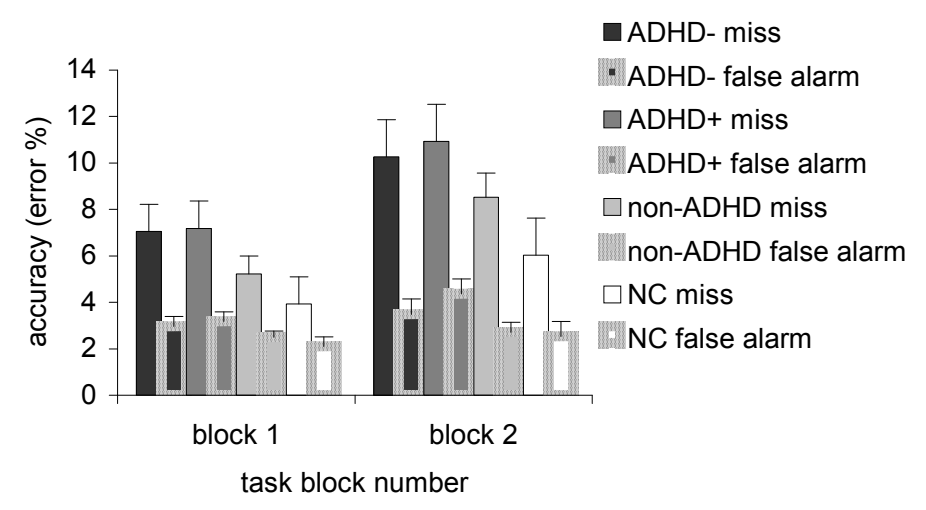

Figure 6. Percentage of misses and false alarms (\%) (and SE) of the first two periods (block 1) and last two periods (block 2) on the Sustained Attention task Note. $\mathrm{NC}=$ healthy controls

\section{Discussion}

In general, with respect to our hypotheses, we found that the ADHD outpatients with and without comorbidity were slower and more variable in their tempo over time on a sustained attention task. This indicates that sustained attention deficits were specific to ADHD regardless of comorbidity. In addition, the ADHD outpatients with comorbidity were only impaired with respect to focusing attention. More specifically, they were less accurate than the other three groups on a focused attention task, indicating that the combination of ADHD with comorbidity is more confined to problems with focusing attention. These findings also indicate that adults with ADHD and comorbidity are characterized by different attention problems. When adjusted for $I Q$ and medication usage, the findings remained essentially the same. These results warrant a more detailed discussion per task.

\section{Baseline Speed}

The hypothesis that no group differences would be found on this task was supported with respect to simple speed variability, but not with respect to speed. The ADHD- adults were significantly faster than the $\mathrm{ADHD}^{+}$adults and healthy 
controls, also when medication usage was taken into account. This suggests that there is a small but distinct difference in simple information processing between adults with ADHD with and without comorbid disorders. Because ADHD+ adults could not be differentiated from healthy controls, this finding cannot be seen as clinically significant. This finding is consistent with earlier research on adult ADHD as reviewed by Hervey et al. (2004), who reported small effect sizes and no performance differences between ADHD adults and healthy controls in terms of processing and speed outcome measures that require minimal processing demands.

\section{Focused Attention}

The hypothesis regarding focused attention deficits in adults with ADHD was partially confirmed. The ADHD- adults did not differ from the healthy controls in speed (processing time), speed variability, and accuracy, indicating that there is no deficit in focused attention in these ADHD-adults. Interestingly, this result shows that attention processes and performance is different in adults as against children with ADHD. Previous studies with children with ADHD revealed other findings although the same focused attention paradigm was used (De Sonneville et al., 1994; Hanisch et al., 2004; Kalff et al., 2005). Other studies on adult ADHD reported inconsistent findings regarding focused attention (Hervey et al., 2004; Woods et al., 2002). This is likely due to the fact that these studies generally used traditional tests, such as the Stroop-Color-Word Test. These tests are known to measure not only focused attention but also other processes, such as inhibition and interference control. Therefore, results generated by these tests must be interpreted with caution (Sergeant et al., 2002).

When examining the specificity of the focused attention deficits, a pattern of clear group differences emerged. The ADHD+ adults were less accurate than the healthy controls, but also less accurate than the ADHD- adults and non-ADHD adults. Post hoc comparisons of the three false alarms types and misses separately revealed a significant main effect of group on false alarms for irrelevant targets $(p<.01)$. The $\mathrm{ADHD}^{+}$adults made significantly more false alarms for irrelevant targets than the three other control groups. This finding indicates that ADHD adults with comorbidity have specific problems in inhibiting a response to irrelevant information on a focused attention task that involves high controlled processing demands. This is a relatively new finding as the presentation of such a focused attention task to adults with ADHD has no precedent in the literature. In addition, we also found that the comorbid ADHD outpatients had a higher variability in speed than healthy controls and ADHD outpatients without, although checking for $I Q$ attenuated this effect. This underscores the importance of the intermediating IQ factor in ADHD symptomatology. These findings suggest that the ADHD adults with comorbidity show a different focused attention pattern than ADHD- adults and adults referred for ADHD but with another psychopathology than ADHD (non-ADHD). It can be concluded that specific deficits in fo- 
cused attention in the ADHD comorbid group are due to comorbidity more than to the ADHD symptomatology. The $\mathrm{ADHD}^{+}$adults had a more complex psychopathology and were characterized by more severe psychiatric symptoms. This finding suggests that comorbid psychopathology may be more important for the expression of ADHD symptomatology and mental health problems than was previously thought. More research could contribute to a better understanding and differentiation of behavioral patterns, especially in the domain where patients present with neurocognitive complaints and related problems yet may have underlying pathology relating to affective aspects and other forms of psychopathology.

\section{Sustained Attention}

On the whole, compared with healthy controls and non-ADHD adults, the ADHD outpatients with and without comorbidity were somewhat slower and demonstrated larger fluctuations in speed. We found that the ADHD- adults were not different in speed compared with healthy controls. This finding is consistent with previous studies that compared ADHD adults with healthy controls and revealed no group differences on the CPT hit RT measure (Epstein et al., 1998; Epstein et al., 2001; Murphy et al., 2001).

However, with respect to the specificity of speed of performance, our results revealed that the $\mathrm{ADHD}^{+}$adults were generally slower from the very beginning of the task than the healthy controls and non-ADHD controls, but no TOT-related decrease in tempo was found in these $\mathrm{ADHD}^{+}$adults. The $\mathrm{ADHD}^{+}$ adults, non-ADHD and healthy controls even became faster with TOT, but this change was not significant. This finding is consistent with previous studies that compared children with ADHD with other clinical and healthy controls with regard to sustained attention tasks and found no TOT decrease in reaction time (Börger et al., 1999; Kalff et al., 2005; Swaab-Barneveld et al., 2000). This absence of TOT decrease in speed in ADHD adults can partly be explained as an effect of the task paradigm that invokes a response bias which induces faster response at the expense of accuracy. As the bias increases with TOT, speed may not decrease, at the cost of an increase in misses with time-on-task. In contrast to our expectation, the ADHD adults did not show a larger response bias and were not less accurate. This indicates that the ADHD- and $\mathrm{ADHD}^{+}$adults were capable of inhibiting prepotent responses to the same extent as healthy controls and nonADHD controls. This interesting finding is not consistent with results found in children with ADHD. These children were found to be more susceptible to the effect of a response bias (De Sonneville et al., 1994; Swaab-Barneveld et al., 2000) and were also characterized by higher numbers of misses (De Sonneville et al., 1994; Swaab-Barneveld et al., 2000). Likewise, there was some research in adults with ADHD that reported more commission errors (e.g., measure for inhibition) (Epstein et al., 1998; Epstein et al., 2001; Murphy et al., 2001). In general, our finding does not confirm the inhibition hypothesis that Barkley (1998) 
stated for children. According to this notion, response inhibition is the core deficit in the wide pattern of cognitive impairments in children with ADHD. Some studies confirmed a deficit in response inhibition in adults with ADHD (Epstein et al., 2001; Murphy et al., 2001; Rapport et al., 2001), but this does not extend to our study. Therefore, further research on elaboration of these models is necessary.

Furthermore, regarding speed variability, the ADHD- adults showed greater fluctuations in speed. The increase in speed fluctuation with TOT was also larger compared with healthy controls who had a tendency to show a decrease or no change in fluctuation. This parameter has barely been investigated in adults with ADHD. Most other studies used CPTs in studying adult ADHD that reported overall higher speed variability (RT SD) in adults with ADHD relative to healthy controls (Barkley et al., 1996; Epstein et al., 2001; Murphy et al., 2001; Walker et al., 2000). These studies failed to report whether changes in performance with TOT discriminate between these groups. A change (deterioration) in fluctuation with TOT is considered an important marker of sustained attention deficit and reflecting temporal lapses in attention, which is even more appropriate than overall RT variability (Castellanos \& Tannock, 2002; De Sonneville et al., 1994; Van der Meere \& Sergeant, 1988). Support for the present finding is also provided by previous studies that investigated the effects of nicotine treatment in adults with ADHD (Levin, Conners, Silva, Canu, \& March, 2001). Their results indicated that acute and chronic nicotine treatment significantly reduced variability in reaction time over trial blocks on a CPT in adults with ADHD. Our results with respect to speed (RT) variability with TOT are also consistent with similar research conducted in previous pediatric populations (Epstein et al., 2003; Kalff et al., 2005; SwaabBarneveld et al., 2000). The present results therefore extend these findings to adults with ADHD. This increased variability in speed in adults with ADHD suggests that the underlying problem in these adults may be a difficulty in maintaining an optimal level of performance or lack of consistent effort, which has also been reported in children with ADHD (Börger et al., 1999; Kuntsi, Oosterlaan, \& Stevenson, 2001). With respect to the specificity of speed variability in adults with $\mathrm{ADHD}$, it is noted that the $\mathrm{ADHD}^{+}$adults also had an enlarged fluctuation in speed over time compared with healthy controls and non-ADHD adults, suggesting that this TOT-related deficit is specific for adult ADHD. In general, few studies have controlled for the effect of comorbid conditions. Additionally, few studies have used an ADHD group with comorbidity and clinical non-ADHD controls as comparison groups to delineate specific ADHD-related neuropsychological deficits with computerized attention tasks (Hervey et al., 2004). However, Murphy et al. (2001) did address the effects of comorbidity on neuropsychological measures (including the CPT). On the basis of a cohort control approach, they found that the additional presence of comorbid symptomatology did not influence neuropsychological performance. This finding is consistent with our results regarding the sustained attention task. In contrast, Hervey et al. (2004) demonstrated, in their meta-analytic review, that ADHD patients with comorbid 
disorders usually demonstrated greater neuropsychological deficits than those with ADHD only. This finding is partially consistent with our focused attention findings that are specific for adults with comorbid ADHD. Future research in which these groups of ADHD subjects with and without comorbidity are compared is therefore necessary to provide further evidence on the specificity of speed variability in adults with ADHD. In summary, with respect to our second objective, we found that fluctuation in speed over time is an important and specific marker for sustained attention deficits in both ADHD only adults and ADHD adults with comorbidity in regard to which they did not differ. Likewise, future studies assessing differences between ADHD subtypes in relation to attention deficits will shed more light on the potential differential mechanisms involved in adult ADHD that were beyond the scope of this paper.

\section{Limitations of the study}

Several possible limitations of the study merit consideration. Some concerns exist with respect to the approach taken to investigate the impact of comorbidity. The impact of comorbidity was analysed by comparing a pure ADHD group with two heterogeneous clinical control groups. ADHD symptom severity as well as the range and heterogeneity of comorbidity were not controlled in all respects. Accordingly, the results do not enable inferences on the impact of the severity and nature of the various kinds of comorbidity. However, despite the fact that this could yield additional and relevant information, symptom severity and the range of comorbid disorders were not the primary focus of this study. Nonetheless, we recommend that future studies both control for ADHD symptom severity and include various but separate comorbid disorders together with ADHD and clinical controls. By doing this, it will be possible to delineate the extent to which attention test performance varies as a function of specific comorbid patterns.

A second limitation of this study relates to the treatment history of adult ADHD and non-ADHD outpatients. Although we used a 24 hour wash-out period before testing, it is possible that this period may not have been sufficiently adequate to control for the possible effects of stimulant medication. However, previous studies have also used a 24 hour washout procedure (Murphy et al., 2001), and the medication normally taken (notably Ritalin and Concerta) is known to have primarily short term effects. Moreover, the focus of the present study was placed on attention processes in adult ADHD. This study was clearly not a clinical trial on the effects of medication. Additionally, we also controlled for this medication effect in all statistical analyses. Given that little is known about the long-term effects of medication on neuropsychological performance, future studies should explore this potential influence. To control for the effects of medication on attention, it may be advantageous to use a longer wash out period especially when longer-acting psychoactive medication is taken.

A third issue pertains to the procedures taken during the diagnostic workup. More specifically, this concerns the psychometric properties of the diag- 
nostic interviews and self-report rating scales. The procedures used in the present study were identical to those described in the literature (e.g., Barkley et al., 2001). However, the psychometric properties of these instruments have not yet been investigated with a Dutch speaking population. Nonetheless, they have been shown to be adequate in the USA (Epstein \& Kollins, 2006).

\section{Conclusions}

In summary, the results of this study show that the adults with ADHD only and with comorbidity display a specific deficit in sustained attention compared with nonADHD controls. It pertains to specific regulatory problems in maintaining a consistent level of performance, suggesting a less optimal effort allocation in adult ADHD. The ADHD adults with comorbidity show additional deficits on the focused attention task (i.e., lower accuracy), a finding that was specific to this group, suggesting higher vulnerability to (complex) controlled processing demands and difficulty in inhibiting foils. These findings provide evidence for a specific deficit in sustained attention control, irrespective of comorbidity, rather than a deficient response inhibition as has been noted by Castellanos \& Tannock (2002) in adults with ADHD.

\section{Acknowledgement}

The authors wish to thank Jurgen Cornelis and Ben Hirsch for their clinical expertise and cooperation in the ADHD multidisciplinary team. 


\section{References}

Althaus, M., De Sonneville, L. M. J., Minderaa, R. B., Hensen, L. G. N., \& Til, H. B. (1996a). Information processing and aspects of visual attention in children with the DSM-III-R diagnosis "Pervasive developmental disorder not otherwise specified (PDDNOS)": I. Focused and divided attention. Child Neuropsychology, 2, 17-29.

Althaus, M., De Sonneville, L. M. J., Minderaa, R. B., Hensen, L. G. N., \& Til, H. B. (1996b). Information processing and aspects of visual attention in children with the DSM-III-R diagnosis "Pervasive developmental disorder not otherwise specified (PDDNOS)": ॥. Sustained attention. Child Neuropsychology, 2, 30-38.

American Psychiatric Association. (1994). Diagnostic and statistical manual of mental disorders (4th ed.). Washington DC: American Psychiatric Association.

Barkley, R. A. (1998). Attention-Deficit/ Hyperactivity Disorder: A handbook for diagnosis and treatment (2nd ed.). New York: The Guilford Press.

Barkley, R. A., Murphy, K., \& Kwasnik, D. (1996). Psychological adjustment and adaptive impairments in young adults with ADHD. Journal of Attention Disorders, 1 (1), 41-54.

Barkley, R. A., Murphy, K. R., \& Bush, T. (2001). Time perception and reproduction in young adults with Attention Deficit Hyperactivity Disorder. Neuropsychology, 15(3), 351-360.

Biederman, J., Faraone, S. V., Taylor, A., Sienna, M., Williamson, S., \& Fine, C. (1998). Diagnostic continuity between child and adolescent ADHD: Findings from a longitudinal clinical sample. Journal of the American Academy Child and Adolescence Psychiatry, 37(30), 305-313.

Biederman, J., Mick, E., \& Faraone, S. V. (2000). Age-dependant decline of attention deficit/hyperactivity disorder: Impact of definition of remission. American Journal of Psychiatry, 157, 816-818.

Biederman, J., Newcorn, J., \& Sprich, S. (1991). Comorbidity of Attention Deficit Hyperactivity Disorder with Conduct, Depressive, Anxiety, and other Disorders. American Journal of Psychiatry, 148(5), 564-577.

Börger, N., Van der Meere, J., Ronner, A., Alberts, E., Geuze, R., \& Bogte, H. (1999). Heart rate variability and sustained attention in ADHD children. Journal of Abnormal Child Psychology, 27(1), 25-33.

Brunnekreef, J. A. (2007). Information processing and problem behavior in preadolescents. Enschede: PrintParnters Ipskamp

Castellanos, F. X., \& Tannock, R. (2002). Neuroscience of attention-deficit hyperactivity disorder: the search for endophenotypes. Nature Reviews Neuroscience, 3, 617-628.

Cohen, J. (1988). Statistical power analysis for the behavioral sciences. (2nd ed.). Hillsdale, New Jersey: Erlbaum.

Corkum, P. V., \& Siegel, L. S. (1993). Is the Continuous performance task a valuable research tool for use with children with Attention-Deficit Hyperactivity Disorder? Journal of Child Psychology and Allied Disciplines, 34 (7), 1217-1239.

De Bie, S. E. (1987). Standaardvragen 1987: Voorstellen voor uniformering van vraagstelllingen naar achtergrondkenmerken en interviews. [Standard questions 1987: Proposal for uniformization of questions regarding background variables and interviews]. Leiden: Leiden University Press.

De Sonneville, L. M. J. (1999). Amsterdam Neuropsychological Tasks: A computer-aided assessment program. In B. P. L. M. D. Brinker, P. J. Beek, A. N. Brand, S. J. Maarse \& L. 
J. M. Mulder (Eds.), Cognitive Ergonomics, Clinical assessment and Computer-Assisted Learning: Computers in Psychology. (pp. 187-203). Lisse, the Netherlands: Swets \& Zeitlinger.

De Sonneville L. M. J. (2005). Amsterdamse neuropsychologische taken: Wetenschappelijke en klinische toepassingen. [Amsterdam neuropsychological tasks: Scientific and clinical applications]. Tijdschrift voor Neuropsychologie, 0, 27-41. Dutch.

De Sonneville, L. M. J., Njiokiktjen, C., \& Bos, H. (1994). Methylphenidate and information processing: I. Differentiation between responders and nonresponders: II. Efficacy in responders. Journal of Clinical and Experimental Neuropsychology, 16, 877-897.

Epstein, J. N., Conners, C. K., Sitarenios, G., \& Erhardt, D. (1998). Continuous performance test results of adults with Attention Deficit Hyperactivity Disorder. The Clinical Neuropsychologist, 12, 155-168.

Epstein, J. N., Erkanli, A., Conners, C. K., Klaric, J., Costello, J. E., \& Angold, A. (2003). Relations between continuous performance test performance measures and ADHD behaviors. Journal of Abnormal Child Psychology, 31 (5), 543-554.

Epstein, J. N., Johnson, D. E., Varia, I. M., \& Conners, C. K. (2001). Neuropsychological assessment of response inhibition in adults with ADHD. Journal of Clinical and Experimental Neuropsychology, 23, 362-371.

Epstein, J. N., \& Kollins, S. H. (2006). Psychometric properties of an adult ADHD diagnostic interview. Journal of Attention Disorders, 9(3), 504-514.

Günther T., Herpertz-Dahlmann B., Konrad, K. (2005). Reliabilitat von Aufmerksamkeits- und verbalen Gedachtnistests bei gesunden Kindern und Jugendlichen-Implikationen fur die klinische Praxis. [Reliability of attention and verbal memory tests with normal children and adolescents - clinical implications]. Zeitschrift für Kinder- und Jugendpsychiatrie und Psychotherapie, 33, 169-179. German.

Hanisch, C., Konrad, K., Günther, T., \& Herpertz-Dahlmann, B. (2004). Age-dependent neuropsychological deficits and effects of methylphenidate in children with attention-deficit/hyperactivity disorder: A comparison of pre- and grade-school children. Journal of Neural Transmission, 111, 865-881.

Hervey, A. S., Epstein, J. N., \& Curry, J. F. (2004). Neuropsychology of adults with AttentionDeficit/Hyperactivity Disorder: A meta-analytic review. Neuropsychology, 18(3), 485503.

Kalff, A. C., De Sonneville, L. M., Hurks, P. P., Hendriksen, J. G., Kroes, M., Feron, F. J., et al. (2005). Speed, speed variability, and accuracy of information processing in 5 to 6 year- old children at risk of ADHD. Journal of the International Neuropsychological Society, $11(2), 173-183$.

Konrad, K., Günther, T., Hanisch, C., \& Herpertz-Dahlmann, B. (2004). Differential aspects of methylphenidate on attention functions in children with attention-deficit/hyperactivity disorder. Journal of the American Academy Child and Adolescent Psychiatry, 43(2), 191-198.

Kuntsi, J., Oosterlaan, J., \& Stevenson, J. (2001). Psychological mechanisms in hyperactivity: I respons inhibition deficit, working memory impairment, delay aversion, or something else? Journal of Child Psychology and Psychiatry, 42, 199-210.

Levin, E. D., Conners, C. K., Silva, D., Canu, W., \& March, J. (2001). Effects of chronic nicotine and methylphenidate in adults with attention-deficit/hyperactivity disorder. Experimental Clinical Psychopharmacology, 9(1), 83-90. 
Luteijn, F., \& van der Ploeg, F. A. (1983). Handleiding Groninger Intelligentietest (GIT) [Manual Groningen Intelligence Test]. Lisse, Netherlands: Swets \& Zeitlinger.

Manuzza, S., \& Klein, R. G. (2000). Long-term prognosis in attention-deficit/ hyperactivity disorder. Child and Adolescent Psychiatric Clinics of North America, 9(3), 71 1-726.

Marks, D. J., Newcorn, J. H., \& Halperin, J. M. (2001). Comorbidity in Adults with AttentionDeficit/Hyperactivity Disorder. Annals of New York Academy of Sciences, 931, 216238.

Mirsky, A. F., Pascualvaca, D. M., Duncan, C. C., French, L. M. (1999) A model of attention and its relation to ADHD. Mental Retardation and Developmental Disabilities Research Reviews, 5, 169-176.

Murphy, K., Barkley, R. A., \& Bush, T. (2001). Executive functioning and olfactory identification in young adults with Attention Deficit-Hyperactivity Disorder. Neuropsychology, $15,211-220$.

Nigg, J. T. (2001). Is ADHD a Disinhibitory Disorder? Psychological Bulletin, 127(5), 571-598.

Ossman, J. M., \& Mulligan, N. W. (2003). Inhibition and attention deficit hyperactivity disorder in adults. American Journal of Psychology, $116(1), 35-50$.

Parasuraman, R. (1998). The attentive brain: Issues and prospects. In R. Parasuraman (Ed.), The Attentive Brain. (pp. 3-15). Cambridge, MA: MIT Press.

Pennington, B. F., \& Ozonoff, S. (1996). Executive functions and developmental psychopathology. Journal of Child Psychology and Psychiatry, 37, 51-87.

Pierce, C. A., Block, R. A., \& Aguinis, H. (2004). Cautionary note on reporting eta-squared values from multifactor ANOVA designs. Educational and Psychological Measurement, $64(6), 916-924$.

Rapport, L. J., Van Voorhis, A., Tzelepis, A., \& Friedman, S. R. (2001). Executive functioning in adult attention-deficit hyperactivity disorder. The Clinical Neuropsychologist, 15, 479491.

Sergeant, J. A., Geurts, H., \& Oosterlaan, J. (2002). How specific is a deficit of executive functioning for Attention-Deficit/Hyperactivity Disorder? Behavioral Brain Research, 130, 3-28.

Slaats-Willemse, D. S., Swaab-Barneveld, H., De Sonneville, L., Buitelaar, J. (2005). Familial clustering of executive functioning in affected sibling pair families with ADHD. Journal of the American Academy for Child and Adolescent Psychiatry, 44, 385-391.

Swaab-Barneveld, H., De Sonnevile, L. M. J., Cohen-Kettenis, P., Gielen, A., Buitelaar, J., \& Van England, H. (2000). Visual sustained attention in a child psychiatric population. Journal of the American Academy of Child and Adolescent Psychiatry, 39(5), 651659.

Tannock, R. (2003). Neuropsychology of attention disorders. In S. J. Segalowitz \& I. Rapin (Eds.), Handbook of Neuropsychology (Vol. 8, pp. 753-784). Amsterdam, Netherlands: Elsevier Science B.V.

Van der Meere, J., \& Sergeant, J. (1988). Controlled processing and vigilance in hyperactivity: Time will tell. Journal of Abnormal Child Psychology, 16(6), 641-655.

Van der Meere, J., Wekking, E., \& Sergeant, J. A. (1991). Sustained attention and pervasive hyperactivity. Journal of Child Psychology and Psychiatry and Allied Disciplines, 32. 275-284. 


\section{6 | Chapter 3}

Walker, A. J., Shores, E. A., Trollor, J. N., Lee, T., \& Sachdev, P. S. (2000). Neuropsychological functioning of adults with attention deficit hyperactivity disorder. Journal of Clinical and Experimental Neuropsychology, 22, 115-124.

Ward, M. F., Wender, P. H., \& Reimherr, F. W. (1993). The Wender utah rating scale: An aid in the retrospective diagnosis of childhood attention deficit hyperactivity disorder. American Journal of Psychiatry, 150(6), 885-891.

Weiss, G., \& Trokenberg-Hechtman, L. (1993). Hyperactive children grown up. New York: The Guilford Press.

Willcutt, E. G., Pennington, B. F., Olson, R. K., Chhabildas, N., \& Hulslander, J. (2005). Neuropsychological analyses of comorbidity between reading disability and attention deficit hyperactivity disorder: In search of the common deficit. Developmental Neuropsychology, 27(1), 35-78.

Woods, S. P., Lovejoy, D. W., \& Ball, J. D. (2002). Neuropsychological characteristics of adults with ADHD: A comprehensive review of initial studies. The Clinical Neuropsychologist, $16(1), 12-34$. 


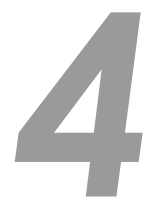

\section{Verbal learning and organisational strategies in adults with ADHD: ADHD subtypes and the impact of comorbidity}




\section{Abstract}

This study examined verbal learning, organisational strategies and the role of comorbidity in two subtypes of adult ADHD. Ninety-eight adults with ADHD combined type (ADHD-C), 37 adults with ADHD inattentive type (ADHD-IA), and 119 healthy controls were compared while performing an auditory verbal learning task. Results showed that, regardless of comorbidity, the ADHD-C group exhibited deficits in verbal working memory, learning, retention, and delayed recall relative to healthy controls. The ADHD-IA group performed better than the ADHD-C group but poorer than the controls. No group differences in serial and subjective organisational strategies emerged. However, use of such strategies did predict verbal learning performance. These findings support the hypothesis that ADHD is a continuum involving distinct subtypes which can be identified by verbal memory impairments. They further suggest that deficient inhibition plays a role in memory impairments in the ADHD-C subtype. 


\section{Introduction}

Attention Deficit Hyperactivity Disorder (ADHD) is one of the most prevalent neurodevelopmental disorders that become manifest in childhood (APA, 2000). Moreover, it persists into adulthood in a substantial percentage of the individuals affected (Manuzza, Klein, \& Moulton, 2003). Many individuals who have been diagnosed with ADHD in adulthood experience problems at work related to cognitive functioning, namely problems with memory, organisation, and concentration (Nadeau, 1995; Quinlan \& Brown, 2003; Weiss \& Murray, 2003). As a consequence many adults with ADHD seek sup-port in health care settings for psychological evaluation.

Neuropsychological studies of adults with ADHD using word-list learning tasks have revealed deficits in verbal learning and aspects of memory, such as encoding and retrieval (Downey, Stelson, Pomerleau, \& Giordani, 1997; Hervey, Epstein, \& Curry, 2004; Holdnack et al., 1995; Lovejoy et al., 1999; Roth et al., 2004; Seidman, Biederman, Weber, Hatch, \& Faraone, 1998; Walker, Shores, Trollor, Lee, \& Sachdev, 2000; Woods, Lovejoy, \& Ball, 2002). These memory impairments are related to deficits in executive functions (EF) that include verbal working memory as an important endophenotype of ADHD symptomatology (Barkley, 1998; Castellanos \& Tannock, 2002; Nigg, Butler, Huang-Pollock, \& Henderson, 2002).

Attempts to delineate the cognitive mechanisms that account for these verbal memory dysfunctions have focused on the performance of verbal learning tasks by ADHD subjects and their use of organised, deliberate rehearsal strategies that involve executive control. However, these studies are limited in number (see review by Hervey et al., 2004) and scope. The present paper presents the findings of the relationship between verbal learning and organisational strategies in ADHD adults in greater detail than ever before. This broadening of scope has been achieved in three important ways.

First of all, unlike previous studies, this study assessed the relationship between verbal learning and organisational strategies in two different ADHD subtypes, as well as the usual control group of healthy subjects. This has made a sound comparison between ADHD subtypes possible for the first time. Secondly, two different types of organisational strategy were selected for examination in this study, namely the passive strategy indicated by serial clustering and the unstructured active strategy indicated by subjective clustering. This has made it possible to assess to what extent differences in verbal learning are due to differences in strategies used by the ADHD subtypes. Thirdly, this study explicitly investigated the impact of comorbidity on memory deficits and all subjects were controlled for interaction with comorbidity.

As a consequence of these three focal points, this study is the first to provide key insights into potential differences in verbal learning and memory between ADHD subtypes, as well as an improved understanding of the potential differences in organisational strategies used, and the effect comorbidity might 
have on verbal learning. The findings of this study will improve our knowledge of the aetiological differences between ADHD subtypes, and contribute to testing the hypothesis that ADHD represents a dimension trait and therefore cannot be regarded as a category (Barkley, 1998; Levy, Hay, McStephen, Wood, \& Waldman, 1997). Below, the relation of these three focal points to previous research is considered before the aims and hypotheses of the current study are presented.

The first topic to discuss is the lack of previous studies to directly investigate verbal memory deficits and the use of organisational strategies with regard to the various subtypes of adult ADHD (i.e., combined, predominantly inattentive, predominantly hyperactive-impulsive; APA, 2000). At present, however, these subtypes are regarded as distinct and valid entities associated with different neuropsychological correlates (Castellanos \& Tannock, 2002) and further research into the differences between them is warranted. Some of the studies conducted previously have indicated the presence of cognitive deficits, such as working memory and verbal learning deficits, in the ADHD combined type (Dinn, Robbins, \& Harris, 2001; Gansler et al., 1998; Murphy, Barkley, \& Bush, 2002). Other studies have mainly associated cognitive deficits, such as executive control and working memory, with inattention (disorganisation) symptoms, indicating cognitive impairment in the inattentive subtype (Gansler et al., 1998; Murphy et al., 2002; Nigg et al., 2005). Yet, despite the fact that they do address specific ADHD subtypes, these studies do not address the question of potential differences in verbal learning and memory, or in organisational strategies, between subtypes. The current study made an important contribution in this respect by investigating two ADHD subtypes, namely ADHD combined type and the ADHD inattentive type, as well as the usual control group of healthy subjects with regard to performance of the auditory verbal learning task

Regarding our second point, three of the previous studies into the relationship between verbal memory dysfunctions and organisational strategies found that, when compared to healthy control subjects, adults with ADHD used a predominantly passive memory strategy indicated by serial clustering (i.e., the number of words recalled in the same order as originally presented to them). The healthy control subjects, on the other hand, used an active strategy which is indicated by semantic clustering (i.e., the number of self-generated words recalled together in the same category regardless of recall order). Additionally, although the ADHD patients performed poorer in semantic clustering, their performance in serial clustering did not differ from that of the healthy control subjects (Holdnack et al., 1995; Roth et al., 2004; Seidman et al., 1998). The relationship between the active strategy indicated by semantic clustering and verbal learning in ADHD patients has been the focus of only one of these previous studies (Roth et al., 2004). It revealed that poor use of semantic organisational strategies did not contribute to poor verbal learning in a general sample of ADHD adults. 
Moreover, only one previous study (Woods et al., 2005) has investigated a more unstructured active strategy known as 'subjective clustering' (i.e., the degree to which pairs of words that are recalled together in one trial are recalled together in the next trial). This study was conducted with adults with methamphetamine dependence (MA), and included a small number of ADHD adults. No differences were observed between the patients and the healthy control subjects with regard to the use of an unstructured active strategy. Unfortunately, the generalisation value of these findings is limited. Nevertheless, due to the fact that recalling more unstructured information in daily life situations is challenging for adults with ADHD, it may be more ecologically valid to examine subjective clustering rather than semantic clustering when studying memory deficits in these cases (see Hervey et al., 2004; Sternberg \& Tulving, 1977). This conclusion is also supported by the fact that in semantic clustering the use of an active but structured organisational strategy is stimulated more explicitly due to fact that the presented word-list consists of semantically related words (Delis, Kramer, Kaplan, \& Ober, 1987; Stuss et al., 1994; Vanderploeg, Schinka, \& Retzlaff, 1994). Therefore, the trials in this study required individuals to perform an auditory verbal learning task consisting of categorically unrelated words and examined the extent to which they used serial clustering and subjective clustering as an organisational strategy.

As yet, therefore, too little is known about the relationship between verbal learning processes and the executive organisational strategies used by ADHD adults. The current study examined this relationship more fully by focusing on the two organisational strategies considered most relevant to an understanding of the aetiology of adult ADHD. Moreover, examining the use of different organisational strategies by different ADHD subtypes has made it possible to assess to what extent differences in verbal learning are due to differences in strategies used by the ADHD subtypes.

Lastly, when it comes to considering whether the memory deficits observed in adult $A D H D$ are due to $A D H D$ or are more likely due to the effects of various comorbidities, the research available until now is inconclusive. ADHD in adults is clearly characterised by high levels of comorbidity, varying from $30 \%$ to $70 \%$, which include antisocial behavior and other personality disorders, as well as substance abuse, anxiety and mood disorders (Barkley, Murphy, \& Kwasnik, 1996; Millstein, Wilens, Biederman, \& Spencer, 1997; Murphy et al., 2002). It is also clear that these disorders have an influence on memory performance when considered on their own (Den Hartog, 2002; Stone, Gabrieli, Stebbins, \& Sullivan, 1998; Verkes et al., 2001).

Although previous studies have controlled for the impact of comorbidity on memory performance in adults with ADHD, the findings generated are inconsistent (see Hervey et al., 2004). Additionally, many studies have failed to account for comorbidity in ADHD subtypes or for the influence of comorbidity on the use of organisational strategies in adults with ADHD. In contrast, the present study has 
controlled for comorbidity in all the subgroups examined, thus directly addressing the question whether memory deficits in adult ADHD subtypes and organisational strategies used by these subtypes, are related to ADHD or to the effects of comorbidity.

Clearly, a lot of ground remains to be covered on the subject of verbal learning deficits in adults with ADHD. It was important, therefore, to address the shortcomings of previous research in order to ensure that new research helps us gain further insight and a greater understanding of adult ADHD and its aetiology. Bearing this in mind, the aims of this study were identified as follows: (a) to investigate verbal learning performance in two ADHD subtypes; (b) to examine whether organisational strategies contribute to verbal learning deficits in these ADHD subtypes; and (c) to examine the impact of comorbidity in these ADHD subtypes.

In order to achieve these aims, adults classified as ADHD-inattentive (ADHD-IA) and ADHD-combined (ADHD-C) were compared with healthy control subjects in terms of their performance on an auditory verbal learning task. We predicted that verbal learning measures would indicate impairments in the ADHD-C and ADHD-IA types. Based on previous findings, we also hypothesised that both subtypes would be impaired with regard to the unstructured active (subjective) organisational strategy and not with regard to the structured passive (serial) clustering strategy. The organisational strategies were also expected to account for the verbal memory impairments in ADHD, thereby supporting the hypothesis of primary executive dysfunction in ADHD. Finally, we hypothesised that the deficits in both ADHD subtypes would be specific for ADHD and not due to the effect of comorbidity. In addition to these aims, we examined whether adult ADHD represents a dimensional trait expressed in qualitative differences on a continuum, with ADHD-C at one end of the spectrum, healthy control subjects at the opposite end, and ADHD-IA between the two extremes (Barkley, 1998; Levy et al., 1997).

\section{Method}

\section{Participants}

The ADHD group consisted of adult patients diagnosed with either ADHD combined type (ADHD-C; $n=98$ ) or ADHD predominantly inattentive type (ADHD-IA; $n=37$ ). Demographic and clinical characteristics for both groups are presented in Table 1. All clinical cases were a series of consecutive ADHD referrals to a specialised outpatient clinic for adults with ADHD at Vijverdal Psychiatric Hospital in Maastricht, the Netherlands. At the time of referral, all individuals exhibited one or more ADHD symptoms and therefore sought the help of a medical specialist who then referred them to this clinic. A multidisciplinary workup for the diagnosis of ADHD corresponding with procedures used in other studies (i.e., Barkley, Murphy, \& Bush, 2001; Barkley, Murphy, DuPaul, \& Bush, 2002) was applied. In brief, the 
diagnostic assessment consisted of a standardised psychiatric workup, including a semistructured diagnostic interview and a clinical interview with the patient. In addition, selfreport rating scales, namely a Dutch version of the ADHD Rating Scale including the 18 DSM-IV criteria (APA, 1994), were completed by the patient. The criterion threshold was as follows: the individual had at least four to five symptoms in each behavioral domain (i.e., inattention versus hyperactivityimpulsivity) in adulthood in agreement with the adult ADHD literature, (Barkley, 1998; Kooij et al., 2004) and six symptoms corresponding to each domain in childhood (APA, 1994). ADHD cases showing comorbidity with other disorders were included in the study. The distribution of one or more comorbid psychiatric disorders for both ADHD subtypes is depicted in Table 2.

The healthy control participants ( $n=119$; see Table 1 for demographic variables) were matched on gender and age to the ADHD patients, and were derived from a sub-sample of the Maastricht Aging Study's (MAAS; $N=1823$ ) baseline database (Bosma, Van Boxtel, Ponds, Houx, \& Jolles, 2003; Jolles, Houx, Van Boxtel, \& Ponds, 1995; Van der Elst, Van Boxtel, Van Breukelen, \& Jolles, 2005). MAAS is a large, prospective, cross-sectional, and longitudinal study on the determinants of cognitive aging at Maastricht University in the Netherlands.

Exclusion criteria for all participants were: (a) the use of stimulants at the time of testing, such as methylphenidate; (b) serious head trauma, including organic syndromes like meningitis; $(c)$ the presence of neurological pathology or medical disease(s), such as epilepsy, dementia, cerebrovascular disease, parkinsonism, malignancies related to the nervous system, and diabetes mellitus; and (d) an IQ score less than 85. Participants taking medication other than stimulants (i.e., antidepressants) were included. All ADHD participants were asked to discontinue their stimulant medication treatment 24 hours prior to the testing. The healthy control sample had a number of additional exclusion criteria: (a) psychopathology, including ADHD and learning disabilities (dyslexia); (b) current chronic psychotropic drug use, such as medication, alcohol, and drugs; and (c) selfreported treatment for psychiatric disorders at the time of testing.

After applying the exclusion criteria, the final sample consisted of 135 outpatients with ADHD and 119 healthy control subjects. Table 1 shows that the three groups were comparable with respect to gender and age. The ADHD-C and ADHD-IA groups had a significantly lower IQ than the healthy control subjects, and the ADHD-C group displayed higher medication usage than the two other groups. In line with the clinical diagnosis, the ADHD-IA group had significantly lower hyperactivity-impulsivity symptoms than the ADHD-C group, and both groups were comparable with respect to their inattention symptoms.

\section{Procedure}

All ADHD patients were administered a routine neuropsychological evaluation including an auditory verbal learning task (AVLT) (according to standard instructions and scoring procedures). Data of the AVLT for the healthy control subjects 
were gathered during a baseline test program of the MAAS study (Jolles et al., 1995; Van Boxtel et al., 1998)). All testing was conducted under the supervision of licensed health care/clinical psychologists and clinicians.

Table 1. Demographic and AVLT performance data for all groups

\begin{tabular}{lccc}
\hline Variable & ADHD-C & ADHD-IA & Control \\
& $(n=98)$ & $(n=37)$ & $(n=119)$ \\
\hline Gender male/female & $74 / 24$ & $22 / 15$ & $81 / 38$ \\
Medication yes/no & $32 / 66$ & $4 / 33$ & $0 / 119 \mathrm{~b}$ \\
Age (M/SD) & $32.7(8.9)$ & $31.9(10.3)$ & $33.9(8.3)$ \\
Range & $19-52$ & $17-57$ & $24-56$ \\
IQa M (SD) & $107.4(11.0)$ & $107.9(12.1)$ & $113.0(10.0)^{\mathrm{c}}$ \\
Range & $85-129$ & $85-129$ & $89-134$ \\
DSM_IA (P) M (SD) & $6.3(2.2)$ & $7.1(2.0)$ & \\
DSM_HI (P) M (SD) & $6.2(2.3)$ & $4.0(3.0)$ d & \\
AVLT Trial 1 M (SD) & $4.7(1.6)$ & $5.6(1.9)$ & $6.1(1.5)^{\mathrm{b}}$ \\
AVLT Delayed recall & & & \\
M (SD) & $8.2(3.1)$ & $9.6(3.3)$ & $10.3(2.5)^{\mathrm{e}}$ \\
AVLT Retention (\%) & & & $86.8(13.8)^{\mathrm{e}}$ \\
M (SD) & $76.6(22.0)$ & $81.4(22.4)$ & \\
\hline
\end{tabular}

Notes. ADHD-C = Attention Deficit Hyperactivity Disorder, combined subtype. ADHD-IA = Attention Deficit Hyperactivity Disorder, inattentive subtype. DSM_IA (P) = present total inattention symptoms (max. =9). DSM_HI $(P)$ = present total hyperactivity-impulsivity symptoms (max. =9). Associations between ordinal and/or interval variables (group membership vs. age, IQ, DSM_IA, and DSM_HI) were measured by AN(C)OVAs. X2 tests were used to measure the associations between nominal (gender, medication) and ordinal variables (groups). alQ was measured with the shortened form of the Dutch Groningen Intelligence Test (GIT) (Luteijn \& van der Ploeg, 1983). ' ${ }^{\text {ADHD-C }}$ group was significantly different than the two other groups. ${ }^{A}$ ADHD-C and ADHD-IA groups were significantly different than the control subjects. ${ }^{A}$ ADHD-C group was significantly different than ADHD-IA group. ${ }^{A}$ ADHD-C group was significantly different than control group.

\section{Measurements}

The Auditory Verbal Learning Task (AVLT; Brand \& Jolles, 1985; Rey, 1964; Van der Elst et al., 2005), a Dutch modification of the Rey Auditory Verbal Learning Test, was used to measure verbal learning and memory. Fifteen words were auditorily and sequentially presented. Participants were then asked to recall as many words as possible in any order. This procedure was repeated five times. After 20 minutes, participants were again asked to reproduce as many of the words as possible. This was a measure of delayed recall. Considerable normative data have been gathered in related studies (Houx, Vreeling, \& Jolles, 1991; Van der Elst et al., 2005). The following dependent variables were analysed: Trial 1 as a measure of working memory or shortterm memory (Schmidt, 1996; Van der Elst 
et al., 2005); Trial 1, 2, 3, 4, 5 as an index of the learning rate curve; delayed recall; and retention score. The adapted form of the serial clustering measure was calculated (Bouma, Mulder, \& Lindeboom, 1996; Kramer, Delis, Kaplan, O'Donnell, \& Prifitera, 1997) and was included as a measure of simple strategy

Table 2. Distribution of psychopathology other than ADHD for both ADHD groups

\begin{tabular}{|c|c|c|}
\hline DSM-IV disorders & $\begin{array}{l}\text { ADHD-C } \\
(n=54)\end{array}$ & $\begin{array}{l}\text { ADHD-IA } \\
(n=18)\end{array}$ \\
\hline PD NOS & 13 & 4 \\
\hline Borderline PD & 5 & 1 \\
\hline Antisocial PD & 1 & \\
\hline Mood disorders & 5 & 2 \\
\hline Anxiety disorders & 3 & 2 \\
\hline Substance-related disorders & 5 & 1 \\
\hline Learning disorder (dyslexia) & & 1 \\
\hline Adaptation disorder & 1 & 2 \\
\hline Autism spectrum disorder & 1 & \\
\hline Tourette's syndrome & 1 & \\
\hline Oppositional Defiant Disorder & 1 & \\
\hline Mood disorders (or in remission) \& anxiety disorders & 2 & \\
\hline Mood disorders \& PD NOS/ borderline PD & 4 & 3 \\
\hline Mood disorders \& substance-related disorder & 2 & \\
\hline Mood Disorder \& eating disorder NOS & 1 & \\
\hline Anxiety Disorders \& PD NOS & 1 & \\
\hline Substance-related disorder \& PD NOS & 2 & 2 \\
\hline Impulse control disorder \& PD NOS & 2 & \\
\hline PD NOS \& eating disorder & 1 & \\
\hline $\begin{array}{l}\text { Mood disorders \& PD NOS/antisocial \& substance-related } \\
\text { disorders }\end{array}$ & 3 & \\
\hline
\end{tabular}

Note. PD NOS = personality disorder, not otherwise specified.

use. The serial clustering index per trial was calculated by dividing the total number of observed serial clusters (SC) during trial i by the total number of expected serial clusters by chance. Finally, to measure subjective clustering, the adjusted form of the Pair Frequency (PF) was calculated per pair of trials as an index of complex strategy use in free recall (for more detailed information see Bouma et al., 1996; Schmidt, 1996; Sternberg \& Tulving, 1977). This PF measure was considered to be an index of the degree to which pairs of words that are recalled 
together during one trial are recalled together during the next trial. The word order in the pair was not important and was also considered to be bidirectional. To obtain a clear measure of subjective clustering, serial clusters were excluded from this calculation.

Statistical analysis

All data were first screened for violations of the assumptions of General Linear Models analyses (GLM). Because not all data were normally distributed (i.e., retention), non-parametric Kruskall-Wallis tests were used to verify our parametric results. These tests did verify our results and therefore parametrics are described. Two-tailed $P$ values of .05 or lower were considered statistically significant.

Group differences in the verbal learning and organisational strategies measures were tested with separate GLM univariate analyses. GLM repeated measures designs were utilised to detect significant differences in verbal learning, and serial and subjective clustering as a function of trials. Greenhouse-Geisser correction probabilities were used to deal with unequal sample sizes and violations of Mauchly's Test of Sphericity. Post-hoc analyses were performed with a Bonferroni correction for multiple comparisons. Polynomial contrasts were used to examine the linear effect across the three group classification (i.e., to examine whether the groups could be arranged on a continuum, with ADHD-C at one extreme, healthy control subjects at the other extreme, and ADHD-IA in between). Medication usage was included as a covariate in all analyses. In addition, all analyses were repeated with $I Q$ as a second covariate, as this variable accounted for at least some variance in the verbal memory scores and some authors assert that controlling for this intermediating factor makes for a stronger case when differences still exist (Murphy et al., 2001; Sergeant, Geurts, \& Oosterlaan, 2002).

We also investigated whether performance on the AVLT measures between the subgroups differed as a function of comorbid psychiatric disorders. The total sample (ADHD-C, ADHD-IA, control subjects) was divided into two groups, namely those adults with comorbidity and those without comorbidity. This nominal variable was included in all analyses as a between-subject factor in order to determine whether there was an interaction between the subgroups and psychiatric comorbidity.

Pearson correlation analyses were used to examine the relationships between the ADHD symptoms (i.e., inattention vs. hyperactivity-impulsivity) and the various AVLT outcome measures in the ADHD sample. They were also used to explore whether the assumptions for regression analyses were met (Kleinbaum, Kupper, Muller, \& Nizam, 1998). According to Cohen (1977), correlations of .30 to .50 are considered moderate in size and those above .50 are regarded as large.

Multiple linear regression analyses were conducted to investigate: (a) the relationship between the verbal learning measures and organisational strategies; and (b) interactions between organisational strategies and ADHD behavioral 
symptoms in the ADHD sample. The regression models were fitted using a stepdown hierarchical procedure for the AVLT measures. Initially, all predictors, interactions, and covariates (serial clustering, subjective clustering, inattention symptoms, hyperactivity-impulsivity symptoms, serial $\times$ number criteria of DSM_IA, serial $\times$ number criteria of DSM_HI, subjective $\times$ number criteria of DSM_IA, subjective $\times$ number criteria of DSM_HI, medication, IQ, and comorbidity) were entered into the regres-sion models simultaneously using the enter method. Interactions between the separate organisational strategies and inattention and hyperactivity symptoms were included in the start model to examine the unique contribution of the strategies that could not be accounted for by the behavioral symptoms. Non-significant interactions and predictors were then removed from the model, but no predictor was removed from the model as long as it was also included in a higher order term in the model.

\section{Results}

Group differences in verbal learning measures

GLM univariate analyses revealed significant main effects of group for the AVLT Trial $1\left(F(2,236)=11.31, p<.001 ; \eta p^{2}=.08\right)$, delayed recall $(F(2,227)=10.40, p<$ $\left..001 ; \eta p^{2}=.08\right)$, and retention $\left(F(2,219)=6.92, p=.001 ; \eta p^{2}=.06\right)$. Post-hoc analyses indicated that the ADHD-C group performed significantly poorer on these three measures when compared to the healthy control subjects, and the ADHD$C$ adults tended to perform poorer than subjects with ADHD-IA regarding the AVLT Trial 1 ( $p=.056$ ) (see Table 1). Results were essentially the same after correcting for $I Q$. The polynomial contrasts were significant $(p<.001)$, showing a linear effect for the AVLT trial 1, delayed recall and retention in which the healthy control subjects performed the best, ADHD-C adults performed the poorest, and the ADHD-IA adults performed somewhere between these two groups.

A 5 (trial) $\times 3$ (groups) $\times 2$ (comorbidity) repeated measures analysis revealed a significant trial effect $\left(F(4,832)=689.25, p<.001 ; \eta_{p}^{2}=.76\right)$ and $a$ significant main effect of group $\left(F(2,208)=4.20, p=.016 ; \eta_{p}^{2}=.03\right)$. All three groups showed an increase in performance over the five trials. Over the course of the trials, the ADHD-C patients performed poorer than the healthy control subjects $(p<.05)$ (see Figure 1 left). No subtype differences emerged. The polynomial contrast was significant ( $p=.015)$, showing a linear effect for verbal learning, with the healthy control subjects showing the highest overall score and the ADHD-C adults the lowest. Results with correction for IQ attenuated the main effect for groups ( $p=$.082), revealing no post-hoc group differences.

There were no significant main effects or interactions for psychiatric comorbidity ( $p>.10$ for all analyses) for any of the AVLT measures. 


\section{Group differences in organisational strategies}

A 5 (trial by serial clustering) $\times 3$ (groups) $\times 2$ (comorbidity) repeated measures design revealed a significant effect for trial, $F(4,808)=3.19, p=.015 ; \eta_{p}^{2}=.01$, and a significant trial by group interaction, $F(8,808)=2.60, p=.010 ; \eta_{p}^{2}=.02$. However, no main effect of group $\left(F(2,202)=1.91, p=.150 ; \eta_{p}^{2}=.01\right)$ was found with respect to serial clustering. Both ADHD-C and ADHD-IA groups used gradually more serial clustering from trial 3 on, while the healthy control subjects showed a decrease in trial 3 (see Figure 1 middle). The polynomial contrast was not significant, showing no linear effect. Results were essentially the same once corrected for IQ.

With regard to subjective clustering, one ADHD-C and one ADHD-IA participant were excluded from the analyses due to extreme value (within-subjects standard deviations $>3$ times interquartile range from the median). A 4 (trial pair) $\times 3$ (groups) $\times 2$ (comorbidity) repeated measures design revealed a significant effect for trial pair, $F(3,624)=12.68, p<.001 ; \eta p^{2}=.05$, and a trend towards a main effect of group, $F(2,208)=2.76, p=.065 ; \eta_{p}^{2}=.02$, but no significant trial pair by group interaction ( $p=.669$ ) on subjective clustering (see Figure 1 right). Overall, the individuals displayed an increase in subjective clustering over trial pairs mainly from trial pair $1(M=.08)$ to trial pair $2(M=.54)$. Post-hoc analyses indicated that the ADHD-C patients showed less subjective clustering than the healthy control subjects $(p=.056)$. The polynomial contrast was significant $(p=$ .017), demonstrating a linear effect for subjective clustering, with the healthy control subjects showing the highest clustering, the ADHD-C adults showing the lowest clustering, and the ADHD-IA adults performing somewhere in between. Results were the same once corrected for $I Q$, but attenuated the main effect for group ( $p=.128$ ) revealing no post-hoc group differences. There were no significant main effects or interactions for psychiatric comorbidity $(p>.10$ for all analyses) for any of the organisational measures.

\section{Contribution of organisational strategies}

The bivariate associations between AVLT measures and ADHD symptoms for the total ADHD sample are shown in Table 3. Significant correlations between hyperactivity-impulsivity symptoms and AVLT performance measures (Trial 1, total score trial 1-5) were found. These correlations were low, and better performance on the AVLT measures was associated with lower hyperactivity-impulsivity symptoms. No significant correlations were found between the ADHD symptoms and delayed recall, retention, and between symptoms and the clustering strategies (serial versus subjective).

The final regression models (after step-down hierarchical removal of nonsignificant interactions, covariates and predictors) for the total ADHD sample are depicted in Table 4. The model indicated that serial clustering and hyperactivityimpulsivity symptoms contributed unique variance to performance on AVLT Trial 1 
Verbal learning in adults with ADHD | 89
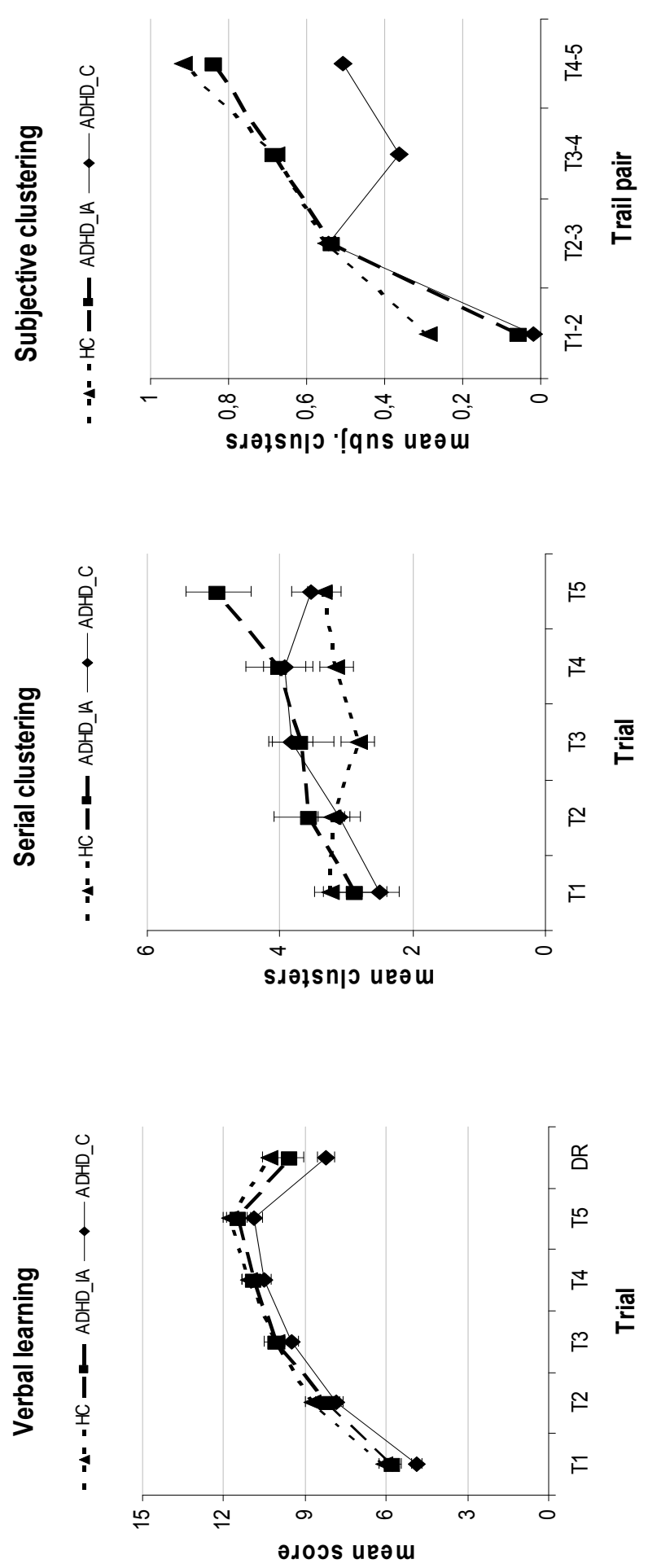

点 ᄃ 늘 $\stackrel{4}{\square}$ $\rightleftarrows$ $\stackrel{0}{\rightleftarrows} \leq$ ○. 옹 ن) 을 을

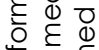
के 흥 워 $\stackrel{0}{0}$ 过 号 $\overline{\bar{O}} \overline{\bar{\alpha}}$

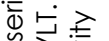

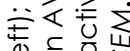
$\stackrel{=}{=}$ O $\overline{\overline{0}}$ 흥 응 을 흠

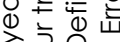
ㅎํㅇํำ ब

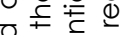

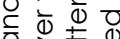
约全全 을 \& $11 \frac{0}{0}$ (1) 추웅

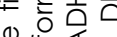
继 $<0$ 뇨욜 $\sum_{0}^{0}$ O)

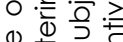
(1) 등믄항 हो ए 하웡 ब.

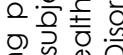
엥

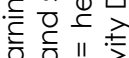
Q $\overline{0} \overline{0}$ 工 히을 웅 $>$ हᄐ은조 ํㅣㅁ 
(see Table 4). Both serial and subjective organisational strategies contributed unique variance to performance on verbal learning and delayed recall (see also Table 4), but not on the retention measure. There were no significant interactions between the ADHD symptoms and the organisational strategies.

Table 3. Correlation matrix between AVLT measures and ADHD symptoms for ADHD Sample

\begin{tabular}{lccllllll}
\hline Variable & 1 & 2 & 3 & 4 & 5 & 6 & 7 & 8 \\
\hline 1. AVLT Trial 1 & - & $.65^{* *}$ & $.45^{* *}$ & $.21^{* *}$ & $.35^{* *}$ & .11 & .03 & $-.24^{*}$ \\
2. AVLT Trial 1-5 & & - & $.70^{* *}$ & $.32^{* *}$ & $.36^{* *}$ & $.23^{* *}$ & -.04 & $-.20^{*}$ \\
3. AVLT Delayed recall & & & - & $.77^{* *}$ & $.22^{*}$ & $.19^{*}$ & -.11 & -.18 \\
4. AVLT Retention (\%) & & & & - & .08 & .14 & -.14 & -.19 \\
5. AVLT Serial cl. & & & & & - & $-.27^{* *}$ & .11 & -.03 \\
6. AVLT Subject. cl. & & & & & & - & -.00 & -.15 \\
7. DSM_IA & & & & & & - & $.27^{* *}$ \\
8. DSM_HI & & & & & & & - \\
\hline
\end{tabular}

Note. AVLT = Auditory Verbal Learning Task. AVLT Serial cl. = total serial clustering over five trials. AVLT Subject. $c l .=$ total subjective clustering over four trial pairs. DSM_IA = number of inattention symptoms. DSM_HI = number of hyperactivity-impulsivity symptoms. ${ }^{*} p<.05 .{ }^{* *} p<.01$.

Table 4. Final linear regression models for verbal learning measures for ADHD sample

\begin{tabular}{|c|c|c|c|c|c|c|c|}
\hline Score & Variable & $\beta$ & SE & $t$ & Standardised $\beta$ & $\mathrm{R}^{2}$ & $p$ \\
\hline \multirow{3}{*}{ AVLT Trial 1} & (constant) & 5.107 & .496 & 10.28 & \multirow{2}{*}{.198} & & .000 \\
\hline & AVLT Serial. cl. & .040 & .019 & 2.06 & & & 0.42 \\
\hline & DSM_HI & -.151 & .060 & -2.45 & -.239 & .82 & .014 \\
\hline \multirow{3}{*}{$\begin{array}{l}\text { AVLT Trial } \\
1-5\end{array}$} & (constant) & 29.292 & 2.053 & 14.27 & & & .000 \\
\hline & $\begin{array}{l}\text { AVLI Serial. } \\
\text { cl. }\end{array}$ & .596 & .101 & 5.89 & .473 & & .000 \\
\hline & $\begin{array}{l}\text { AVLT Subject. } \\
\text { cl. }\end{array}$ & 1.547 & .345 & 4.48 & .360 & .248 & .000 \\
\hline \multirow{3}{*}{$\begin{array}{l}\text { AVLT } \\
\text { Delayed } \\
\text { recall }\end{array}$} & (constant) & 6.174 & .676 & 9.139 & & & .000 \\
\hline & $\begin{array}{l}\text { AVLI serial. } \\
\text { cl. }\end{array}$ & .111 & .033 & 3.38 & .310 & & .001 \\
\hline & $\begin{array}{l}\text { AVLT Subject. } \\
\text { cl. }\end{array}$ & .352 & .114 & 3.09 & .284 & .110 & .002 \\
\hline
\end{tabular}

Note. AVLT = Auditory Verbal Learning Task. AVLT Serial. cl. = total serial clustering over five trials. DSM_HI = hyperactivity-impulsivity symptoms. AVLT Subject. $\mathrm{cl}$. = total serial clustering over four trial pairs.

\section{Discussion}

The current study required adults with ADHD-C and ADHD-IA subtypes to perform an auditory verbal learning task consisting of categorically unrelated words and examined their use of organisational strategies, as well as the impact of comorbi- 
dity on their performance. Findings suggested that verbal memory deficits in ADHD can be arranged on a continuum, with ADHD-C representing the poorest performance, the healthy control subjects representing the best performance, and the performance by the ADHD-IA subtype placed somewhere in between. These new findings support the hypothesis that there are distinct subtypes of ADHD in adults (APA, 2000; Barkley, 1998; Castellanos \& Tannock, 2002). In addition, it became clear that these findings were not influenced by the effect of comorbidity in the ADHD groups. These findings will be discussed in greater detail below.

Our first aim was to investigate differences in verbal learning and memory between ADHD subtypes. Results showed that, when compared to healthy control subjects, patients with ADHD-C exhibit specific deficits in verbal working memory, verbal learning, and delayed recall. This finding only partially supported our first hypothesis, which also predicted impairments in the ADHD-IA subtype, for no deficits in verbal memory were found for the latter. Previous research examining these differences in subtypes has been rather inconsistent in demonstrating verbal (working memory) impairments in ADHD-IA (Gansler et al., 1998; Murphy et al., 2002; Nigg et al., 2005), although some studies have found similar neuropsychological profiles in adult subtypes (Murphy et al., 2001).

Conversely, our findings of specific verbal learning and memory deficits in the ADHD-C group, which consists of additional hyperactivity-impulsivity symptoms relative to the ADHD-IA group, suggest that a deficient inhibitory control gives rise to the verbal memory deficits. This is likely to be due to the fact that memory performance in ADHD-IA adults was generally between that of the ADHD-C adults and the healthy control subjects. This is in line with the view that ADHD represents a continuum or a dimension, and not a category with strict boundaries (Barkley, 1998; Levy et al., 1997).

Furthermore, the finding that the ADHD-IA individuals performed slightly better than the ADHD-C type individuals supports previous research. This suggests that, in adulthood, different underlying core deficits and mechanisms are involved in both ADHD subtypes (Castellanos \& Tannock, 2002; Diamond, 2005; Gansler et al., 1998). Thus, our interpretation of this data, as suggesting that there is a core deficit in response inhibition in ADHD-C patients, corresponds with recent theoretical models that suggest a core deficit in response inhibition in the ADHD-C type in children (Barkley, 1998; Castellanos \& Tannock, 2002; Nigg et al., 2002).

Our second aim was to examine whether organisational strategies account for deficits in verbal learning and memory in the two ADHD subtypes. We made use of two organisational strategies to uncover the underlying cognitive processes involved, namely a more complex, unstructured active strategy indicated by subjective clustering and a simple, structured passive strategy indicated by serial clustering. Overall, we found no differences between the ADHD subtypes in either of these organisational strategies. On the other hand, our 
examination of interaction between serial clustering and the diagnostic groups indicated that, over the course of the trials, both ADHD subtypes showed higher levels of serial clustering than the healthy control subjects. To the best of our knowledge, this finding is new because previous studies have only focused on a general ADHD (sub)-sample (i.e., Roth et al., 2004; Woods et al., 2005), and not on ADHD subtypes. This finding may imply that ADHD adults, regardless of subtype, favour a predominantly passive kind of organisational strategy by comparison to healthy control subjects. Likewise, with respect to subjective clustering, our results indicated that ADHD-C adults showed a tendency to perform poorer on subjective clustering by comparison to the healthy control subjects, while the ADHD-IA adults performed somewhere between these two groups. This too is a new finding and is also consistent with our expectations. In line with these results, our regression analyses demonstrated that the use of serial clustering during encoding was a significant predictor of verbal working memory performance. Furthermore, both serial and subjective clustering predicted better performance on verbal learning and delayed recall. Our pattern of findings suggests that poor verbal learning performance in adults with ADHD is partially related to the use of organisational strategies and memory organisational strategy selection. This is a new discovery regarding ADHD subtypes.

This present result may indicate that adults with ADHD-C have pro-blems with the controlled information processing strategies involved in subjective clustering but do not have as many problems with the automatic information processing strategies demanded by serial clustering. This pattern was less explicit for ADHD-IA, which may indicate that these patients have less difficulty in automatic processing strategies and may therefore be able to profit more from their use of controlled information processing strategies. We contend that, although these inattentive type adults are capable of using serial clustering, they tend to use a more subjective clustering than the ADHD-C adults and a less subjective clustering than the healthy control subjects.

One way to interpret these findings involves looking at the relationship between working memory and memory performance (Repovs \& Baddeley, 2006). Working memory is a limited-capacity system in which information can be held active over a relatively short period of time. Working memory is needed during the encoding stages and underlies a wide range of cognitive processes (Baddeley, 1986). One can contend that, with an overloaded or deficient verbal working memory and limited attention span, adults with ADHD adopt less demanding memory strategies, which subsequently results in verbal memory deficits as expressed by Trial 1. These disruptions can in turn manifest themselves in behavior, such as forgetfulness in daily activities (APA, 2000).

Therefore, we argue that the source of the verbal memory weakness in the ADHD-C type is likely to be associated with an inefficient capacity of the working memory during the initial learning phase. This means that these adults essentially start off with a lower performance while also experiencing difficulties when com- 
pensating for this deficit. This, in turn, leads to inefficient encoding and delayed recall. The influence of a deficient verbal working memory capacity and the associated limited attention span is compatible with previous theoretical models on working memory (Baddeley, 1986) that suggest a restrained phonological loop (verbal working memory). This explanation also supports the recent theoretical proposition of a core deficit of inhibition and of secondary impairment in (verbal) working memory, which are considered potential cognitive endophenotypes in the ADHD combined type (Barkley, 1998; Castellanos \& Tannock, 2002).

Our final aim was to examine the impact of comorbidity on verbal memory performance in the ADHD subtypes. Based on the use of interaction in our approach, we found that the presence of one or more comorbid psychiatric disorders did not influence the results of any of the verbal memory measures. This finding supplements the limited previous research (Barkley et al., 2001; Murphy et al., 2001) regarding ADHD subtypes. The fact that comorbidity did not influence our findings also supports the validity of recognising ADHD subtypes as distinct disorders which are not an artefact of prevalent comorbid disorders (Barkley, 1998; Millstein et al., 1997).

With the exception of verbal learning, our findings remained consistent after accounting for IQ. Our data do suggest that it is important to control for IQ in order to obtain clear and specific deficits related to ADHD subtypes. Our study also indicates that verbal learning performance is more likely to be dependent on intellectual abilities. A finding supporting previous studies that have also found associations between IQ and cognitive performance in ADHD (Bridgett \& Walker, 2006; Sergeant et al., 2002).

Some limitations to our research require consideration. Primarily, the inclusion of multiple comorbid disorders might have limited our findings. Replication of this study with more circumscribed comorbid conditions in the ADHD subtype samples would reduce this heterogeneity, and allow for a more direct application of the findings to these specific populations. The present study did, however, provide a clear picture of comorbidity in ADHD subtypes in a clinical setting, which makes it representative of, and applicable to, a clinical population. Furthermore, our non-significant findings with respect to organisational strategies might have also been influenced by the small sample size. Finally, we focused on two types of organisational strategies as possible underlying mechanisms for the deficits in verbal learning and memory in adults with ADHD. Studies assessing whether ADHD adults show fewer organisational strategies in other memory and cognitive tasks, such as verbal fluency tasks, would be beneficial to determine the generalisation value of our findings.

However, despite these limitations, the present study adds to the existing literature and theoretical models by providing data on the distinctiveness of verbal learning performance and memory deficits in subtypes of ADHD adults. Therefore, it has contributed by facilitating the evaluation of more specific hypotheses in this cognitive domain in larger samples of individuals with ADHD. 
Our results have also several implications. We have reported that the ADHD-C individuals are most likely to achieve low scores on a verbal learning task. These deficits in verbal learning have been associated with performance on other cognitive functions, including executive functions and inhibiting responses (Hervey et al., 2004). Clearly, adults with the combined ADHD type may exhibit a range of functional impairments due to deficits in their working memory and verbal learning, for example forgetting what was written on their shopping list in the supermarket. As a result, poor verbal learning and working memory capacity may be a cognitive factor that contributes to the academic, work-related, and societal difficulties experienced by adults with ADHD (Faraone et al., 2000).

Furthermore, based on our findings, we contend that providing support or treatment for verbal memory impairments in adults with ADHD-C may help to reduce their functional impairments in daily life. For example, treatments may become more effective by including training programs that focus on enhancing verbal working memory and verbal learning capacity (Klingberg et al., 2005). In addition, teaching these adults how to efficiently use memory strategies (i.e., cueing, clustering, limiting the context of extraneous stimuli, strategy instructions/ plans) may improve their cognitive skills and general psychosocial functioning. 


\section{References}

American Psychiatric Association (2000). Diagnostic and Statistical Manual of Mental Disorders: DSM-IV-TR. Washington, DC: American Psychiatric Press.

American Psychiatric Association (1994). Diagnostic and Statistical Manual of Mental Disorders (4th ed.). Washington DC: American Psychiatric Association.

Baddeley, A. (1986). Working memory. Oxford, England: Clarendon Press.

Barkley, R. A. (1998). Attention-Deficit/ Hyperactivity Disorder: A handbook for diagnosis and treatment (2nd ed.). New York: The Guilford Press.

Barkley, R. A., Murphy, K., \& Kwasnik, D. (1996). Psychological adjustment and adaptive impairments in young adults with ADHD. Journal of Attention Disorders, 1 (1), 41-54.

Barkley, R. A., Murphy, K. R., \& Bush, T. (2001). Time perception and reproduction in young adults with Attention Deficit Hyperactivity Disorder. Neuropsychology, 15(3), 351-360.

Barkley, R. A., Murphy, K. R., DuPaul, G. J., \& Bush, T. (2002). Driving in young adults with attention deficit hyperactivity disorder: Knowledge, performance, adverse outcomes, and the role of executive functioning. Journal of the International Neuropsychological Society, 8, 655-672.

Bosma, H., van Boxtel, M. P. J., Ponds, R. W. H. M., Houx, P. J., \& Jolles, J. (2003). Education and age-related cognitive decline: the contribution of mental workload. Educational Gerontology, 29, 1-9.

Bouma, A., Mulder, J., \& Lindeboom, J. (1996). Neuropsychologische diagnostiek: Handboek. Amsterdam, the Netherlands: Swets \& Zeitlinger.

Brand, N., \& Jolles, J. (1985). Learning and retrieval rate of words presented auditory and visually. Journal of General Psychology, 112, 201-210.

Bridgett, D. J., \& Walker, M. E. (2006). Intellectual functioning in adults with ADHD: A metaanalytic examination of full scale IQ differences between adults with and without ADHD. Psychological Assessment, 18(1), 1-14.

Castellanos, F. X., \& Tannock, R. (2002). Neuroscience of attention-deficit/hyperactivity disorder: the search for endophenotypes. Nature Reviews Neuroscience, 3, 617-628.

Cohen, J. (1977). Statistical power analysis for the behavioral sciences. (revised ed.). New York: Academic Press.

Delis, D. C., Kramer, J. H., Kaplan, E., \& Ober, B. A. (1987). California Verbal Learning Test: Adult version. San Antonio, TX: The Psychological Corporation.

Den Hartog, M. (2002). Cognitive performance in depression: Patterns and determinants. Maastricht, the Netherlands: Neuropsych Publishers.

Diamond, A. (2005). Attention-deficit disorder (attention-deficit/hyperactivity disorder without hyperactivity): A neurobiologically and behaviorally distinct disorder from attention-deficit/hyperactivity disorder (with hyperactivity). Development and Psychopathology, 17, 807-825.

Dinn, W. M., Robbins, N. C., \& Harris, C. L. (2001). Adult Attention-Deficit/Hyperactivity Disorder: Neuropsychological correlates and clinical presentation. Brain and Cognition, 46(1-2), 114-121.

Downey, K. K., Stelson, F. W., Pomerleau, O. F., \& Giordani, B. (1997). Adult Attention Deficit Hyperactivity Disorder: Psychological test profiles in a clinical population. Journal of the Nervous and Mental Disorders, 185, 32-38. 
Faraone, S. V., Biederman, J., Spencer, T., Wilens, T., Seidman, L. J., Mick, E., et al. (2000). Attention-Deficit/Hyperactivity Disorder in adults: An Overview. Biological Psychiatry, 48, 9-20.

Gansler, D. A., Fucetola, R., Krengel, M., Stetson, S., Zimering, R., \& Makary, C. (1998). Are there cognitive subtypes in adult Attention Deficit/Hyperactivity Disorder? The Journal of Nervous and Mental Disease, 186, 776-781.

Hervey, A. S., Epstein, J. N., \& Curry, J. F. (2004). Neuropsychology of adults with AttentionDeficit/Hyperactivity Disorder: A meta-analytic review. Neuropsychology, 18(3), 485503.

Holdnack, J. A., Moberg, P. J., Arnold, S. E., Ruben, C., Gur, R. C., \& Gur, R. E. (1995). Speed of processing and verbal learning deficits in adults diagnosed with Attention Deficit Hyperactivity Disorder. Neuropsychiatry, Neuropsychology, and Behavioral Neurology, 8(4), 282-292.

Houx, P. J., Vreeling, F. W., \& Jolles, J. (1991). Age associated cognitive decline is related to biological life-events. In R. C. Mclachlan, B. Winblad \& H. M. Wisniewski (Eds.), Alzheimer's Disease: Basic mechanisms, diagnosis and therapeutic strategies. (pp. 353-359). Chichester: Wiley.

Hurks, P. P. M., Hendriksen, J. G. M., Vles, J. S. H., Kalff, A. C., Feron, F. J. M., Kroes, M., et al. (2004). Verbal fluency over time as a measure of automatic and co-trolled processing in children with ADHD. Brain and Cognition, 55, 535-544.

Jolles, J., Houx, P. J., Van Boxtel, M. P. J., \& Ponds, R. W. H. M. (1995). The Maastricht Aging Study: Determinants of cognitive aging. Maastricht: Neuropsych Publishers.

Kleinbaum, D. G., Kupper, L. L., Muller, K. E., \& Nizam, A. (1998). Applied regression analysis and other multivariate methods (third ed.). New York: Duxbury Press.

Klingberg, T., Fernell, E., Olesen, P. J., Johnson, M., Gustafsson, P., Dahlström, K., et al. (2005). Computerized training of working memory in children with ADHD- A randomized, controlled trial. Journal of the American Academy Child and Adolescence Psychiatry, 44(2), 177-186.

Kooij, J. J. S., Buitelaar, J. K., van den Oord, E. J., Furer, J. W., Rijnders, C. A. T., \& Hodiamont, P. P. G. (2004). Internal and external validity of Attention-Deficit Hyperactivity Disorder in a population-based sample of adults. Psychological Medicine, 34, 1-1 1.

Kramer, J. H., Delis, D. C., Kaplan, E., O' Donnell, L. O., \& Prifitera, A. (1997). Develop-mental differences in verbal learning. Neuropsychology, 11 (4), 577-584.

Levy, F., Hay, D. A., McStephen, M., Wood, C., \& Waldman, I. (1997). Attention-Deficit Hyperactivity Disorder: A category or continuum? Genetic analysis of a large-scale twin study. Journal of the American Academy of Child and Adolescent Psychiatry, 36, 737-744.

Lovejoy, D. W., Ball, J. D., Keats, M., Stutts, M. L., Spain, E. H., Janda, L., et al. (1999). Neuropsychological performance of adults with attention deficit hyperactivity disorder (ADHD): Diagnostic classification estimates for measures of frontal lobe / executive functioning. Journal of the International Neuropsychological Society, 5 , 222-233.

Luteijn, F., \& van der Ploeg, F. A. (1983). Handleiding Groninger Intelligentietest (GIT) [Manual Groningen Intelligence Test]. Lisse, The Netherlands: Swets \& Zeitlinger. 
Manuzza, S., Klein, R. G., \& Moulton, J. L. (2003). Persistence of Attention-Deficit/ Hyperactivity Disorder into adulthood: What have we learned from the prospective follow-up studies? Journal of Attention Disorders, 7(2), 93-100.

Millstein, R. B., Wilens, T. E., Biederman, J., \& Spencer, T. J. (1997). Presenting ADHD symptoms and subtypes in clinically referred adults with ADHD. Journal of Attention Disorders, 2(3), 159-166.

Murphy, K., Barkley, R. A., \& Bush, T. (2001). Executive functioning and olfactory identification in young adults with Attention Deficit-Hyperactivity Disorder. Neuropsychology, 15, 211-220.

Murphy, K. R., Barkley, R. A., \& Bush, T. (2002). Young adults with Attention Deficit Hyperactivity Disorder: Subtype differences in comorbidity, educational, and clinical history. The Journal of Nervous and Mental Disease, 190(3), 147-157.

Nadeau, K. G. (1995). A comprehensive guide to attention deficit disorder in adults: Research, diagnosis, and treatment. New York: Brunner/Mazel.

Nigg, J. T., Butler, K. M., Huang-Pollock, C. L., \& Henderson, J. M. (2002). Inhibitory processes in adults with persistent onset ADHD. Journal of Co-sulting and Clinical Psychology, $70(1), 153-157$.

Nigg, J. T., Stavro, G., Ettenhofer, M., Hambrick, D. Z., Miller, T., \& Henderson, J. M. (2005). Executive functions and ADHD in adults: Evidence for selective effects on ADHD symptom domains. Journal of Abnormal Psychology, 114 (3), 706-717.

Quinlan, D. M., \& Brown, T. E. (2003). Assessment of short-term verbal memory impairments in adolescents and adults with ADHD. Journal of Attention Disorders, 6(4), 143-152.

Repovs, G., \& Baddeley, A. (2006). The multi-component model of working memory: Explorations in experimental cognitive psychology. Neuroscience, 139, 5-21.

Rey, A. (1964). L'examen psychologique dans les cas d'encephalopathie traumatique [psychological assessment in cases of traumatic brain injury]. Paris: Presses Universitaires de France.

Roth, R. M., Wishart, H. A., Flashman, L. A., Riordan, H. J., Huey, L., \& Saykin, A. J. (2004). Contribution of organizational strategy to verbal learning and memory in adults with Attention-Deficit/Hyperactivity Disorder. Neuropsychology, 18(1), 78-84.

Schmidt, M. (1996). Rey Auditory Verbal Learning Test: A Handbook. Los Angeles, California: Western Psychological Services.

Seidman, L. J., Biederman, J., Weber, W., Hatch, M., \& Faraone, S. V. (1998). Neuropsychological functioning in adults with attention-deficit hyperactivity disorder. Biological Psychiatry, 44, 260-268.

Sergeant, J. A., Geurts, H., \& Oosterlaan, J. (2002). How specific is a deficit of executive functioning for Attention-Deficit/Hyperactivity Disorder? Behavioral Brain Research, 130, 3-28.

Sternberg, R. J., \& Tulving, E. (1977). The measurement of subjective organization in free recall. Psychological Bulletin, 84(3), 539-556.

Stone, M., Gabrieli, J. D. E., Stebbins, G. T., \& Sullivan, E. V. (1998). Working and strategic memory deficits in Schizophrenia. Neuropsychology, 12 (2), 278-288.

Stuss, D. T., Alexander, m. P., Palumbo, C. L., Buckle, L., Sayer, L., \& Pogue, J. (1994). Organizational strategies of patients with unilateral or bilateral frontal lobe injury in word listing tasks. Neuropsychology, 8(3), 355-373. 
Van Boxtel, M. P. J., Buntinx, F., Houx, P. J., Metsemakers, J. F. M., Knottnerus, A., \& Jolles, J. (1998). The relation between morbidity and cognitive performance in a normal aging population. Journal of Gerontology, 53A (2), M147-M154.

Van der Elst, W., Van Boxtel, M. P. J., Van Breukelen, G. J. P., \& Jolles, J. (2005). Rey's verbal learning test: Normative data for 1855 healthy participants aged 24-81 years and the influence of age, sex, and mode of presentation. Journal of the International Neuropsychological Society, 11, 290-302.

Vanderploeg, R. D., Schinka, J. A., \& Retzlaff, P. (1994). Relationships between measures of auditory verbal learning and executive functioning. Journal of Clinical and Experimental Neuropsychology, 16(2), 243-252.

Verkes, R. J., Gijsman, H. J., Pieters, M. S. M., Schoemaker, R. C., de Visser, S., Kuijpers, M., et al. (2001). Cognitive performance and serotenergic function in users of ecstasy. Psychofarmacology., 153, 196-202.

Walker, A. J., Shores, E. A., Trollor, J. N., Lee, T., \& Sachdev, P. S. (2000). Neuropsychological functioning of adults with attention deficit hyperactivity disorder. Journal of Clinical and Experimental Neuropsychology, 22, 115-124.

Weiss, M., \& Murray, C. (2003). Assessment and management of attention-deficit hyperactivity disorder in adults. Canadian Medical Association Journal, 168(6), 715-722.

Woods, S. P., Lovejoy, D. W., \& Ball, J. D. (2002). Neuropsychological characteristics of adults with ADHD: A comprehensive review of initial studies. The Clinical Neuropsychologist, 16(1), 12-34.

Woods, S. P., Rippeth, J. D., Conover, E., Gongratana, A., Gonzalez, R., Carey, C. L., et al. (2005). Deficient strategic control of verbal encoding and retrieval in individuals with methamphetamine dependence. Neuropsychology, 19 (1), 35-43. 


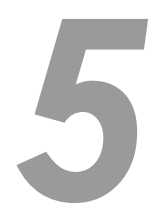

\section{Automatic and controlled processes underlying verbal fluency in adults with ADHD: Productivity over time, clustering and switching}




\section{Abstract}

This study examined automatic and controlled strategies on semantic category fluency (SCF) and initial letter fluency (ILF) in adult ADHD. Word production for each 15-s time slice, and word clustering, switching and errors over $60 \mathrm{~s}$ were calculated for both tasks. Sixteen adults with ADHD, combined type, were compared to fifteen age- and education-matched healthy controls. Thirteen ADHD subjects were taking methylphenidate (MPH) medication; the remainder three subjects were treatment naïve. Results showed that the ADHD adults generated a significantly lower number of words during the first $15 \mathrm{~s}$ as compared to healthy controls on the SCF. This was not found on the ILF. No group differences in controlled information processing, errors, clustering and switching strategies emerged on both types of fluency. Our results indicate that adults with ADHD have difficulty with automatic information processing from the semantic memory store, despite MPH treatment. These findings appear in ADHD patients on MPH medication and more likely reflect a pervasive ADHD-related deficit. 


\section{Introduction}

Verbal fluency tasks are widely used in clinical neuropsychological assessment and research to evaluate cognitive performance in adults with ADHD (Hervey, Epstein, \& Curry, 2004). The two most common types of fluency tasks are phonemic or initial letter fluency and semantic category fluency. On initial letter fluency (ILF), respondents have to generate as many words as possible beginning with a specified letter typically within $60 \mathrm{~s}$, while on semantic category fluency (SCF), they have to name exemplars that belong to a specified semantic category (e.g., animals) (Abwender, Swan, Bowerman, \& Connolly, 2001; Lezak, 1995; Monsch et al., 1994). Successful performance on these word fluency tests is dependent upon the integrity of memory (i.e., lexical and semantic search store), verbal attention, working memory, and executive processes (i.e., initiation, strategic retrieval) (Monsch et al., 1994).These are aspects of executive functioning that are impaired in ADHD (Barkley, 1998; Castellanos \& Tannock, 2002; J.T. Nigg, Butler, Huang-Pollock, \& Henderson, 2002).

A variety of studies indicate that, in comparison to healthy control subjects, adult patients with ADHD show disturbances on initial letter fluency (e.g., Boonstra, Oosterlaan, Sergeant, \& Buitelaar, 2005; Hervey et al., 2004; Woods, Lovejoy, \& Ball, 2002), while only two other studies found no differences between ADHD adults and healthy controls on this type of fluency (Barkley, Murphy, \& Kwasnik, 1996; Johnson et al., 2001). The ADHD adults produced generally fewer words compared to healthy controls. In addition, the only two studies examining semantic category fluency revealed that ADHD adults name fewer words than healthy subjects (Tucha et al.2005; Walker, Shores, Trollor, Lee, \& Sachdev, 2000). Initial letter fluency has been primarily regarded as a prominent and reliable measure to differentiate adults with ADHD from healthy controls (Woods et al., 2002). The reason for this is that this type of task likely requires more demanding processing and executive functions compared to semantic category fluency (Abwender et al., 2001; Lezak, 1995; Monsch et al., 1994).

A potential drawback among these prior studies in adult ADHD and verbal fluency is that they investigated performance on the most common fluency measure (irrespective of type of fluency) derived by the total number of correct words generated over $60 \mathrm{~s}$. Little is known about the underlying dynamic processes and strategies that these adults use to search through their memory store (semantic memory and episodic memory) when performing these tasks while using this outcome measure. The present paper presents the findings of the first study to examine the underlying automatic and controlled processes and strategies in ADHD adults. This broadening of scope has been achieved in two important ways.

First of all, recent experiments in children with ADHD (Hurks et al., 2004) and healthy adults (A.K. Troyer, 2000) have indicated that the pattern of words produced over time (defined in quartiles of $15 \mathrm{~s}$ ) provides more insight into automatic 
and controlled cognitive processing. It appears that in the first time quartile a ready pool of frequent words is available for production and is more automatically activated. As time passes this pool is exhausted and production becomes more effortful and less productive (Crowe, 1998). Successful performance on verbal fluency tasks therefore seems to depend both upon effectiveness of automatic and controlled information processing. Automatic processing is generally fast and relatively unconscious, while controlled processing is slow, effortful and more attention-demanding (Shiffrin \& Schneider, 1977). More specifically, Hurks et al. (2004) found that, while comparing ADHD children with healthy children, overall word productivity decreased markedly as a function of time in both groups, with the highest production in the initial time slice (0-15 s) on both fluency tasks. In addition, ADHD children had more problems generating words during the first time slice (15 s) of the initial letter fluency than healthy controls. No such differences were found with respect to the semantic category fluency (Hurks et al., 2004). These results suggested that children with ADHD had impaired automation of skills specific for initial letter fluency. They explained this by a maturation lag in learning of letters compared with more known and automatic access to semantic categories in children with ADHD. In addition, several studies have shown that children with ADHD display deficient automatic and/or controlled information processing strategies (Bocherding et al., 1988; Kalff et al., 2003). However, these findings still remain inconsistent. In adults with ADHD these automatic and controlled processes underlying verbal fluency performance are not yet well understood. The present study was devised to obtain more information on this subject. Given the cognitive development through adolescence and young adulthood we expected this automation problem on ILF to be less pervasive in the ADHD adults as learning of letters is presumed to be automated in young adulthood. We thereby expected more problems in their controlled information processing (e.g., the following time slices after the first $15 \mathrm{~s}$ ) given their persistent dysfunctions in more complex executive functions that demand more attentional resources (Hervey et al., 2004).

A second way to investigate low and high controlled processing in ADHD adults can be reached by studying the more rule-governed search strategies (Monsch et al., 1994) in the verbal fluency paradigm. Two main strategic component processes can be measured by use of verbal fluency tests, namely clustering (i.e., the production of words within semantic or letter subcategories) and switching (i.e., the ability to shift efficiently to a new subcategory that is not necessarily a new cluster) (A.K. Troyer, 2000; A. K. Troyer, Moscovitch, \& Winocur, 1997). Clustering has been regarded to depend on verbal memory and word storage relating primarily to temporal-lobe functioning (A.K. Troyer, 2000; A. K. Troyer et al., 1997; A. K. Troyer, Moscovitch, Winocur, Alexander, \& Stuss, 1998). Moreover, it has been argued that semantic clustering is more automatic relying on common rules of categorization (e.g., animals, pets) whereas letter clustering is more demanding and effortful (Abwender et al., 2001). Switching on the other 
hand involves, next to strategic search processes, cognitive flexibility and is a relatively effortful process. It has been primarily related to frontal-lobe functioning (A.K. Troyer, 2000; A. K. Troyer et al., 1997; A. K. Troyer et al., 1998). These latter authors further noted that clustering and switching abilities are equally related to performance on semantic category fluency. Initial letter fluency on the other hand relies more closely on switching, executive strategies alone, since there are few semantic clues in available. Given the dysfunctions in executive, organization skills and verbal memory which are mediated by frontal-lobe dysfunctioning in ADHD (Barkley, 1998; Barkley, Grodzinsky, \& DuPaul, 1992; Durston, 2003), carefully observing both of these cognitive processes would thereby be salient for a better understanding of the mechanisms involved in ADHD. Also, inefficient use of these strategies would possibly be a marker of executive dysfunction in ADHD. Little work has been done to date on executive strategies used for retrieval from the lexicon and semantic memory system in adults with ADHD (Hervey et al., 2004; Marchetta et al., submitted; Woods et al., 2002). Only one study investigating clustering and switching strategies on both types of fluency in adult ADHD showed that unmedicated adults with ADHD produced fewer switching responses on both fluency tasks and tended to generate smaller clusters on semantic category fluency relative to healthy controls (Tucha et al., 2005). In the present paper, we investigated these strategies in medicated ADHD adults and could therefore examine whether these reported deficits ameliorate when taking stimulant medication. Recent studies have reported that methylphenidate (MPH) affects cognitive functions, including verbal fluency total scores, in ADHD adults. Moreover, performance of the ADHD adults on MPH generally reaches a similar level of the healthy controls on such cognitive tasks (Kuperman et al., 2001; Riordan et al., 1999; Wilens, Spencer, \& Biederman, 2002).

Taken together, in the present study we investigated the performance of adults with ADHD and healthy control subjects on initial letter fluency and semantic category fluency. More specifically, the underlying automatic and controlled mechanisms including word productivity as a function of time, clustering and switching were of primary interest. We chose to focus on adults with the ADHD combined subtype (ADHD-C) as this is the most prevalent disorder in adult ADHD (Barkley, 1998; Manuzza, Klein, \& Moulton, 2003; K. R. Murphy, Barkley, \& Bush, 2002; Weiss \& Trokenberg-Hechtman, 1993). Also, in order to make generalizations possible as most studies are performed on this subtype. Furthermore, the majority of the ADHD adults in our study were taking stimulant medication methylphenidate at the time of testing and the remainder were treatment-naïve ADHD individuals. Thus, in contrast to previous studies, we examined whether these underlying mechanisms in verbal fluency remain impaired when patients with ADHD are on stimulant treatment. We hypothesized that adults with ADHD on stimulant medication would perform similar on automatic (first time slice of 15 s) and controlled information processing (next 16-60 s slices) specifically regarding initial letter fluency as compared to healthy controls. We also expected that 
performance on strategic retrieval processes, notably switching strategy, would be similar in ADHD adults and healthy controls.

\section{Method}

\section{Participants}

In the present study, the initial sample consisted of two groups ( $N=35)$ : 17 adults with ADHD (mean age $=28.8, S D=6.24$; range 21.9-41.9) and 18 edu-cation and age-matched normal control adults (mean age $=28.5, S D=6.44$; range 21.3 41.3). The ADHD group consisted of 16 individuals with combined attention and hyperactivity problems (ADHD-Combined) and one adult with mainly attention problems (ADHD- Inattentive). All participants were males and right-handed. Participants in the ADHD group were diagnosed with ADHD combined type or ADHD, predominantly inattentive type by an outside clinical professional. Also, they had a minimum score of five symptoms of inattention and/or hyperactivityimpulsivity on the Current Symptoms Scale and a score of at least four symptoms of inattention and/or hyperactivity-impulsivity on the retrospective Childhood Symptoms Scale (Barkley \& Murphy, 1998). Exclusion criteria for all participants were presence of any current Axis-I psychiatric diagnosis other than ADHD, IQ < 80, neurological trauma or disorder. An additional exclusion criterion for the control group was five or more symptoms of inattention and/ or hyperactivity on the Current Symptoms Scale and/ or Childhood Symptoms Scales. Participants of the ADHD group were recruited by spreading information brochures during group assessments of adults with ADHD and by advertisements on websites of ADHD associations. Control participants were recruited by advertisements in local newspapers in the southern parts of the Netherlands. All participants provided written informed consent for participation and received 50 euros after completion of the experiment. The study reported in the present paper was part of a larger crosssectional study on cognitive functions in adults with ADHD in which multiple researchers participated.

Table 1 summarizes the demographic and clinical data for the ADHD-combined type patients and healthy controls included in the present study. A total of four participants were excluded. One participant with ADHD (ADHD-Inattentive) displayed not enough symptoms of ADHD on both the ADHD Rating Scales (maximum of three symptoms per scale). Three participants of the control group displayed too many ADHD symptoms (five or more symptoms on the current and/or childhood ADHD scale). Therefore, the final sample consisted of 31 participants ( $n$ ADHD $=16$; $n$ healthy controls $=15$ ). In total, 13 ADHD participants were taking stimulant medication and 3 participants were treatment-naïve at the time of testing. The groups did not significantly differ with regard to age, educational level, and the performance of the Block Design subtest of the Wechsler Adult Intelligence Scale, as an estimate of general abilities (Wechsler, 1981). As 
expected, the ADHD patients displayed significantly more current and childhood ADHD symptoms than the controls and differed from the controls in their use of $\mathrm{MPH}$ medication.

Table 1. Demographic data for the ADHD and control groups

\begin{tabular}{|c|c|c|c|c|c|c|}
\hline & \multicolumn{2}{|c|}{$\begin{array}{l}\text { ADHD } \\
(n=16)\end{array}$} & \multicolumn{2}{|c|}{$\begin{array}{l}\text { Control } \\
(n=15)\end{array}$} & \multirow[b]{2}{*}{ ta } & \multirow[b]{2}{*}{$p$} \\
\hline & Mean & $S D$ & Mean & $S D$ & & \\
\hline Age & 29.1 & 6.3 & 29.4 & 6.6 & -.15 & .875 \\
\hline Education level b & 4.9 & 1.6 & 5.4 & 1.4 & -.82 & .416 \\
\hline Block Design T score & 65.3 & 7.0 & 65.6 & 8.4 & -.12 & .899 \\
\hline Medication (yes/no) c & $13 / 3$ & & $0 / 15$ & & 18.0 & $<.001$ \\
\hline No. current ADHD symptoms & 13.0 & 2.1 & 0.3 & .6 & 20.33 & $<.001$ \\
\hline No. childhood ADHD symptoms & 12.7 & 3.8 & 1.0 & 1.6 & 10.04 & $<.001$ \\
\hline No. inattention symptoms d & 1.2 & 1.4 & & & & \\
\hline $\begin{array}{l}\text { No. hyperactivity-impulsivity } \\
\text { symptoms }{ }^{d}\end{array}$ & 1.9 & 2.1 & & & & \\
\hline \multicolumn{7}{|c|}{ 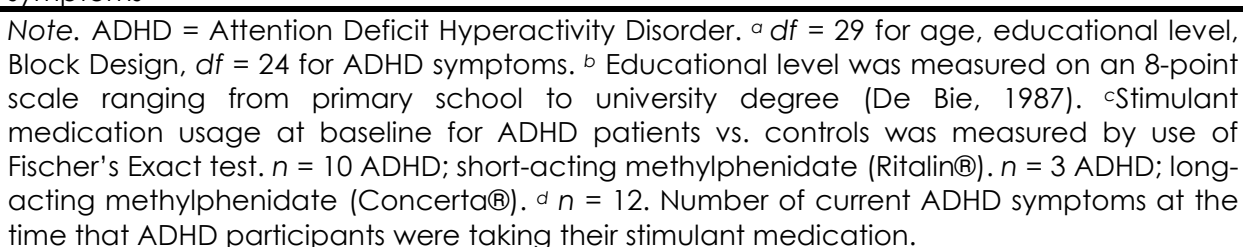 } \\
\hline
\end{tabular}

\section{Procedure}

All testing was conducted in Dutch and each participant was tested individually in a special testing room. Testing lasted two hours. The order of presentation of the tasks was the same for all participants, starting with the ILF task. These fluency tasks were part of a larger neuropsychological battery including the subtest Block Design of the WAIS-R and verbal memory tests. The present study focused only on the results of the verbal fluency tasks. After completion of the entire experiment all participants were asked to fill in the Current Symptoms Scale and Childhood Symptoms Scale.

\section{Measures}

ADHD Rating Scale for Adults (Barkley \& Murphy, 1998). This scale is a short, self-report screening questionnaire to assess ADHD in adults (Barkley \& Murphy, 1998; P. Murphy \& Schachar, 2000). Participants completed two versions of this scale, the Current Symptoms Scale, for current symptoms (past six months), and the Childhood years (Barkley \& Murphy, 1998). Both versions contain the 18 items, including nine items measuring inattention symptoms and nine items measuring hyperactivity/impulsivity symptoms, from the diagnostic criteria for ADHD in the Diagnostic and Statistical Manual of Mental Disorders (American Psychiatric Association [APA], 1994) with a slight adaptation in word use for the adult version as a 
Symptoms Scale, for recall of childhood symptoms between the ages 5 and 12 number of behaviors are not appropriate anymore (e.g., climbing). Each item is rated on a Likert scale from 0 to 3 , with $0=$ not at all or rarely, $1=$ sometimes, $2=$ often, and 3 = very often. A score is considered a symptom if an item is answered with 'often' or 'very often', leading to a maximum of 9 symptoms for inattention and 9 symptoms for hyperactivity/impulsivity (max. total ADHD score $=18$ ).

For participants with ADHD two copies of current symptoms were provided; one with symptoms of ADHD when they were taking MPH medication and one copy referring to symptoms during the treatment-naive period. Both copies consisted of the same items. ADHD participants who did not use stimulant medication only had to fill in the copy referring to the treatment-naïve period. For the present study, the current mean number of ADHD symptoms while on and off $\mathrm{MPH}$ medication and their mean childhood ADHD symptoms are presented in Table 1.

The WAIS-R subtest Block Design. The subtest Block Design of the WAIS-R measures visuospatial abilities and is frequently used as an estimation of general intelligence (Wechsler, 1981; Stinissen, Willems, Coetsier, \& Hulsman, 1970). Furthermore, if matched for level of education, no difference in per-formance between adults with ADHD and normal control is expected (Bridgett \& Walker, 2006; Seidman, Biederman, Weber, Hatch, \& Faraone, 1998). Therefore, in the present study, where groups are matched for level of education and for age, the Block Design subtest can be used to check for similar levels of general intelligence in both groups. The block design raw scores were transformed to standard T-scores $(M=50 ; S D=10)$ to compare these data.

The Verbal Fluency test (Hurks et al., 2004; Korkman, Kirk, \& Kemp, 1998). This subtest is part of the NEPSY (NEuroPSYchological investigation for children) - A Developmental Neuropsychological Assessment battery (NEPSY; Korkman et al., 1998) and is believed to measure retrieval from the memory store and cognitive information processing. It includes two types of verbal fluency, namely semantic category fluency (SCF) and initial letter fluency (ILF). Semantic Category Fluency subtask (SCF): In this subtask participants were required to name as many different animals as possible in $60 \mathrm{~s}$. Initial Letter Fluency sub task (ILF): In this subtask participants were asked to generate as many words beginning with the letter $M$ as possible and $60 \mathrm{~s}$ were allowed, excluding proper human names and cities (according to standard instructions). According to the Van Loon list presenting image-ability ratings of Dutch words (Van Loon, 1985), words that begin with the letters $M$ are relatively common in the Dutch language $14.3 \%$ of the Dutch words begin with this letter). A similar procedure using the same letter and semantic category of the described verbal fluency test has been applied in healthy adults in a Dutch population (Van der Elst, Van Boxtel, Van Breukelen, \& Jolles, 2006). 
The following scores were analyzed for both fluency subtests: (1) total number of correct words generated over (a) 1-15 s, (b) 16-30 s, (c) 31-45 s, and (d) 46$60 \mathrm{~s}$, (2) total number of intrusive errors (i.e., words not beginning with the appropriate letter or words not belonging to the predefined category) over $60 \mathrm{~s}$, (3) total number of perseverations (i.e., repetitions of correct words, morphological variants) over $60 \mathrm{~s}$. Automatic processing was defined as performance on the first $15 \mathrm{~s}$ of either type of fluency. Controlled processing was defined as performance over the succeeding $45 \mathrm{~s}$ of each test. The number of clusters, mean cluster size and switches were calculated according to the scoring methods developed by Troyer et al. $(1997,2000)$. Clusters consisted of two or more successively generated words belonging to the same semantic or letter subcategory, including perseverations and intrusions as a measure of retrieval from verbal memory. In contrast to the scoring rules by Troyer et al. (2000), single words were not counted as clusters, because they suggest a failure to retrieve other words from that particular category (Koren, Kofman, \& Berger, 2005). A phonemic cluster included two or more successive words beginning with at least the same first two letter sounds (e.g., month, mother), differing only by one vowel sound (e.g., miss, mess), differing by the last consonant sound (e.g., mess, met) or rhymes. A semantic cluster was defined as two generated words belonging to the same semantic subcategory, including zoological categories, such as fish, feline, canine, insects, etc. Mean cluster size was calculated by dividing the total number of words in clusters by the number of clusters produced for each fluency subtest (size $=$ number of words in a cluster minus 1 with a cluster of two words = mean cluster size of 1), excluding single words with cluster size of 0 as a measure of retrieval. Switches were calculated as the total number of transitions between clusters, also including switching between single words (e.g., a phonemic cluster: 'miss mess' followed by 'magic') as a measure of cognitive flexibility. The raw number of switches was utilized as outcome measure as suggested by Troyer et al. (1998).

\section{Statistical analysis}

All data were first screened for violations of the assumptions of the parametric tests (independent $t$ - tests, GLM repeated measures). Nonparametric statistics (Mann-Whitney signed ranked tests) were used for not normally distributed data. As these were comparable to the $t$ - tests, parametrics are reported here. The rejection criterion was set at $p<.05$, two-tailed. General Linear Model (GLM) repeated measures analysis was used to examine word productivity over time with Time interval $(0-15,16-30,31-45,46-60)$ as dependent variable, Type of fluency (SCF vs. ILF) as within-subject factor and group (ADHD versus control) as between-subject factor. Bonferroni corrections were made for post-hoc multiple comparisons.

The raw intrusion and perseveration error scores were categorized into three levels (i.e., zero errors, one error, and more than one error) based on their 
distribution. For each type of errors, the raw data were collapsed over the time intervals for each fluency task. The data of each type of errors were separately compared for group performance by use of the Chi square tests to evaluate group differences (ADHD and control) on types of error (categorized into three levels) for each fluency task with Monte-Carlo corrections for empty cells.

\section{Results}

Word production over time and type of fluency

One ADHD participant was excluded from the word productivity over time analyses, because of extreme value ( $>3$ times interquartile standard deviation from the median). A 2 (Type of fluency) $\times 4$ (Time interval) $\times 2$ (Group) repeated measures design revealed significant main effects for Type of fluency task (Type: $\left.F(3,81)=89.50, p<.001, n p^{2}=.76\right)$, word production as a function of time (Time interval: $\left.F(3,81)=83.80, p<.001, n p^{2}=.75\right)$, and an interaction between these factors (Type $\times$ Time interval: $F(3,81)=11.70, p<.001, n p^{2}=.30$ ). Overall, the participants generated significantly more words on the SCF task (mean $=22.9$ [SD = 5.6]) than on the ILF (mean = 12.47 [SD = 4.4]) (see also Table 2). Secondly, word productivity decreased significantly with each succeeding time slice of $15 \mathrm{~s}$, averaged over both types of fluen-cy and groups (mean1-15s $=7.2$, mean16-30s $=$ 4.3, mean31-45s $=3.4$, mean $46-60 s=2.4$ ). Thirdly, the significant Type $\times$ Time interval interaction indicated that the decrease in word production was not parallel for SCF and ILF, with a steeper decrease in the SCF, although in both tasks word production decreased with time. There was no significant between-subject effect for group, $F(1,27)=.90, p=.351, n p^{2}=.03$ (see also Table 2 separated per task). Furthermore, there was no interaction between group and words generated over the different time slices (Group $\times$ Time interval: $F(3,81)=2.33, p=.500, n p^{2}=$ .02). In contrast, a significant Group by Type of fluency interaction (Group $\times$ Type: $\left.F(1,81)=6.42, p=.017, n p^{2}=.19\right)$ was found, suggesting that the ADHD adults produced more words on the ILF than controls whereas the controls produced more words on the SCF. Lastly and most important, there was an interaction of Group by Time by Type of fluency, $F(3,81)=2.82, p=.044, n p^{2}=.09$ ) that indicated that performance of the groups was not linear across the different time intervals and types of fluency. Figure 1 depicts group performance over time intervals for each type of fluency (SCF and ILF) where the ADHD adults seem to perform better on first time slice of ILF compared to controls and vice versa for the healthy controls on SCF.

Additional GLM multivariate testing revealed a significant main effect of Group on the first $15 \mathrm{~s}$ time slice of the SCF task, $F(1,28)=4.95, p=.034, n p^{2}=.15$; the ADHD adults (mean $=8.4$ ) had a significantly lower performance than the healthy controls (mean $=10.4$ ) on this first time interval. No other significant main 
effects of group were found on the other time slices for SCF (all $p>$.10). No main effects of Group were found on the four time intervals of the ILF task (all ps > .10).

Table 2. Fluency data for the ADHD and control groups with and without medication

\begin{tabular}{|c|c|c|c|c|c|c|}
\hline \multirow[b]{2}{*}{ Variable } & \multicolumn{2}{|c|}{ ADHD } & \multicolumn{2}{|c|}{ Control } & \multirow[b]{2}{*}{$t / x^{2}$} & \multirow[b]{2}{*}{$p$} \\
\hline & Mean & $S D$ & Mean & $S D$ & & \\
\hline \multicolumn{7}{|l|}{ SCF } \\
\hline Correct words generated & 21.0 & 4.2 & 24.6 & 5.9 & -1.90 & .068 \\
\hline Switches & 14.3 & 3.7 & 14.3 & 4.5 & .02 & .978 \\
\hline Clusters & 4.8 & 1.6 & 5.2 & 1.8 & -.61 & .543 \\
\hline Mean cluster size & 1.5 & .5 & 1.9 & 1.1 & -1.11 & .274 \\
\hline Intrusions: $0 / 1 / \geq 2$ & $10 / 4 / 2$ & & $12 / 3 / 0$ & & 1.97 & .519 \\
\hline Perseverations: $0 / 1 / \geq 2$ & $11 / 5 / 0$ & & $13 / 2 / 0$ & & 1.42 & .233 \\
\hline \multicolumn{7}{|l|}{ ILF } \\
\hline Correct words generated & 13.0 & 3.4 & 11.5 & 5.1 & .91 & .372 \\
\hline Switches & 10.3 & 3.0 & 9.0 & 3.6 & 1.03 & .311 \\
\hline Clusters & 2.0 & 1.1 & 1.4 & 1.2 & 1.22 & .230 \\
\hline Mean cluster size & 1.2 & .6 & .9 & .7 & 1.05 & .299 \\
\hline Intrusions: $0 / 1 / \geq 2$ & $13 / 2 / 0$ & & $12 / 3 / 0$ & & .24 & .624 \\
\hline Perseverations: $0 / 1 / \geq 2$ & $10 / 5 / 0$ & & $12 / 2 / 1$ & & 2.33 & .394 \\
\hline
\end{tabular}

Note. ADHD = Attention Deficit Hyperactivity Disorder. SCF = Semantic Category Fluency. ILF = Initial Letter Fluency.
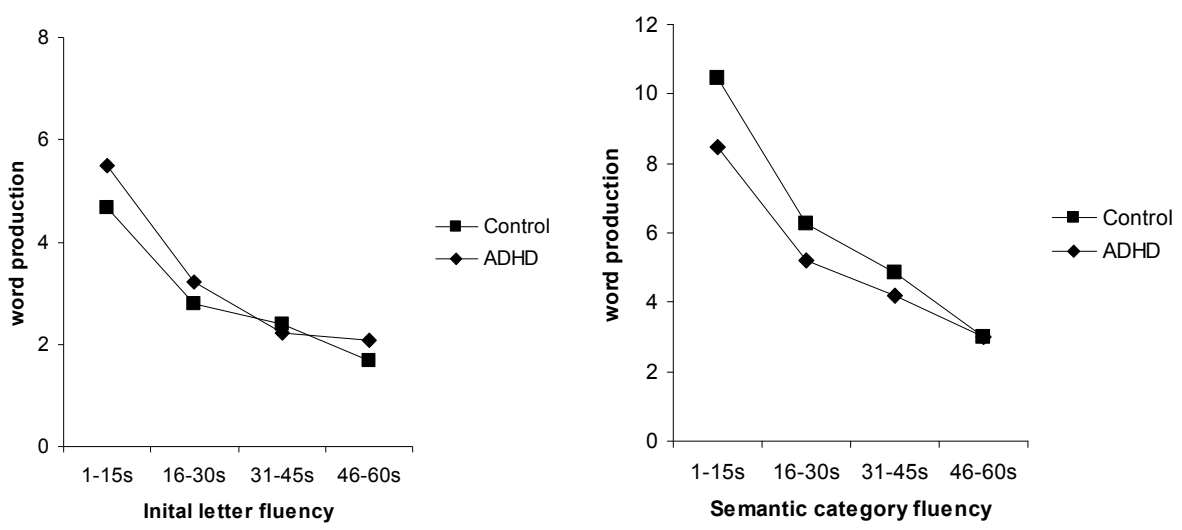

Figure 1. Word productivity scores over different time intervals for groups and type of fluency (semantic category fluency versus initial letter fluency) 
Clustering, switching and type of fluency

One ADHD participant was excluded from the analyses of total clusters, because of extreme value (> 3 times interquartile range from the median). A 2 (Type of fluency) $\times 2$ (Group) repeated measures design for total clusters re-vealed a significant main effect for Type of fluency (Type: $F(1,27)=105.13, p<.001, n p^{2}=$ .79) and a significant Group by Type of fluency interaction (Group $\times$ Type: $F(1$, $\left.27)=5.27, p=.030, n p^{2}=.16\right)$, but no main effect of group, $F(1,27)=.04, p<.838$, $n p^{2}=.00$. Overall, significantly more semantic clusters (mean $=4.8[S D=1.5]$ ) were produced on the SCF than phonemic clusters (mean $=1.76$ [SD = 1.2]) on ILF (see also Table 2). In addition, adults with ADHD seemed to produce more phonemic clusters than controls while controls produced more semantic clusters than ADHD adults as can be seen in Figure 2 left. However, additional post-hoc $t$-tests revealed no significant group differences on either fluency task on this measure as can be seen in Table 2. Furthermore, $t$ - tests revealed no group differences in mean cluster size over $60 \mathrm{~s}$ on semantic category or initial letter fluency (see Table 2).
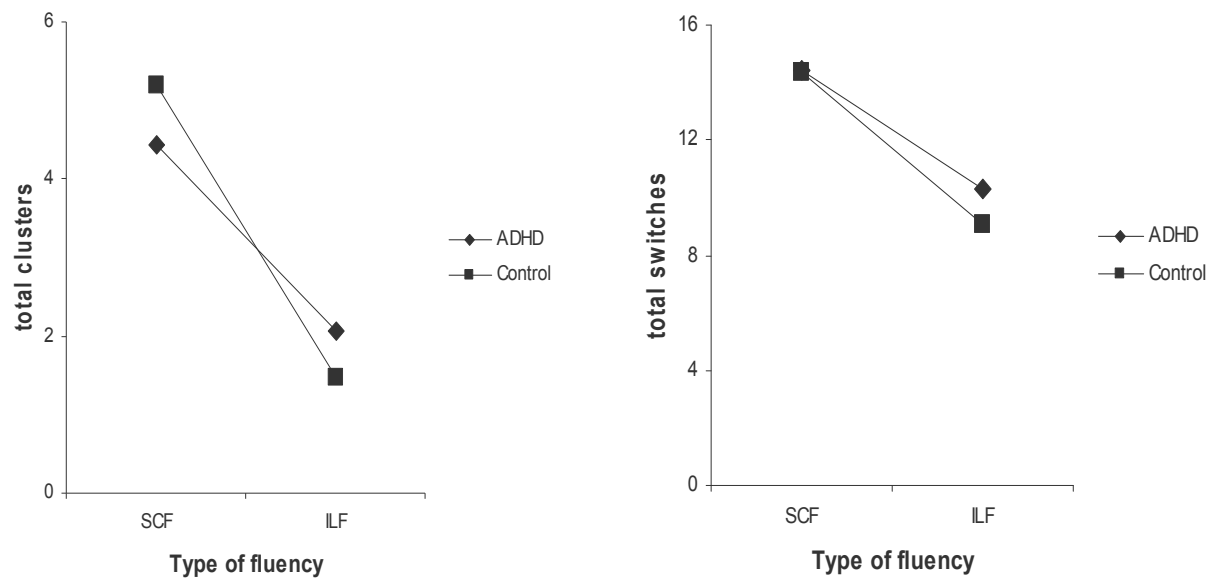

Figure 2. Total cluster scores for groups and type of fluency (semantic category fluency versus initial letter fluency; left). Total switch scores for groups and type of fluency (semantic category fluency versus initial letter fluency; right)

Finally, the 2 (Type of fluency) $\times 2$ (Group) repeated measures design for total switches revealed a significant main effect for Type of fluency $($ Type: $F(1,28)=$ $\left.25.69, p<.001, n p^{2}=.47\right)$, but no Group by Type of fluency interaction $(p=.520)$ or significant between-subject effect of Group $(p=.532)$ as depicted in Figure 2 right. Overall, the participants in both groups made more switches on the SCF $($ mean $=14.3[S D=4.1])$ than on the ILF (mean = 9.7 [SD = 3.3]). 


\section{Error analysis}

The chi square tests indicated no group differences in the number of intrusions and perseverations over total 60 s for both SCF and ILF (see Table 2 all ps > .20).

Analyses without medication-naive participants

All analyses described above are based on all ADHD participants, thereby including those three participants that were treatment-naive, to increase the power of the analyses. In addition, we performed all analyses excluding these three ADHD participants, revealing comparable results. Therefore, we found it justified to report on the analyses based on the total ADHD sample (on stimulant treatment and treatment-naïve). Furthermore, post-hoc multivariate testing for word productivity over time revealed a main effect of group on the first time interval of ILF. ADHD adults produced significantly more words in the first $15 \mathrm{~s}$ compared to healthy controls, indicating that ADHD adults with stimulant medication may have an even more improved performance on automatic processing on initial letter fluency.

\section{Discussion}

The aims of the present study were to investigate the automatic and controlled processes, and the search strategies underlying controlled performance on semantic category (SCF) and initial letter fluency (ILF) in adults with ADHD-Combined type. Our data revealed that the ADHD patients showed specific weak performance on automatic information processing on SCF, but not in their controlled information processing nor in their use of strategies in either fluency task relative to healthy controls. In addition, the ADHD adults with methylphenidate medication showed better performance in automatic processing on the ILF compared to controls.

With regard to the first aim, our findings provide support for unimpaired automatic and controlled processing on ILF in contrast to impaired automatic processing on SCF in adults with ADHD relative to controls. Moreover, our data showed a significant decrease in word productivity with time interval on semantic category fluency and initial letter fluency tasks for both groups. Overall, the adults generated more correct words over the first time slice $(0-15 \mathrm{~s})$ compared to the time slices that followed. This is also in line with findings from previous studies of children with ADHD (Hurks et al., 2004) and healthy adults (Crowe, 1998) indicating that this pattern can be extended to ADHD adults. Interestingly, we found by post-hoc multivariate analyses that the ADHD adults produced significantly fewer words during the first 15 s on semantic category fluency. No such 
group differences were found for word production over time on ILF. In contrast, our ADHD adults with MPH medication even generated more words on the first $15 \mathrm{~s}$ of ILF than the healthy controls. Our finding therefore suggests that the adults with ADHD have difficulty in their automating skills of processing semantic categorical information. This finding also remained consistent when excluding ADHD participants that were treatment-naïve. Thus, we suggest that this deficit in semantic automatic processing is more likely to be a pervasive ADHD-related deficit. These ADHD adults though seem to be better capable compensating for their difficulties in more controlled information processing on either SCF or ILF with stimulant treatment. These controlled processes are more effortful and demanding suggesting that methylphenidate is more likely to affect the more complex cognitive processes in ADHD adults (Kuperman et al., 2001; Riordan et al., 1999; Roth et al., 2004). It can be that these search strategies do not enable the ADHD adults enough to perform optimal in the beginning of a semantic category fluency test and other verbal memory strategies may be involved in their impaired automatic processing that were not investigated in the present study. For example, deficits in semantic clustering in word list learning have been found in ADHD adults relative to healthy controls (Hervey et al., 2004). This strategic deficiency has been attributed to a verbal working memory impairment, in particular the phonological loop that is involved in storage of verbal information (Hervey et al., 2004; Repovs \& Baddeley, 2006). Further research on this topic is necessary to find suitable explanations for this result.

The second aim of the present study, namely to investigate the search strategies underlying controlled performance in adults with ADHD. Our findings provided support in that patients with ADHD while taking MPH medication showed no impaired clustering and switching strategies com-pared to healthy control subjects. We found that all adults generated more clusters, but also more switches on the semantic category fluency compared to initial letter fluency. This result was also supported by our exploratory correlation data in that clustering and switching was moderate to high associated with semantic fluency performance over time. It was also in line with our expectation and is commonly reported in the literature (Abwender et al., 2001; Azuma, 2004; Crowe, 1998). Furthermore, our results showed that ADHD adults did not differ significantly from healthy controls concerning lower controlled clustering and high controlled switching strategies, regardless of fluency task. Based on a previous study by Tucha et al. (2005) that found switching impairments in adults with ADHD, we suggest that stimulant treatment may positively influence switching between clusters in adults with ADHD. In addition, effective switching requires shifting from subcategory to subcategory by disengaging from a previous strategy and initiate a new one that is more related to higher order executive functions and to be more related to ILF task (A. K. Troyer et al., 1997). In the present study, exploratory correlations also revealed that switching had the highest association with controlled processing on the ILF task. Based on these findings, we contend that stimulant medication in 
ADHD adults with the combined type plays a contributing role in different loads on controlled information processing strategies and executive functions, irrespective of type of fluency.

A limitation of the present study is the relatively small sample size, which may have reduced the statistical power. Replication in a larger adults ADHD sample also including other subtypes of ADHD would provide more insight into the potential different mechanisms involved in these ADHD subtypes and the influence of MPH treatment on these mechanisms. Recently, it has been suggested that the ADHD predominant inattentive adults are believed to have more problems with attention and organization (J. T. Nigg et al., 2005). Despite this, our findings add to the recent literature, in that we investigated not overall fluency performance, but more cognitive processes over time and also extended research on executive search strategies in medicated ADHD adults. Future research examining the efficacy of stimulant medication on verbal fluency and underlying mechanisms by use of more controlled clinical trials comparing ADHD adults on and off medication would extend our knowledge about neuropathology involved in ADHD.

The present results have several implications. Our findings indicated that the traditional scoring performance on both fluency tasks could not discriminate between the ADHD adults and controls. On the contrary, word productivity over time was a better marker for verbal fluency performance, in particular in detecting disturbances on a semantic category fluency task. Therefore, we suggest that this new variable should be included as an additional measure of verbal fluency and information processing in neuropsychological testing of adult ADHD. More normative research on the interval scores for healthy adults and ADHD adults is therefore warranted. For instance, developing norms and cut-off scores for the first time slice in comparison to the other three time slices would be an additional tool in the detection of an ADHD pattern during neuropsychological testing.

In conclusion, the ADHD adults primarily have difficulty in their automatic information processing of specific retrieval of semantic categorical information, but not in their controlled processing. In contrast, no group differences between ADHD patients and healthy controls in automatic and controlled information processing on a more difficult initial letter fluency task emerged. We suggest that word productivity over time is a more sensitive measure in detecting verbal fluency deficits in adults with ADHD than traditional total outcome measure. We contend that specific automatic information processing problems on SCF seems to be rather inherent to ADHD-Combined type symptomatology. Finally, our findings indicate that MPH stimulants may affect more strongly frontal-related automatic and controlled information processing in adults with ADHD. 
114 | Chapter 5

\section{Acknowledgement}

We thank Katya Bakker for her major role in test assistance and Wim van der Elst for his personal communication in preparing this manuscript. We also thank all those who kindly volunteered to participate in this study. 


\section{References}

Abwender, D. A., Swan, J. G., Bowerman, J. T., \& Connolly, S. W. (2001). Qualitative analysis of verbal fluency output: Review and comparison of several scoring methods. Assessment, 8(3), 323-336.

American Psychiatric Association (1994). Diagnostic and statistical manual of mental disorders (4th ed.). Washington DC: American Psychiatric Association.

Azuma, T. (2004). Working memory and perseveration in verbal fluency. Neur-psychology, $18(1), 69-77$.

Barkley, R. A. (1998). Attention-Deficit/ Hyperactivity Disorder: A handbook for diagnosis and treatment (2nd ed.). New York: The Guilford Press.

Barkley, R. A., Grodzinsky, G., \& DuPaul, G. J. (1992). Frontal lobe functions in attention deficit disorder with and without hyperactivity: a review and research report. Journal of Abnormal Child Psychology, 20, 163-188.

Barkley, R. A., Murphy, K., \& Kwasnik, D. (1996). Psychological adjustment and adaptive impairments in young adults with ADHD. Journal of Attention Disorders, 1 (1), 41-54.

Barkley, R. A., \& Murphy, K. R. (1998). Attention deficit hyperactivity disorder: A clinical workbook. New York: Guilford.

Bocherding, B., Thompson, K., Kruesi, M., Bartko, J., Rapoport, J. L., \& Weingartner, H. (1988). Automatic and effortful processing in attention deficit/hyperactivity disorder. Journal of Abnormal Child Psychology, 16, 333-345.

Boonstra, A. M., Oosterlaan, J., Sergeant, J. A., \& Buitelaar, J. K. (2005). Executive functioning in adult ADHD: a meta-analytic review. Psychological Medicine, 35, 1097-1 108.

Bridgett, D. J., \& Walker, M. E. (2006). Intellectual functioning in adults with ADHD: A metaanalytic examination of full scale IQ differences between adults with and without ADHD. Psychological Assessment, 18(1), 1-14.

Castellanos, F. X., \& Tannock, R. (2002). Neuroscience of attention-deficit/hyperactivity disorder: the search for endophenotypes. Nature Reviews Neuroscience, 3, 617-628.

Crowe, S. F. (1998). Decrease in performance on the verbal fluency test as a function of time: Evaluation in a young healthy sample. Journal of Clinical and Experimental Neuropsychology, 20(3), 391-401.

De Bie, S. E. (1987). Standaardvragen 1987: Voorstellen voor uniformering van vraagstellingen naar achtergrondkenmerken en interviews [Standard questions 1987: Proposal for uniformisation of questions regarding background variables and interviews] (2nd ed.). Leiden, the Netherlands: Leiden University Press.

Durston, S. (2003). A review of the biological bases of ADHD: What have we learned from imaging studies. Mental Retardation and Developmental Disabilities, 9, 184-195.

Hervey, A. S., Epstein, J. N., \& Curry, J. F. (2004). Neuropsychology of adults with AttentionDeficit/Hyperactivity Disorder: A meta-analytic review. Neuropsychology, 18(3), 485503.

Hurks, P. P. M., Hendriksen, J. G. M., Vles, J. S. H., Kalff, A. C., Feron, F. J. M., Kroes, M., et al. (2004). Verbal fluency over time as a measure of automatic and controlled processing in children with ADHD. Brain and Cognition, 55, 535-544.

Johnson, D. E., Epstein, J. N., Waid, L. R., Latham, P. K., Voronin, K. E., \& Anton, R. F. (2001). Neuropsychological performance deficits in adults with attention deficit/hyperactivity disorder. Archives of Clinical Neuropsychology, 16, 587-604. 
Kalff, A. C., De Sonnevile, L. M. J., Hurks, P. P. M., Steyaert, J., Zeben, T. M. C. B. V., Vles, J. S. H., et al. (2003). Low- and high-level controlled processing in executive motor control tasks in 5-6-year-old children at risk of ADHD. Journal of Child Psychology and Psychiatry, 44(7), 1049-1057.

Koren, R., Kofman, O., \& Berger, A. (2005). Analysis of word clustering in verbal fluency of school-aged children. Archives of Clinical Neuropsychology, 20, 1087-1104.

Korkman, M., Kirk, U., \& Kemp, S. L. (1998). NEPSY. A developmental neuropsychological assessment. San Antonio, TX: The Psychological Corporation.

Kuperman, D., Perry, P. J., Gaffney, G. R., Lund, B. C., Bever-Stille, K. A., Arndt, P. D., et al. (2001). Bupropion SR vs. methylphenidate vs. placebo for Attention Deficit Hyperactivity Disorder in adults. Annals of Clinical Psychiatry, 13(3), 129-134.

Lezak, M. D. (1995). Neuropsychological assessment (3rd ed.). Oxford: Oxford University Press.

Manuzza, S., Klein, R. G., \& Moulton, J. L. (2003). Persistence of Attention-Deficit/ Hyperactivity Disorder into adulthood: What have we learned from the prospective follow-up studies? Journal of Attention Disorders, 7(2), 93-100.

Marchetta, N. D. J, Hurks, P. P. M., Meijs, C. J. C., Scholtissen-In de Braek, D., Rozendaal, N., \& Jolles, J. (submitted). Verbal Learning and Organisational Strategies in Adults with ADHD: ADHD Subtypes and the Impact of Comorbidity.

Monsch, A. U., Bondi, M. W., Butters, N., Paulsen, J. S., Salmon, D. P., Brugger, P., et al. (1994). A comparison of category and letter fluency in Alzheimer's disease and Huntington's disease. Neuropsychology, 8(1), 25-30.

Murphy, K. R., Barkley, R. A., \& Bush, T. (2002). Young adults with Attention Deficit Hyperactivity Disorder: Subtype differences in comorbidity, educational, and clinical history. The Journal of Nervous and Mental Disease, 190(3), 147-157.

Murphy, P., \& Schachar, R. (2000). Use of self-ratings in the assessment of symptoms of Attention Deficit Hyperactivity Disorder in adults. American Journal of Psychiatry, 157, 1156-1159.

Nigg, J. T., Butler, K. M., Huang-Pollock, C. L., \& Henderson, J. M. (2002). Inhibitory processes in adults with persistent onset ADHD. Journal of Consulting and Clinical Psychology, 70(1), 153-157.

Nigg, J. T., Stavro, G., Ettenhofer, M., Hambrick, D. Z., Miller, T., \& Henderson, J. M. (2005). Executive functions and ADHD in adults: Evidence for selective effects on ADHD symptom domains. Journal of Abnormal Psychology, $114(3), 706-717$.

Repovs, G., \& Baddeley, A. (2006). The multi-component model of working memory: Explorations in experimental cognitive psychology. Neuroscience, 139, 5-21.

Riordan, H. J., Flashman, L. A., Saykin, A. J., Frutiger, S. A., Carroll, K. E., \& Huey, L. (1999). Neuropsychological correlates of methylphenidate treatment in adult ADHD with and without depression. Archives of Clinical Neuropsychology, 14(2), 217-233.

Roth, R. M., Wishart, H. A., Flashman, L. A., Riordan, H. J., Huey, L., \& Saykin, A. J. (2004). Contribution of organizational strategy to verbal learning and memory in adults with Attention-Deficit/Hyperactivity Disorder. Neuropsychology, 18(1), 78-84.

Seidman, L. J., Biederman, J., Weber, W., Hatch, M., \& Faraone, S. V. (1998). Neuropsychological functioning in adults with attention-deficit hyperactivity disorder. Biological Psychiatry, 44, 260-268. 
Shiffrin, R. M., \& Schneider, W. (1977). Controlled and automatic human information processing: II. Perceptual learning, automatic attending, and a general theory. Psychological Review, 84, 127-190.

Stinissen, J., Willems, P. J., Coetsier, P., \& Hulsman, W. L. L. (1970). Handleiding bij de Nederlandstalige bewerking van de Wechsler Adult Intelligence Scale (WAIS). Lisse, Netherlands: Swets \& Zeitlinger.

Troyer, A. K. (2000). Normative data for clustering and switching on verbal fluency tasks. Journal of Clinical and Experimental Neuropsychology, 22, 370-378.

Troyer, A. K., Moscovitch, M., \& Winocur, G. (1997). Clustering and switching as two components of verbal fluency: Evidence from younger and older healthy adults. Neuropsychology, $11(1), 138-146$.

Troyer, A. K., Moscovitch, M., Winocur, G., Alexander, M. P., \& Stuss, D. (1998). Clustering and switching on verbal fluency: The effects of focal frontal- and temporal-lobe lesions. Neuropsychologia, 36(6), 499-504.

Tucha, O., Mecklinger, L., Laufkötter, R., Kaunzinger, I., Paul, G. M., Klein, H. E., et al. (2005). Clustering and switching on verbal and figural fluency functions in adults with attention deficit hyperactivity disorder. Cognitive Neuropsychiatry, 10(3), 231-248.

Van der Elst, W., Van Boxtel, M. P. J., Van Breukelen, G. J. P., \& Jolles, J. (2006). Normative data for the animal, profession and letter $m$ naming verbal fluency tests for Dutch speaking participants and the effects of age, education, and sex. Journal of the International Neuropsychological Society, 12, 80-89.

Van Loon, A. (1985). Voorstelbaarheidswaarden van Nederlandse woorden. Lisse, the Netherlands: Swets and Zeitlinger.

Walker, A. J., Shores, E. A., Trollor, J. N., Lee, T., \& Sachdev, P. S. (2000). Neuropsychological functioning of adults with attention deficit hyperactivity disorder. Journal of Clinical and Experimental Neuropsychology, 22, 115-124.

Wechsler, D. (1981). Manual for the Wechsler Adult Intelligence Scale-Revised. New York: Psychological Corporation.

Weiss, G., \& Trokenberg-Hechtman, L. (1993). Hyperactive children grown up. New York: The Guilford Press.

Wilens, T. E., Spencer, T. J., \& Biederman, J. (2002). A review of pharmacotherapy of adults with attention-deficit/hyperactivity disorder. Journal of Attention Disorders, 5, 189202.

Woods, S. P., Lovejoy, D. W., \& Ball, J. D. (2002). Neuropsychological characteristics of adults with ADHD: A comprehensive review of initial studies. The Clinical Neuropsychologist, $16(1), 12-34$ 



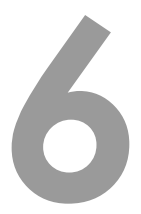

\section{Effect of stimulant medication on response inhibition and attention in adults with attention deficit hyperactivity disorder}




\begin{abstract}
The present study examined the efficacy of stimulant medication methylphenidate (MPH) on inhibition and attention in adult ADHD. This was done to investigate whether a primary disorder of inhibitory control or a more general deficit in inattention is apparent in adult ADHD. Thirteen adults diagnosed with ADHD combined type were compared to 15 healthy participants, matched for age and educational level, on a stop-signal task. The ADHD adults were assessed while on and off MPH medication and the healthy controls were also assessed on both these sessions. Results showed that ADHD adults off MPH medication had a slower stop-signal reaction time (SSRT), a larger variability in go response speed (SDRT), and made more choice errors than the healthy controls. Furthermore, choice error rate, but no other measures of inhibition and attention, was specifically improved in the ADHD adults when taking MPH medication. Our findings suggest that ADHD in adults is characterized by a more global deficit in attention regulation affecting inhibition.
\end{abstract}




\section{Introduction}

ADHD is characterised by two main symptom clusters of inattention and hyperactivity-impulsivity (American Psychiatric Association [APA], 2000). Since several years it is known that ADHD is not only confined to childhood, but also persists into adulthood in at least 30-60\% of the cases (Biederman et al., 1998; Manuzza \& Klein, 2000; Weiss \& Trokenberg-Hechtman, 1993). Compared to children with $A D H D$, little is known about cognitive functioning in adults with ADHD. During recent years, research is increasing on the study of executive functions in adults with ADHD (see review Hervey, Epstein, \& Curry, 2004; Lijfijt, Kenemans, Verbaten, \& van Engeland, 2005). Prior theories state that a deficient response inhibition is the core mechanism of ADHD (Barkley, 1997; Quay, 1997). In contrast, other theorists argue that ADHD is characterized by a more general deficit in inattention that could explain the deficient inhibitory control (Castellanos \& Tannock, 2002). This suggests that adults with ADHD have a deficit in response inhibition as well as an attention regulation problem. Methylphenidate (MPH) is worldwide the most prescribed stimulant drug in the treatment of adults with ADHD, yielding positive clinical effects on the ADHD symptoms in 60-70 \% of the patients (Wilens, Spencer, \& Biederman, 2002). Unfortunately, research on the effect of stimulant drugs (e.g., MPH) on these 'core symptoms' in adults with ADHD is still limited. This is an enormous lack as examining the efficacy of MPH would shed more light on which processes, inhibitory control or attention or both, are influenced by this stimulant medication. The aim of the present study is therefore to examine the underlying cognitive deficit(s) in adults with ADHD while on and off stimulant medication by use of a sensitive neurocognitive task, namely the Stop Signal Task which is a measure of complex reaction time.

The Stop Signal Task (SST) is often used as a measure of (1) the ability to stop unwanted prepotent responses and (2) attention regulation. It consists of two cognitive processes: the stopping process and the execution process (G. D. Logan, 1994; G. D. Logan, Schachar, R.J., \& Tannock, R., 1997; Schachar, Mota, Logan, Tannock, \& Klim, 2000). Stopping performance can be estimated as the speed of the stopping process, called the stop signal reaction time (SSRT) (G. D. Logan, 1994). Decrements in the stop process, for example by increases in the SSRT have been regarded as deficits in behavioral inhibition (G. D. Logan, 1994). In addition to inhibition, the SST measures several other variables of cognitive processing (G. D. Logan, 1994). More specifically, performance on the go task or execution process (expressed by mean RT [MRT], variability of RT [SDRT], omissions, and discrimination errors) provides insight into the ability to focus and maintain attention on the task. Go task performance can thus be regarded as an index of general attention, that may affect efficacy of inhibitory control (Castellanos \& Tannock, 2002). Therefore, the SST is commonly used to investigate both inhi-bition and attention processes in ADHD to provide more insight into the core deficit in this disorder (Oosterlaan, Logan, \& Sergeant, 1998; Oosterlaan \& 
Sergeant, 1996; Plizka, Bocherding, Spratley, Leon, \& Irick, 1997; Schachar, Mota, Logan, Tannock, \& Klim, 2000; Schachar, Tannock, Mariott, \& Logan, 1995).

In contrast to the abundance of articles on inhibitory control in children with ADHD (Oosterlaan et al., 1998), far less research has been carried out in which the Stop Signal Task was administered to adults with ADHD. A limited number of studies have reported a non-significantly slower reaction time (Bekker et al., 2005; Epstein, Johnson, Varia, \& Conners, 2001) or a faster reaction time (RT) (Aron, Dowson, Sahakian, \& Robbins, 2003; P. Murphy, 2002; Ossman \& Mulligan, 2003). Also, an increase in variability in response speed (SDRT) was reported (Epstein et al., 2001) in adult patients with ADHD as opposed to healthy controls. This is suggestive of a deficiency in attention regulation (Castellanos \& Tannock, 2002). One study by Aron et al. (2003) reported that adults with ADHD made more discrimination errors (choice errors on the go task). This has been interpreted in terms of disinhibition of a premature response. Finally, all these studies, except the Epstein et al. (2001) one, have shown a slower SSRT in adults with ADHD (Aron et al., 2003; Bekker et al., 2005; Murphy, 2002; Ossman \& Mulligan, 2003; Wodushek \& Neumann, 2003). Recently, some authors have suggested in a meta-analysis that adults with ADHD have a specific deficiency in inhibitory control performance rather than a deficit in attention compared to children with ADHD (Lijfijt et al., 2005). The conclusion of these authors was largely based on the disproportionately larger deficit in SSRT compared to mean RT in unmedicated ADHD adults.

A number of studies showed a positive effect of MPH on measures of inhibitory control (SSRT), but also on measures of attention (MRT and SDRT) of the SST in children with ADHD (Hanisch, Konrad, Günther, \& Herpertz-Dahlmann, 2004; Kempton et al., 1999; Overtoom et al., 2003; Scheres et al., 2003). In adults with ADHD, effect studies of MPH by use of the SST are scarce. A study by Aron et al. (2003) revealed that MPH improved stopping (by SSRT) but not presumed measures of attention (RT and discrimination errors). This was also revealed in a study by Boonstra, Kooij, Oosterlaan, Sergeant, and Buitelaar (2005) in that there were large effects of MPH on the SSRT. In contrast, Bouffard, Hechtman, Minde, \& laboni-Kassab (2003) found no effect of MPH medication on SSRT compared to placebo in adults with ADHD. Thus, the present findings remain mixed regarding the effect of MPH on inhibition and attention processes in adults with ADHD.

Taken together, the aim of this study was to investigate whether stimulant medication MPH affects inhibitory control and measures of attention in adults with ADHD to test the hypothesis of a primary disorder in inhibitory control or a more general deficit in attention in ADHD. To this end, adults with ADHD while on and off MPH medication treatment were compared to healthy control subjects on a stop-signal task. We also examined whether performance differed within the adult ADHD group as a function of MPH treatment to test the pervasiveness of deficits in inhibitory control and/or attentional processes. For these purposes, adults with ADHD combined type (ADHD-C) were chosen as this is the most 
prevalent subtype in adult ADHD (Barkley, 1998; Manuzza, Klein, \& Moulton, 2003; K. R. Murphy, Barkley, \& Bush, 2002; Weiss \& Trokenberg-Hechtman, 1993). This choice was also made in order to make generalisations possible, as most studies are performed on this subtype. Based on previous literature, we expected a more specific inhibitory deficit in ADHD adults while not taking MPH medication. Longer mean RT, larger variability or attention lapses (SDRT) would be indicative a deficit in 'attention'. We also hypothesized that stimulant medication would improve primarily inhibitory processes in the adults with ADHD. This prediction was based on earlier studies as described in this introduction and studies that suggest that methylphenidate acts upon more frontal regions and dopaminergic systems which are known to be involved in inhibitory control (Durston, 2003).

\section{Method}

\section{Participants}

In the present study, the initial sample consisted of two groups ( $N=35)$ : 17 adults with ADHD (mean age $=28.8, S D=6.24$; range 21.9-41.9) and 18 education and age-matched normal control adults (mean age $=28.5, S D=6.44$; range 21.3 41.3). The ADHD group consisted of 16 individuals with combined attention and hyperactivity problems (ADHD-Combined) and one adult with mainly attention problems (ADHD- Inattentive). All participants were males and right-handed. Participants in the ADHD group were diagnosed by an outside clinical professional. Also, they had a minimum score of five symptoms of inattention and/or hyperactivity-impulsivity on the Current Symptoms Scale and a score of at least four symptoms of inattention and/or hyperactivity-impulsivity on the retrospective Childhood Symptoms Scale (Barkley \& Murphy, 1998). Exclusion criteria for all participants were presence of any current Axis-I psychiatric diagnosis other than ADHD, IQ $<80$, neurological trauma or disorder. An additional exclusion criterion for the control group was five or more symptoms of inattention and/ or hyperactivity on the Current Symptoms Scale and/ or Childhood Symptoms Scales. Participants of the ADHD group were recruited by spreading information brochures during group assessments of adults with ADHD and by advertisements on websites of ADHD associations. Control participants were recruited by advertisements in local news-papers in the southern parts of the Netherlands. All participants provided written informed consent for participation and received 50 euros after completion of the experiment.

Table 1 summarizes the demographic and clinical data for the ADHD-combined type patients and healthy controls included in the present study. A total of four participants were excluded. One participant with ADHD (ADHD-Inattentive) displayed not enough symptoms of ADHD on both the ADHD Rating Scales 
(maximum of three symptoms per scale). Three participants of the control group displayed too many ADHD symptoms (five or more symptoms on the current and/or childhood ADHD scale). Also, three ADHD participants that were treatment naive during this whole study and at the time of testing were excluded. Therefore, the final sample consisted of 28 participants ( $n$ ADHD $=13$; $n$ healthy controls $=15$ ). The groups did not significantly differ with regard to age, educational level, and the performance of the Block Design subtest of the Wechsler Adult Intelligence Scale, as an estimate of general abilities (Wechsler, 1981). As expected, the ADHD patients displayed significantly more current and childhood ADHD symptoms than the controls and differed from the controls in their use of MPH medication.

Table 1. Demographic data for the ADHD and control groups.

\begin{tabular}{|c|c|c|c|c|c|c|}
\hline & \multicolumn{2}{|c|}{$\begin{array}{c}\text { ADHD } \\
(n=13)\end{array}$} & \multicolumn{2}{|c|}{$\begin{array}{l}\text { Control } \\
(n=15)\end{array}$} & \multirow[b]{2}{*}{ ta } & \multirow[b]{2}{*}{$p$} \\
\hline & Mean & $S D$ & Mean & $S D$ & & \\
\hline Age & 29.9 & 6.7 & 29.4 & 6.6 & .20 & .843 \\
\hline Education level b & 4.6 & 1.6 & 5.4 & 1.4 & -1.23 & 231 \\
\hline Block Design $T$ score & 63.8 & 6.9 & 65.6 & 8.4 & -.62 & .542 \\
\hline Medication (yes/no) c & $13 / 0$ & & $0 / 15$ & & 24.27 & $<.001$ \\
\hline No. current ADHD symptoms & 13.4 & 2.0 & 0.3 & .6 & 22.31 & $<.001$ \\
\hline No. childhood ADHD symptoms & 12.8 & 4.2 & 1.0 & 1.6 & 9.34 & $<.001$ \\
\hline
\end{tabular}

Note. $\mathrm{ADHD}=$ Attention Deficit Hyperactivity Disorder. $a \mathrm{df}=26$ for age, educational level, Block Design, $d f=22$ for both ADHD symptoms. ${ }^{\circ}$ Educational level was measured on an 8point scale ranging from primary school to university degree (De Bie, 1987). cStimulant medication usage at baseline for ADHD patients versus controls was measured by use of Chi-square test. $n=10$ ADHD; short-acting methylphenidate (Ritalin $\AA$ ). $n=3$ ADHD; longacting methylphenidate (Concerta®).

\section{Procedure}

All testing was conducted in Dutch and each participant was tested individually in a special testing room. All participants were administered the same version of the Stop Signal Task during both sessions. Each session lasted two hours and they were about two weeks apart. Participants with ADHD while taking stimulant medication ( $n=13$ ) were included during the first session (baseline). In the second session (follow-up), ADHD participants were requested to refrain from their ADHD medication (MPH) at least 24 hours before testing $(n=13)$. Participants were already on medication before this study and medication dosage was established by a clinical professional based on their diagnosis of ADHD. All healthy participants were also tested twice using the same SST in each session. The SST was part of a larger neuropsychological battery including the subtest Block Design of the WAIS-R and verbal memory tests. The present study focused only on the results of the Stop Signal Tasks. After completion of the entire experiment all participants were asked to fill in the Current Symptoms Scale and Childhood Symptoms Scale. 
The study reported in the present paper was part of a larger cross-sectional study on cognitive functions in adults with ADHD in which multiple researchers participated.

Measures

ADHD Rating Scale for Adults (Barkley \& Murphy, 1998). This scale is a short, self-report screening questionnaire to assess ADHD in adults (Barkley \& Murphy, 1998; P. Murphy \& Schachar, 2000). Participants completed two versions of this scale, the Current Symptoms Scale, for current symptoms (past six months), and the Childhood Symptoms Scale, for recall of childhood symptoms between the ages 5 and 12 years (Barkley \& Murphy, 1998). Both versions contain the 18 items, including nine items measuring inattention symptoms and nine items measuring hyperactivity/impulsivity symptoms, from the diagnostic criteria for ADHD in the Diagnostic and Statistical Manual of Mental Disorders (American Psychiatric Association [APA], 1994) with a slight adaptation in word use for the adult version as a number of behaviors are not appropriate anymore (e.g., climbing). Each item is rated on a Likert scale from 0 to 3, with $0=$ not at all or rarely, 1 = sometimes, 2 = often, and 3 = very often. A score is considered a symptom if an item is answered with 'often' or 'very often', leading to a maximum of 9 symptoms for inattention and 9 symptoms for hyperactivity/impulsivity (max. total ADHD score $=18$ ).

For participants with ADHD two questionnaires of current symptoms were provided; one with symptoms of ADHD when they were taking MPH medication and one copy referring to symptoms during the treatment-naive period both consisting of the same items. For the present study, the current mean number of ADHD symptoms while on and off MPH medi-cation and their mean childhood ADHD symptoms are presented in Table 1.

The WAIS-R subtest Block Design. The subtest Block Design of the WAIS-R measures visuospatial abilities and is frequently used as an estimation of general intelligence (Wechsler, 1981; Stinissen, Willems, Coetsier, \& Hulsman, 1970). Furthermore, if matched for level of education, no difference in performance between adults with ADHD and normal control is expected (Bridgett \& Walker, 2006; Seidman, Biederman, Weber, Hatch, \& Faraone, 1998). Therefore, in the present study, where groups are matched for level of education and for age, the Block Design subtest can be used to check for similar levels of general intelligence in both groups. The block design raw scores were transformed to standard $T$ scores $(M=50 ; S D=10)$ to compare these data.

The Stop Signal Task (SST). The Stop Signal Task (G. D. Logan, 1994; G. D. Logan, Schachar, R.J., \& Tannock, R., 1997; Schachar \& Logan, 1990) is a com- 
puterized measure of inhibitory control that examines the ability to inhibit a prepotent response to a usually visual choice reaction time task in which speed and accuracy are emphasized. The Stop Signal Task consists of go and stop trials. The stimuli for the primary (go) task were the upper-case letters $O$ and $Z$ (font Arial, type size 50), presented in the center of the computer screen for a maximum of 1000 ms against a black screen and then fol-lowed by a black screen for 1000 ms. The participant's task was to identify the letter presented and to respond to the letter by pressing one of the two keys as fast and as accurate as possible. On $25 \%$ of the go trails, an auditory stop signal (i.e., a $1000 \mathrm{~Hz}$ tone generated by the computer) was presented with a duration of $100 \mathrm{~ms}$ indicating that the response to the go stimulus had to be withhold. Auditory stop signals were presented at different fixed delays (i.e., the interval between the presentation of the go trial and stop signal), namely at 50, 100, 200, and $350 \mathrm{~ms}$ after the onset of the gotask stimulus (Band, van der Molen, \& Logan, 2003; G. D. Logan, 1994; Oosterlaan \& Sergeant, 1996).

The experimental task consisted of 8 blocks of 32 trials each (i.e., a total of 256 trials), with 24 go trials and 8 stop signal trials, respectively. There was an equal number of $Z s$ and $O s$ in each block and an equal number of stop signals (i.e., two stop signals per delay) for both letters. Also, in each block two successive stop signals for a different delay respectively were at random presented. The order in which the go trials were presented was pseudorandom within each block with no more than three repetitions of the same letter in each block and this procedure was fixed for each block. If the reaction time on the go trials was longer than $600 \mathrm{~ms}$, participants received visual feedback (for $1000 \mathrm{~ms}$ ) indicating that they 'had to respond faster' on the subsequent trials. The reason for this instruction was to avoid that they would search for a strategy for waiting on the stop signal. After two blocks there was a pause for 4000 ms that was presented on the screen and this occurred three times. Participants were told that they could blink their eyes for a moment during this pause, but were also stimulated to stay alert as the pause did not last long.

The Stop Signal Task procedure was administered to each participant individually. Each participant completed two practice blocks before starting the experimental task (eight blocks). The participant was seated in front of the computer screen with left and right index fingers on two keys labelled ' $Z$ ' (left index for letter Z) and '/ '(right index for letter $O$ ). The first practice block consisted of 32 stimuli. The participants had to focus on the go task only, namely pressing the right (O) and left (Z) keys as quickly as possible and without making mistakes. In the second practice block, consisting of 32 stimuli, participants were required to practice with the stop task with stop signals at different fixed delays. Participants were instructed to continue to respond as quickly as possible without making mistakes, and trying to stop responding on the trials when they heard the tone. They were also instructed not to wait for the stop signal, because the tone occurred randomly and infrequently. This practice session was then followed by 
the experimental task. The total task including the practice sessions lasted about $16 \mathrm{~min}$.

The dependent measures for the go task were: mean RT for each block (i.e., to measure time-on task effect as a measure of sustained attention) and median RT averaged over all the blocks, the within-subject standard deviation of RT (SDRT) averaged over all the blocks, the percentage of total omissions, and the percentage of total discrimination errors. Inhibition-related dependent measures included: the percentage of successful inhibition (Pi), the delay between stimulus and the tone to yield a performance of $50 \%$ successful inhibitions (SOA; stimulus onset asynchrony), and the SSRT. The SSRT was estimated as described in G. D. Logan (1994) by subtracting the SOA from the median RT.

\section{Statistical analysis}

All data were first screened for violations of the assumptions of the parametric tests (independent $t$ - tests, GLM repeated measures analyses). Where appropriate, nonparametric statistics (Mann-Whitney signed ranked tests) were used for not normally distributed data. Participants with accuracy lower than $75 \%$ on the go trials and $0 \%$ successful inhibition on the stop trials were excluded from all analyses. This resulted in exclusion of two ADHD participants for baseline session 1 and one ADHD participant at follow-up session 2. In addition, individuals with a fit $\left(R^{2}\right)$ lower than .70 plotted over the four stop signal delays successful inhibition were excluded for the SSRT analyses, this led to the exclusion of two ADHD participants at baseline and two ADHD and one healthy control participants at follow-up. Additionnally, one participant with ADHD was excluded because of extreme values ( $>3$ time interquartile range from the mean) for the SSRT and SOA analyses for session 1 and one ADHD participant for the SOA analyses for session 2. The rejection criterion was set at $p<.05$, two-tailed. All analyses were performed using SPSS 11.5 windows package.

\section{Results}

Table 2 summarizes the results of all key variables, including t-test or MannWhitney test values and levels of significance.

Variability (SDRT): medication effect

A 2 (Session) × 2 (Group) GLM repeated measures analysis for variability on go trials (SDRT) revealed non-significant within-subject effects of Session, $F(1,23)=$ .99, $p=.328$, and Session by Group interaction, $F(1,23)=.01, p=.913$. However, $a$ significant main effect of Group was found, $F(1,23)=.01, p=.016$. Overall, ADHD adults had a larger variability in response speed on the go task compared to con-trols over both sessions, irrespective of medication use (as can be also seen in Table 2 for both sessions separately). 
RT, SOA, and SSRT: medication effect

A 2 (Test: median RT vs. SOA) × 2 (Session) × 2 (Group) GLM repeated measures analysis revealed a significant effect of Test, $F(1,19)=569.3, p<.001$, a significant Test by Group interaction, $F(1,19)=6.906, p=.017$, and a significant three way Test by Session by Group interaction, $F(1,19)=6.87, p=.017$ indicating that the different performance measures were not parallel for both groups across the two sessions. The Test by Group interaction indicated that the ADHD adults had overall a longer go-stop interval (SOA), but a shorter median RT across both sessions compared with healthy controls. Post-hoc paired samples t-tests revealed that this SOA to obtain successful inhibition decreased from session 1 to session 2 within the ADHD group, although this was not statistically significant ( $p=.469$ ). While the SOA for successful inhibition increased from session 1 to 2 , this effect was also not significant $(p=.665)$. We did find that the ADHD adults exhibited a lower go-stop interval on both sessions compared to healthy controls (see Table 2). Regarding the median RT, we found no significant Session effect of medication within the adult ADHD group $(p=.101)$ by use of paired samples $t$ tests, whereas the healthy control group became significantly faster from baseline session to follow-up session ( $p=.016$ ). Furthermore, as can be seen in Table $2, t$ tests revealed that ADHD adults showed faster median RT than healthy controls. Also, the post-hoc paired samples t-tests for MRT revealed that both within the ADHD adults $(p=.056)$ and healthy controls $(p=.046)$ became faster from baseline to follow-up.

The healthy control subjects had stop percentages (P[i]) around $60 \%$ for both sessions, indicating that the fixed stop signal delay was successfully applied. However, the ADHD adults showed significantly lower stop percentages (average $=36 \%$ ) compared to controls on both sessions (see Table 2), rather indicating their problems in inhibition.

Furthermore, our 2 (Session) × 2 (Group) GLM repeated measures analysis for performance on SSRT performance revealed no significant Session effect, $F(1$, $19)=3.52, p=.076$, but a significant Session by Group interaction, $F(1,19)=6.87$, $p=.018$. This interaction indicated that SSRT increased over session in the ADHD group, while in the control group SSRT decreased over time. A significant main effect of Group, $F(1,19)=6.90, p=.017$, indicated that overall, the ADHD adults had larger SSRT than controls. Post-hoc paired samples t- tests revealed no significant difference in SSRT over sessions within the ADHD group ( $p=.551)$, whereas in the healthy group SSRT became faster from session 1 to 2 ( $p<.001$ ).

To investigate whether the speed of responding on the go task remained stable in each group, a GLM repeated measures analysis was carried out, with group as the between-subject variable and the MRT for the eight blocks as dependent variables (Time-on task). The results showed no significant Time-on task effect and no Time-on task by Group interactions for both sessions (all ps > .09). 
Table 2. Stop signal performance data for baseline and follow-up for the ADHD and control groups

\begin{tabular}{|c|c|c|c|c|c|c|}
\hline \multirow[b]{2}{*}{ Variable } & \multicolumn{2}{|c|}{ ADHD } & \multicolumn{2}{|c|}{ Control } & \multirow[b]{2}{*}{$t / x^{2}$} & \multirow[b]{2}{*}{$p$} \\
\hline & Mean & SD & Mean & SD & & \\
\hline \multicolumn{7}{|l|}{$\begin{array}{l}\text { SST (baseline; session 1) with } \\
\text { medication }\end{array}$} \\
\hline \multicolumn{7}{|l|}{ Go task } \\
\hline Median RT & 386.5 & 36.4 & 424.6 & 48.5 & -2.18 & .039 \\
\hline MRT & 400.3 & 40.6 & 433.9 & 50.9 & -1.80 & .084 \\
\hline SDRT & 99.7 & 14.9 & 88.4 & 13.1 & 2.04 & .052 \\
\hline P(choice error) (Whitney U test) & 3.6 & 2.3 & 3.1 & 3.5 & 61.5 & .273 \\
\hline P(omission) (Whitney U test) & .1 & .2 & .5 & 1.0 & 73.0 & .544 \\
\hline \multicolumn{7}{|l|}{ Stop task } \\
\hline $\mathrm{P}(\mathrm{i})(\%)$ & 38.3 & 17.5 & 58.9 & 15.5 & -3.16 & .004 \\
\hline SOA & 137.5 & 70.6 & 207.7 & 63.4 & -2.51 & .020 \\
\hline SSRT & 247.6 & 45.8 & 216.8 & 40.1 & 1.72 & .098 \\
\hline \multicolumn{7}{|l|}{$\begin{array}{l}\text { SST (follow-up; session 2) without } \\
\text { medication }\end{array}$} \\
\hline \multicolumn{7}{|l|}{ Go task } \\
\hline Median RT & 368.9 & 52.4 & 405.7 & 59.3 & -1.66 & .109 \\
\hline MRT & 377.6 & 55.0 & 418.4 & 59.7 & -1.80 & .084 \\
\hline SDRT & 101.6 & 19.6 & 85.3 & 10.6 & 2.68 & .013 \\
\hline P(choice error) (Whitney U test) & 7.5 & 6.3 & 2.9 & 3.1 & 33.50 & .009 \\
\hline P(omission) (Whitney U test) & .5 & .7 & .1 & .4 & 53.50 & .054 \\
\hline \multicolumn{7}{|l|}{ Stop task } \\
\hline $\mathrm{P}(\mathrm{i})(\%)$ & 33.7 & 21.5 & 60.0 & 15.9 & -3.56 & .002 \\
\hline SOA & 121.1 & 96.5 & 222.3 & 61.3 & -3.01 & .007 \\
\hline SSRT & 263.1 & 62.1 & 187.6 & 36.3 & 3.27 & .007 \\
\hline
\end{tabular}

Note. $A D H D=$ Attention Deficit Hyperactivity Disorder. SST = Stop Signal Task. MRT = mean reaction time. SDRT $=$ within-subject standard deviation of mean reaction time. $\mathrm{P}$ (choice error $)=$ percentage of total choice errors. $\mathrm{P}$ (omission $)=$ percentage of total omissions. $\mathrm{P}(\mathrm{i})=$ percentage of inhibition averaged over four stop signal delays. SSRT = stop signal reaction time. $\mathrm{SOA}=$ stimulus onset asynchrony for $50 \%$ of inhibition.

Error analysis of go task

The 2 (Session) × 2 (Group) GLM repeated measures analysis for choice error rate revealed a significant Session by Group interaction, $F(1,23)=6.68, p=.017$, indi- 
cating that choice error rate increased over session in ADHD adults and decreased for healthy controls (see also Table 2). No significant effect for Session was found, $F(1,23)=.53, p=.805$. Post-hoc paired samples $t$ - tests also revealed that this increase in choice error rate over sessions for ADHD adults was statistically significant ( $p=.015)$, whereas the decrease for healthy controls was not statistically significant $(p=.704)$.

Lastly, the 2 (Session) $\times 2$ (Group) GLM repeated measures analysis for omissions revealed a significant Session by Group interaction, $F(1,23)=5.82, p=$ .024, indicating that omission rate increased over sessions in the ADHD adults while the reverse pattern was found for the healthy controls (as can be seen in Table 2). Post-hoc paired samples $t$ - tests also revealed this increase in omission rate from session 1 to 2 within the adult ADHD group was not statistically significant ( $p=.237$ ), whereas the decrease in omission rate for healthy controls appeared statistically significant $(p=.043)$.

\section{Discussion}

The aim of the present study was to examine the efficacy of stimulant medication (methylphenidate) on inhibition and attentional processes in adults with ADHD while on and off medication and relative to healthy controls. The present data showed deficits in inhibitory control (SSRT, SOA, choice error rate) and attentional processing (SDRT, omissions) in adults with ADHD while off MPH compared to healthy controls. MPH medication improved choice error rate, but no other measures of inhibitory control or attention in the ADHD adults.

Firstly, the present study found support for the hypothesis of a more general inattention deficit in ADHD (Castellanos \& Tannock, 2002). We found deficits on measures of inhibitory control and some measures of attention in adults with ADHD while off MPH in comparison to healthy controls. Moreover, our data revealed a significantly larger inter-trial variability, longer stop signal reaction times, and more choice errors in these adult ADHD patients. In addition, the ADHD patients off medication showed a tendency towards making more omission errors than healthy controls. This pattern of deficits is therefore rather an extension to what has been reported in children with ADHD (Lijfijt et al., 2005; Oosterlaan et al., 1998) in contrast to the suggested specific inhibitory control deficit (Barkley, 1998; Lijfijt et al., 2005) in adult ADHD. However, we found no slower speed of responding on go stimuli (MRT or median RT), which has also been reported in previous studies in adults with ADHD (Aron et al., 2003; Murphy, 2002; Ossman \& Mulligan, 2003). This might be explained by the fact that adults with ADHD were already on stimulant medication before participating in this study influencing their speed of responding and thus being better able to compensate for their processing speed. 
Secondly, our hypothesis regarding the efficacy of MPH medication on inhibition and attention measures within the adult ADHD group was only partly supported: MPH only ameliorated the choice error rate in the medicated ADHD adults compared to when not taking MPH. This can be regarded as evidence for improvement in the premature or impulsive responding in the ADHD adults. Furthermore, no other effect of MPH medication was found for response variability (SDRT), response speed (median RT), SSRT, SOA or omissions when comparing ADHD adults on and off medication. This finding is in line with the Bouffard et al. (2003) study who found no effect of MPH on SSRT in ADHD adults compared to placebo. These results are however not consistent with the Aron et al. (2003) study who reported an improvement in SSRT, but not in go RT and choi-ce errors in the medicated ADHD adults. Our results further indicate that the variability in response speed remains a deficit in the adults with ADHD, irrespective of MPH treatment. Moreover, the ADHD adults displayed higher response variability on both sessions compared to healthy controls. This is also in line with Douglas (1999) who indicated that inconsistency in responding likely represents a pervasive impairment. But also confirms a recent statement by Castellanos \& Tannock (2002) suggesting that "the transient but frequent lapses of intention and attention, and the moment-to-moment variability and inconsistency are the most striking clinical characteristics of ADHD" (p.624).

Furthermore, our results regarding the comparison of median RT and gostop interval for successful inhibition (SOA) merit further explanation. Moreover, we found that while both ADHD and healthy control groups showed faster reaction time on the go task from baseline to follow-up session, which was not significant for the ADHD adults, the ADHD adults remained faster in their RT compared to healthy controls when they were on MPH medication. We chose to compare their median RT as this was also used in the calculation of the SSRT and as more positive skewness has been reported in ADHD in which the median RT is preferred to be used (Leth-Steensen, King Elbaz, \& Douglas, 2000). Also, our data revealed that go-stop interval for successful inhibition (SOA) did not differ over both sessions within the ADHD adults, although increased when on MPH medication. However, these ADHD adults had longer SOAs compared to healthy controls on both sessions. These results suggest that the ADHD adults may adopt a less efficient coping strategy, in that they generally respond faster on the go trials than controls and thus are more likely to have more difficulty in inhibiting a prepotent response. In contrast, the healthy controls possibly adopt a more efficient strategy, in that their RT is somewhat slower, even though faster over time sessions, than the ADHD adults. This suggests that the healthy controls are better capable of inhibiting their prepotent response compared to ADHD adults. Indeed, we also found that the ADHD adults exhibited a longer SSRT compared to healthy controls over both sessions which was also supported by no medication effect on SSRT within the ADHD adults. Therefore, we contend that ADHD adults have a deficit in inhibitory control that seems to be mediated 
by a more general deficit in attention lapses (Castellanos \& Tannock, 2002; Douglas, 1999). Their pattern of responding (slower SSRT, higher variability in speed) may rather result from a non-optimal activation or effort state as has been suggested in children with ADHD (Kuntsi et al., 2001; Oosterlaan \& Sergeant, 1996; Van der Meere \& Sergeant, 1988).

Likewise, our findings showed that methylphenidate treatment resulted in a lower amount of choice errors in the adult ADHD patients. This has also been revealed by Aron et al. (2003), suggesting an improvement in premature or impulsive responding. Therefore, our results regarding the efficacy of MPH treatment provide support for the notion that MPH act more strongly on inhibitory control processes and less on attention in our adult ADHD patients. This is in agreement with what has been reported in a clinical study on MPH in children with ADHD (Lijffijt et al., 2006). This dissociation may be caused by differing dopaminergic and norepinephrinergic effects of MPH on the orienting response (Lijffijt et al., 2006).

A limitation of the present study is the relatively small sample size, which may have reduced the statistical power. Replication in a larger ADHD sample can shed more light on the effects of SOA compared to median RT over medication sessions. It may be that the reverse pattern in the ADHD adults relative to the healthy controls reveals significant results, indicating an inhibition deficit in ADHD adults.

The present results have also several implications. Our findings indicate that the Stop Signal Task is a sensitive test to detect differences in inhibition and attention processes in adults with ADHD relative to healthy controls. We therefore suggest that clinical settings could benefit from using this task in the assessment of ADHD. A pattern of slower SSRT and increased inconsistency can be more likely apparent in the ADHD combined type. Future studies extending these findings to ADHD subtypes as to whether specific profiles in inhibition and attention processes can be differentiated is warranted. Of all task variables, variability in speed of responding was found to best useful in distinguishing ADHD adults when on and of $\mathrm{MPH}$ from healthy participants. Clinicians should be more alert on this important characteristic when testing patients for an ADHD diagnosis. Future normative data of healthy and ADHD participants on the presented SST variables for the Dutch speaking population are necessary to implement this task in the clinic. Furthermore, our results indicate that ADHD adults benefit from taking MPH medication particularly in their impulsive responding and accuracy. This has major direct implications on their daily life activities, i.e., in their occupation where these adults may be capable in controlling themselves and their activities, being less error prone and eventually create a greater satisfaction. Future research needs to confirm these hypotheses. 


\section{Acknowledgement}

We thank Katya Bakker for her major role in test assistance for this project. We also thank all those who kindly volunteered to participate in this study. 


\section{References}

American Psychiatric Association (2000). Diagnostic and Statistical Manual of Mental Disorders: DSM-IV-TR. Washington, DC: American Psychiatric Press.

Aron, A. R., Dowson, J. H., Sahakian, B. J., \& Robbins, T. W. (2003). Methylphenidate improves response inhibition in adults with Attention-Deficit/Hyperactivity Disorder. Biological Psychiatry, 54, 1465-1468.

Band, G. P. H., van der Molen, M. W., \& Logan, G. D. (2003). Horse-race model simulations of the stop-signal procedure. Acta Psychologica, 112, 105-142.

Barkley, R. A. (1997). Behavioral inhibitions, sustained attention, and executive functions: constructing a unifying theory of ADHD. Psychological Bulletin, 121, 65-94.

Barkley, R. A. (1998). Attention-Deficit/ Hyperactivity Disorder: A handbook for diagnosis and treatment (2nd ed.). New York: The Guilford Press.

Barkley, R. A., \& Murphy, K. R. (1998). Attention deficit hyperactivity disorder: A clinical workbook. New York: Guilford.

Bekker, E. M., Overtoom, C. C., Kenemans, J. L., Kooij, J. J., De Noord, I., Buitelaar, J. K., et al. (2005). Stopping and changing in adults with ADHD. Psychological Medicine, 35, 807-816.

Biederman, J., Faraone, S. V., Taylor, A., Sienna, M., Williamson, S., \& Fine, C. (1998). Diagnostic continuity between child and adolescent ADHD: Findings from a longitudinal clinical sample. Journal of the American Academy Child and Adolescence Psychiatry, 37(30), 305-313.

Boonstra, A. M., Kooij, J. J. S., Oosterlaan, J., Sergeant, J. A., \& Buitelaar, J. K. (2005). Does methylphenidate improve inhibition and other cognitive abilities in adults with childhood-onset ADHD? Journal of Clinical and Experimental Neuropsychology, 27, 278-298.

Bouffard, R., Hechtman, L., Minde, K., \& laboni-Kassab, F. (2003). The efficacy of 2 different dosages of methylphenidate in treating adults with attention-deficit hyperactivity disoder. canadian Journal of Psychiatry, 49, 557-568.

Bridgett, D. J., \& Walker, M. E. (2006). Intellectual functioning in adults with ADHD: A metaanalytic examination of full scale IQ differences between adults with and without ADHD. Psychological Assessment, 18(1), 1-14.

Castellanos, F. X., \& Tannock, R. (2002). Neuroscience of attention-deficit/hyperactivity disorder: the search for endophenotypes. Nature Reviews Neuroscience, 3, 617628.

De Bie, S. E. (1987). Standaardvragen 1987: Voorstellen voor uniformering van vraagstellingen naar achtergrondkenmerken en interviews [Standard questions 1987: Proposal for uniformisation of questions regarding background variables and interviews] (2nd ed.). Leiden, the Netherlands: Leiden University Press.

Douglas, V. I. (1999). Cognitive control processes in attention deficit hyperactivity disorder. In H. C. Quay \& A. E. Hogan (Eds.), Handbook of Disruptive Behavior Disorders (pp. 105-138). New York: Kluwer Academic / Plenum Publishers.

Durston, S. (2003). A review of the biological bases of ADHD: What have we learned from imaging studies. Mental Retardation and Developmental Disabilities, 9, 184-195. 
Epstein, J. N., Johnson, D. E., Varia, I. M., \& Conners, C. K. (2001). Neuropsychological assessment of response inhibition in adults with ADHD. Journal of Clinical and Experimental Neuropsychology, 23, 362-371.

Hanisch, C., Konrad, K., Günther, T., \& Herpertz-Dahlmann, B. (2004). Age-dependent neuropsychological deficits and effects of methylphenidate in children with attention-deficit/hyperactivity disorder: A comparison of pre- and grade-school children. Journal of Neural Transmission, 111, 865-881.

Hervey, A. S., Epstein, J. N., \& Curry, J. F. (2004). Neuropsychology of adults with AttentionDeficit/Hyperactivity Disorder: A meta-analytic review. Neuropsychology, 18(3), 485-503.

Kempton, S., Vance, A., Maruff, P., Luk, E., Costin, J., \& Pantelis, C. (1999). Executive function and attention deficit hyperactivity disorder: stimulant medication and better executive function performance in children. Psychological Medicine, 29, 527-538.

Kuntsi, J., Oosterlaan, J., \& Stevenson, J. (2001). Psychological mechanisms in hyperactivity: I Response inhibition deficit, working memory impairment, delay aversion, or something else? Journal of Child Psychology and Psychiatry, 42 (2), 199-210.

Leth-Steensen, C., King Elbaz, Z., \& Douglas, V. I. (2000). Mean response times, variability, and skew in the responding of ADHD children: a response time distributional approach. Acta Psychologica, 104, 167-190.

Lijfijt, M., Kenemans, J. L., ter Wal, A., Quik, E. H., Kemner, C., Westenberg, H., et al. (2006). Dose-related effect of methylphenidate on stopping and changing in children with attention-deficit/hyperactivity disorder. European Psychiatry, 21, 544-547.

Lijffijt, M., Kenemans, J. L., Verbaten, M. N., \& van Engeland, H. (2005). A meta-analytic review of stopping performance in attention-deficit/hyperactivity disorder: Deficient inhibitory motor control? Journal of Abnormal Psychology, 114(2), 216222.

Logan, G. D. (1994). On the ability to inhibit thought and action. A user's guide to the stopsignal paradigm. San Diego: Academic Press.

Logan, G. D., Schachar, R.J., \& Tannock, R. (1997). Impulsivity and inhibitory control. Psychological Science., 8(1), 60-64.

Manuzza, S., \& Klein, R. G. (2000). Long-term prognosis in attention-deficit/ hyperactivity disorder. Child and Adolescent Psychiatric Clinics of North America, 9(3), 71 1-726.

Manuzza, S., Klein, R. G., \& Moulton, J. L. (2003). Persistence of Attention-Deficit/Hyperactivity Disorder into adulthood: What have we learned from the prospective followup studies? Journal of Attention Disorders, 7(2), 93-100.

Murphy, K. R., Barkley, R. A., \& Bush, T. (2002). Young adults with Attention Deficit Hyperactivity Disorder: Subtype differences in comorbidity, educational, and clinical history. The Journal of Nervous and Mental Disease, 190(3), 147-157.

Murphy, P. (2002). Cognitive functioning in adults with Attention-Deficit/Hyperactivity Disorder. Journal of Attention Disorders, 5(4), 203-209.

Murphy, P., \& Schachar, R. (2000). Use of self-ratings in the assessment of symptoms of Atten-tion Deficit Hyperactivity Disorder in adults. American Journal of Psychiatry, 157, 1156-1159.

Oosterlaan, J., Logan, G. D., \& Sergeant, J. A. (1998). Response inhibition in AD/HD, CD, comorbid $A D / H D$, anxious, and control children: a meta analysis of studies with the stop task. Journal of Child Psychology and Psychiatry, 39, 41 1-425. 
Oosterlaan, J., \& Sergeant, J. A. (1996). Inhibition in ADHD, agressive, and anxious children: A biologically based model of child psychopathology. Journal of Abnormal Child Psychology, 24, 19-36.

Ossman, J. M., \& Mulligan, N. W. (2003). Inhibition and attention deficit hyperactivity disorder in adults. American Journal of Psychology, 116(1), 35-50.

Overtoom, C. C. E., Verbaten, M. N., Kemner, C., Kenemans, J. L., van Engeland, H., Buitelaar, J. K., et al. (2003). Effects of methylphenidate, desipramine, and L-dopa on attention and inhibition in children with attention deficit hyperactivity disorder. Behavioral Brain Research, 145, 7-15.

Plizka, S. R., Bocherding, S. H., Spratley, K., Leon, S., \& Irick, S. (1997). Measuring inhibitory control in children. Developmental and Behavioral Pediatrics, 18(4), 254-259.

Quay, H. C. (1997). Inhibition and Attention Deficit Hyperactivity Disorder. Journal of Abnormal Child Psychology, 25(1), 7-13.

Schachar, R., \& Logan, G. D. (1990). Impulsivity and inhibitory control in normal development and childhood pathology. Developmental Psychology, 26(3), 710-720.

Schachar, R., Mota, V. L., Logan, G. D., Tannock, R., \& Klim, P. (2000). Confirmation of an inhibitory control deficit in attention-deficit/hyperactivity disorder. Journal of Abnormal Psychology, 28(3), 227-235.

Schachar, R., Tannock, R., Mariott, M., \& Logan, G. (1995). Deficient inhibitory control in attention deficit hyperactivity disorder. Journal of Abnormal Child Psychology.

Scheres, A., Oosterlaan, J., Swanson, J., Morein-Zamir, S., Meiran, N., Schut, H., et al. (2003). The effect of methylphenidate on three forms of response inhibition in boys with AD/HD. Journal of Abnormal Child Psychology, 31 (1), 105-120.

Seidman, L. J., Biederman, J., Weber, W., Hatch, M., \& Faraone, S. V. (1998). Neuropsychological functioning in adults with attention-deficit hyperactivity disorder. Biological Psychiatry, 44, 260-268.

Stinissen, J., Willems, P. J., Coetsier, P., \& Hulsman, W. L. L. (1970). Handleiding bij de Nederlandstalige bewerking van de Wechsler Adult Intelligence Scale (WAIS). Lisse, Netherlands: Swets \& Zeitlinger.

Van der Meere, J., \& Sergeant, J. (1988). Controlled processing and vigilance in hyperactivity: Time will tell. Journal of Abnormal Child Psychology, 16(6), 641-655.

Weiss, G., \& Trokenberg-Hechtman, L. (1993). Hyperactive children grown up. New York: The Guilford Press.

Wilens, T. E., Spencer, T. J., \& Biederman, J. (2002). A review of pharmacotherapy of adults with attention-deficit/hyperactivity disorder. Journal of Attention Disorders, 5, 189202.

Wodushek, T. R., \& Neumann, C. S. (2003). Inhibitory capacity in adults with symptoms of Attention Deficit/Hyperactivity Disorder (ADHD). Archives of Clinical Neuropsychology, 18, 317-330. 


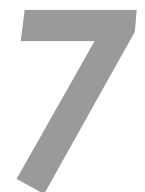

Concluding Remarks 
138 | Chapter 7 
ADHD is a serious and pervasive neurodevelopmental disorder that can affect an individual from childhood to adulthood. We now know from the childhood studies that this syndrome is characterized by multiple cognitive deficits, such as problems on the domain of executive functioning. Since this disorder also persists into adulthood, it is imperative to understand whether a similar cognitive profile in adults with ADHD occurs. By examining this, we will be able to gain more insight into the underlying nature and expression of behavioral symptoms in adults with ADHD and whether there is any change in cognitive abilities in these ADHD adults.

The main objective of this dissertation was to disentangle the more complex cognitive processes, notably executive functions, underlying standard test performance in adults with ADHD. The term 'executive function'(EF) has been conceptualised as an umbrella term for the inter-related higher-order cognitive processes responsible for goal-directed and contextually appropriate behavior (Alvarez \& Emory, 2006; Anderson, 2002; Lezak, 1995; B.F. Pennington \& Ozonoff, 1996; Spreen \& Strauss, 1998). Although many aspects of cognitive functioning can be studied, the complex functions described in the present dissertation were chosen based on research findings in children with ADHD and for their importance in daily life of individuals with ADHD as reported in clinical practice. In order to achieve this, the studies described in this dissertation investigated a number of issues that arise when drawing conclusions on cognitive dysfunctioning in adult ADHD. The first issue was the impact of comorbidity on our results and thus the specificity of cognitive dysfunctions and processes in adults with ADHD. The second issue that we addressed was whether ADHD subtypes can be differentiated from each other by use of alternative measures for cognitive processes underlying their learning performance. The third and final issue that we examined was whether methylphenidate can be used as a tool to further explore these underlying cognitive processes in adult ADHD.

The results of the cross-sectional studies performed to provide more insight into these questions were reported in this dissertation. Each of these studies focused on one or more distinct cognitive functions and processes in adults diagnosed with ADHD (combined or inattentive subtype), but also in adults with ADHD and one or more comorbid disorders, adults with other psychopathology than ADHD, and healthy control adults. The age range in the studies varied from 18 to 61 years. Adults over the age of 61 were not included because of the known age-related cognitive deterioration after 60 years and the influence of (pre-) retirement (Houx, \& Jolles, 1993; Houx, Vreeling, \& Jolles 1991; Salthouse, 1996). In this chapter, the general findings of the neuropsychological studies described in this dissertation are discussed in the light of the objectives of this dissertation and its theoretical context. Moreover, the value of cognitive research in ADHD adults reported in this dissertation is provided. This chapter ends with some implications for further research and potential applications. 


\section{Executive function deficits and process approach in adults with ADHD}

The central goal of this dissertation was to investigate the more complex cognitive processes, namely executive functioning, underlying test performance in different groups of adults with ADHD. This kind of cognitive research adds to the current knowledge of adult ADHD and is new in this field.

Firstly, relatively new approaches/strategies, including response variability, information processing strategies, and memory organisational strategies, for analysing the test data were applied in adults diagnosed with ADHD. The reason for using these approaches was to gain more insight into the cognitive processes underlying standard test performance in different groups of ADHD adults. Also, these so-called process approaches were not only used in traditional cognitive tasks (Chapter 5 and 6), but also in computerised attention and information processing reaction time tests (Chapter 3 and 6). By using a process measure approach in more complex attention and information processing and inhibition tasks, it could be demonstrated that the adults with ADHD exhibited primarily a higher variability in response speed over time compared to healthy controls (Chapter 3 and 6). This result is in line with research on school-age children with ADHD (Börger et al., 1999; Castellanos \& Tannock, 2002; Kalff et al., 2003; Kuntsi, Oosterlaan, \& Stevenson, 2001) and extends to adults with ADHD. This finding indicates that this pattern of enlarged variability remains a pervasive and persistent impairment in adult ADHD. Moreover, we contend that the reported transient but frequent lapses of intention and attention reflect the moment-tomoment variability and inconsistency impairments in adults with ADHD. This variability in turn has been suggested to be an important endophenotype of ADHD (Castellanos \& Tannock, 2002) which can be extended to adults with ADHD. Therefore, our findings of persistent response variability suggest the need for careful taking note of variable performance test scores during neuropsychological assessment of adults with ADHD. This underlying enlarged variability in ADHD adults might also explain the inconsistent research results across the studies on adult ADHD that was not addressed in most of these studies. Finally, this larger intra-trial variability may play a potential role in explaining the absence of more general EF weakness, suggesting that these weaknesses are neither necessary nor sufficient to cause all cases of ADHD (Willcutt, Doyle, Nigg, Faraone, \& Pennington, 2005).

Secondly, the verbal fluency tasks which are commonly used in clinical and experimental settings including the adult ADHD population were re-analysed by use of a novel process approach (Chapter 5). The two most common types of fluency tasks are initial letter fluency and semantic category fluency (Lezak, 1995). It has been suggested that the total number of words generally produced in $60 \mathrm{~s}$ does not provide enough information about the mechanisms underlying 
verbal fluency performance (Hurks et al., 2004; A.K. Troyer, 2000). In contrast, they have indicated that the pattern of words produced over time (defined in quar-tiles of $15 \mathrm{~s}$ ) provides more insight into automatic (first $15 \mathrm{~s}$ ) and controlled cog-nitive processing (the following $45 \mathrm{~s}$ ). The data on both fluency tasks as reported in Chapter 5, revealed that the ADHD adults, though they were taking stimulant medication, could not be differentiated from the healthy controls in terms of the total number of words generated in 60 seconds on either type of fluency. This was also revealed in Chapter 2, but only drawing the same conclusions regarding semantic category fluency in unmedicated ADHD adults. On the other hand, the study presented in Chapter 5 showed that the medicated ADHD adults produced a significantly lower number of words during the first $15 \mathrm{~s}$ as compared to healthy controls on semantic category fluency. They could only be discriminated from the healthy controls on this fluency measure of more acquired automatic information processing of semantic knowledge and not so much on controlled processing on both types of verbal fluency even though they were on stimulant medication. These findings emphasize the importance of using more process measures, such as word productivity over time that is a more sensitive measure for verbal fluency performance in ADHD adults, in particular in detecting disturbances on a semantic category fluency task. Based on these results, we can also say that adults with ADHD have more problems in automatically searching through their long-term memory for semantic associations and knowledge. Thus, they need more time and effort to start their engine searching for this semantic information. In addition, the neuropsychological findings reported in the present dissertation in adults with ADHD did not extend to the all cognitive impairments revealed in children with ADHD. For instance, we found no deficit in interference control in the ADHD adults which has been commonly reported in children with ADHD (Seidman, 2006). This result reflects an underlying brain abnormality that endures in both children and adults with ADHD (Durston, 2003; Seidman, 2006) and impairs a number of cognitive functions from childhood to adulthood. This provides support for the persistence of behavioral symptoms of ADHD in adults with ADHD. On the other hand, this result suggests that through the years these ADHD adults have learned to compensate for some cognitive weaknesses, such as finding another way to neglect irrelevant information in their environment. Indeed, it has been reported that hyperactivity and impulsivity declines with increasing age while symptoms of inattention remain a core problem (Faraone, Biederman, \& Mick, 2005).

Furthermore, the results of the studies described in this dissertation contribute to elaboration of the theoretical context of EF in adults with ADHD. We used the EF model proposed by Peter Anderson (2002) as the building block to incorporate the $\mathrm{EF}$ and cognitive processes described in the present dissertation and to evaluate this model in ADHD adults. This model of EF has been described in detail in the introduction of the dissertation. Briefly, in this model, EF is conceptu- 
alised as four distinct cognitive domains, namely attentional control, information processing, cognitive flexibility, and goal setting that operate in an integrative manner (Anderson, 2002). In order to get a better picture of which EF domains are impaired in the ADHD adults, the results of the present dissertation are placed in this model (see Figure 1). According to this model of cognitive development, the first domain attentional control develops most early during infancy and early childhood and is established fully during mid-childhood. The other three domains follow different trajectories that reach full establishment by mid-adolescence. Our results from the various studies described in this dissertation demonstrate that the deficient development of these higher-order executive functions apparent from childhood in individuals with ADHD (Hurks et al., 2004; Kalff, 2002) clearly leaves it marks in adult lives of these individuals. Moreover, the results of this dissertation show that the adults with ADHD have deficiencies on all the four EF domains, but not in all processes within these domains (see Figure 1; Chapter 2 to 6). We contend that these adults with ADHD have a more general problem in deploying efficient strategies to deal with new information and have less access to a greater repertoire of strategies in decision making and learning. This is believed to be coming forth from their deficits in attentional control processes, including selective attention to stimuli, inhibition of prepotent responses or impulsiveness, detecting error or their self-monitoring that are already apparent from their childhood. Thus, we suggest that a deficit in higher-order cognitive processes is largely due to inefficient development of lower EF domains presumably from their childhood on. According to Barkley (1997; 1998), behavioral inhibition is regarded as a 'core' deficit in children with ADHD that, in turn, causes more general weaknesses in EF (i.e., working memory, self regulation of affectmotivation-arousal, internalisation of speech, and reconstitution of internally represented information). These inhibition deficits from childhood in turn may lead that these adults do not have developed appropriate coping mechanisms to deal with these failures. As a result, this can explain the common complaints, such as frequent job changes, not knowing how to prioritize or organize their work and/or housekeeping, reported by ADHD adults when referred to clinics.

Our findings also contribute to the Dual Pathway hypothesis (B. F. Pennington, 2005; Sonuga-Barke, 2005). This model purports that, in ADHD, separate pathways exist for the cognitive deficits and motivational deficits. In this model, cognitive deficits, in particular EF, are related to one frontal-striatal circuit and motivational deficits are linked to another frontal-striatal circuit. The results described in this dissertation demonstrated deficits in EF (Chapter 2 to 6), such as verbal working memory, concept shifting, motor inhibition, providing support for the cognitive pathway in ADHD adults. In addition, our results regarding a general larger variability (Chapter 3 and 6) provide support for a deficiency in the allocation of effort and attention which are regarded as motivational processes (e.g., 'state factors') (Sergeant, 2005). We therefore, tentatively suggest that 
both these pathways need to be integrated in the individual with ADHD to understand the nature of its cognitive deficits.

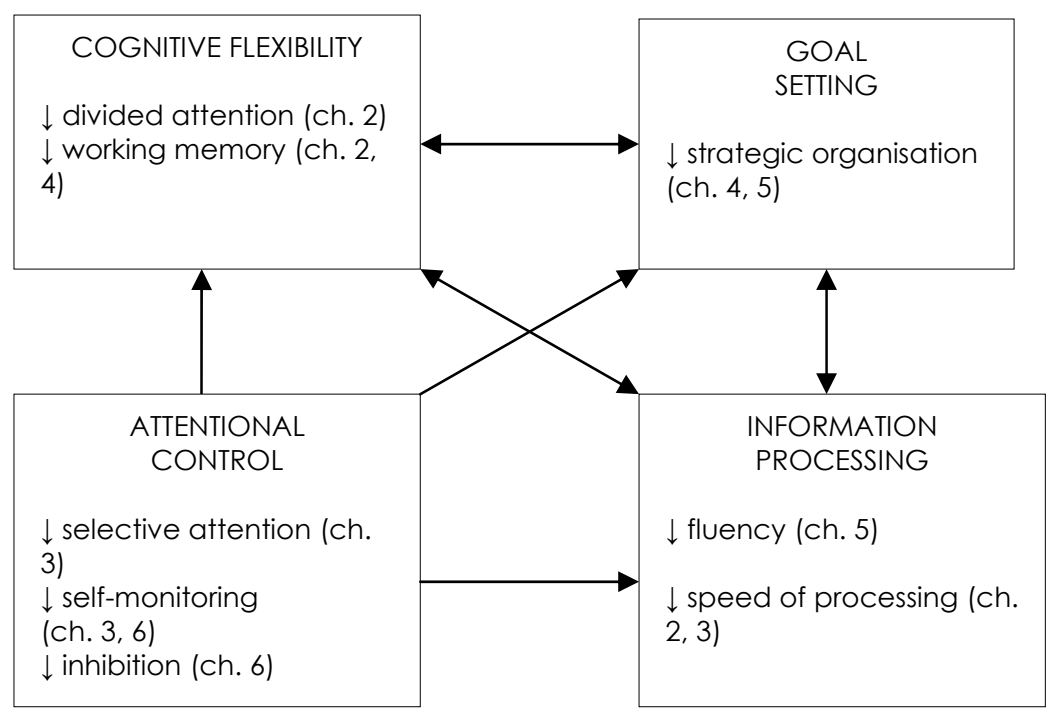

Figure 1. Results of the present dissertation placed in Peter Anderson's poposed model of executive function (2002)

(source: Anderson, P. (2002), Child Neuropsychology, 8, p. 73)

Note. Ch. $=$ referring to the chapters of this dissertation

\section{Specificity of the findings: Comorbidity and ADHD subtypes}

Another topic that added important value to the cognitive research in the field of adult ADHD described in the present dissertation was the investigation of the specificity of the impairments in the more complex cognitive processes in adults with ADHD. This concept of specificity of findings has been recognized by many (e.g., in reviews of Hervey, Epstein, \& Curry, 2004; Seidman, 2006; Woods, Lovejoy, \& Ball, 2002), but relatively little research has been conducted on this field in adults with ADHD. This is unfortunate, as it would make it possible to draw conclusions about whether the cognitive weaknesses and strengths in ADHD adults are specific for this disorder. Therefore, this topic was addressed in two important ways, namely (1) by examining the impact of comorbidity and (2) by comparing adults with a different kind of ADHD subtype (combined versus inattentive subtype) in the present dissertation. These approaches are promising and relatively new in this field. The results on these two approaches presented in this dissertation will be discussed in more detail in the following paragraphs.

Firstly, we know as mentioned in the introduction that comorbidity is an important issue to consider when examining adults with ADHD. It is rather the rule 
than the exception that adults ADHD have a high rate, varying between $10 \%$ and $70 \%$, of comorbid psychiatric conditions and/or cognitive disorders, such as difficulties in learning and reading (R.A. Barkley, Murphy, \& Kwasnik, 1996; Marks, Newcorn, \& Halperin, 2001; Milberger, Biederman, Faraone, Murphy, \& Tsuang, 1995; Millstein, Wilens, Biederman, \& Spencer, 1997). Also, in the present dissertation about the half of our ADHD population exhibited one or more comorbid disorders (Chapters 2, 3, and 4). Therefore, it is important to draw conclusions on whether the deficits in complex cognitive processes are specific for adult ADHD or rather due to the presence of comorbidity. For this purpose, we used an approach in which both adults diagnosed with ADHD and one or more comorbid disorders (the ADHD + comorbidity group), and a clinical control group of adults who did not have an ADHD diagnosis but had been diagnosed with one or more other psychiatric disorders (the non-ADHD pathological control group), and healthy control adults were compared to a pure ADHD without comorbidity group. The results in the present dissertation showed that certain cognitive impairments, notably verbal working memory, verbal memory, concept shifting, and sustained attention processes are specific for adult ADHD and not so much accounted for by the presence of comorbidity (Chapter 2, 3, and 4). In addition to these findings, we also found that the ADHD adults with comorbidity showed a specific pattern of cognitive weakness (e.g., focused attention processes) that did not appear in the pure ADHD adults or in the non-ADHD pathological group (Chapter 3). From the present results it can be concluded that it is important to take note of comorbidity in the assessment of ADHD. For one, this clearly shows that specific cognitive deficits in which working memory plays an important role are found in ADHD adults. Secondly, these results indicate that these comorbid disorders, such as depressive or anxious complaints, low self esteem and usually present before young adulthood may additionally severe their symptoms and account for other cognitive deficits. Accordingly, there is considerable support for the notion that tasks requiring higher information processing demands and effort can be used to detect specific differences between ADHD, regardless of comorbidity, and general non-ADHD pathological control adults.

Secondly, the investigation of specificity of findings of impairments in EF and cognitive processes can also be useful in detecting differences in the cognitive profile in ADHD subtypes in adults. In the present dissertation, one study was included that examined the cognitive processes in adult ADHD subtypes (combined versus inattentive type) (Chapter 4). Data on the performance measures showed that the adults with the ADHD combined subtype had deficits in verbal working memory, verbal learning, retention and delayed recall measures compared with healthy controls, but not in comparison with the inattentive subtype adults. No significant differences were found in the use of organisational strategies. However, a closer inspection of the data revealed that while both subtypes favor a more simple and passive kind of organisational strategy, the ADHD combined subtype adults are less capable in adopting a more complex and active 
response organisation strategy than the inattentive subtype adults. Clearly, this suggests that both adult ADHD subtypes differ in their efficiency in using underlying response strategies. In turn, this inefficient strategy use may affect the verbal memory processes in the adult ADHD combined subtype as they can adopt fewer executive compensation strategies due to deficits in inhibition and verbal working memory. This study was the first to reveal such findings regarding different aspects of verbal memory and underlying cognitive processes and adult ADHD subtypes. Likewise, the results of this study provided some support for a dimensional view of ADHD (R. A. Barkley, 1998; Levy, Hay, McStephen, Wood, \& Waldman, 1997), in that pure ADHD in ADHD adults cannot be considered as one entity, but need to be subdivided into subtypes. Moreover, the results from this study showed that there was a linear effect on most of the verbal memory measures with ADHD adults with the combined type performing worse at one end, the healthy control adults performing best at the other end and the ADHD inattentive type adults performing somewhere in between. Evidently, the behavioral symptoms of adult ADHD do not lead to the same poor performan-ce on cognitive tasks in all ADHD subtypes. This suggests that, in adulthood, both ADHD subtypes can be regarded as two different entities or disorders with different deficits and mechanisms that are involved (Castellanos \& Tannock, 2002; Diamond, 2005; Gansler et al., 1998). This is an important finding in the present dissertation as research on the differences in verbal memory profile between ADHD subtypes is lacking in the adult population. Clearly, we can say that adults with both attentional and hyperactive and impulsive symptoms, have difficulty in daily life on holding their new incoming information present for a short time, and thereby resulting in they easily forget what they had planned or intended to do (e.g., what to buy in the supermarket or at work), not knowing how to prioritize their goals, and learning or memorizing things that are verbally presented to them. As a consequence, these adults experience at lot of stress and failures in different settings (e.g., at home, work that may further induce the cooccurrence psychiatric problems.

\section{Future research and clinical implications}

\section{Implications for future research}

The findings of the present dissertation have implications for future research and its theoretical context in order to better understand the cognitive mechanisms that are involved in adult ADHD. A first point relates to the relevance of new process approaches and analyses next to standard test performance. Our process analysis appeared to be very valuable and sensitive in determining the cognitive processes underlying the overall performance deficits in adults with ADHD. The Chapters 3, 4, 5 and 6 in this dissertation have shown that traditional overall outcome scores only provide a fraction of information on the core cogni- 
tive deficits in adults with ADHD. Therefore, we suggest that the various existing paper and pencil tasks and computerised tests of EF, should be re-evaluated in terms of the underlying cognitive processes. Also, new experimental or clinical tests should take this process approach into consideration.

Secondly, improve-ments in the theoretical considerations of adult ADHD can be made by further evaluating the proposed broadened model of EF by Peter Anderson (2002). Investigation of both cognitive and more motivational processes would shed more light on the proposed dual pathway model in adults with ADHD. In the present dissertation, this aspect was addressed by examining the cognitive deficits and some underlying cognitive processes that related to a number of motivational processes (e.g., 'state factors'). More research is needed on this model in ADHD adults that also integrates other motivational processes (e.g., delay aversion), but also more affective components of cognition (e.g., risk-taking, judgment).

Thirdly, the results of the studies described in this dissertation suggest that future research should take the influence of comorbidity and the distinctiveness of adult ADHD subtypes into account. In particular, our findings show that taking these issues into account can help us in drawing conclusions about whether the cognitive weaknesses and strengths in ADHD adults are specific for this disorder and for which kind of ADHD subtype. Furthermore, by including more ADHD groups with distinct comorbid disorders (e.g., mood disorder group and/or anxiety group), but also ADHD adults with subclinical levels of ADHD symptoms ('borderline' ADHD) and subdivided into ADHD subtypes, would add to our knowledge on the dimensional view of adult ADHD.

\section{Implications for clinical practice}

The present findings of the studies described in this dissertation have several implications for clinical practice. Firstly, we report on the first data for a Dutch population with adult ADHD that can be applied as a source for neuropsychological assessment of adult ADHD in clinical practice. We suggest that it is important to use a broad test battery, incorporating both traditional cognitive tasks and more information processing reaction time tasks to detect the cognitive strengths and weaknesses in adults with ADHD and differentiate them from other usually comorbid psychopathology. As shown from the studies described in this dissertation, the ADHD adults showed deficits on traditional paper and pencil tests (Chapter 2, 4, 5), but also on these more complex computerised reaction time tasks (Chapter 3 and 6). Likewise, clinicians should also focus more on the cognitive processes underlying the overall test performance as shown from the findings of this dissertation. In addition, by observing these ADHD adults in a more qualitative way would contribute to how they reach their standard outcome scores. Thus, there is great need for numerous cognitive tasks to be re-evaluated for clinical use based on this process analysis. 
Furthermore, the studies reported in the present dissertation stress out how stimulant medication methylphenidate (MPH) can be used as a tool for examining the complex cognitive processes in adults with ADHD (Chapter 5 and 6). Our findings showed that ADHD adults with the combined type had more difficulties in automatic processing of specifically semantically-related information while taking MPH medication (Chapter 5). Also, use of MPH medication in these ADHD combined adults significantly improved accuracy (i.e., impulsive responding by choice error rate), but not so much their attention and inhibition processes (Chapter 6). Therefore, future research on efficacy of stimulant medication using more controlled clinical designs should focus more on the underlying cognitive processes instead of solely the standard test performance. They should also include larger samples of ADHD adults while on and off stimulant medication. Based from our studies reported in Chapter 5 and 6, it is also recommended to perform follow-ups starting off after one month when starting stimulant pharmacotherapy to detect potential residual cognitive deficiencies that can still have impact on the daily functioning in the ADHD adults.

Thirdly, when considering the role of the neuropsychologist in the diagnostic process of adult ADHD some comments can be made. For one, there is no single cognitive test or test battery that can be used for the $100 \%$ diagnosis of adult ADHD. Instead, based from studies described in the present dissertation, a thorough interview with the patient and an informant are important to screen for adult ADHD. This interview should include a history of the ADHD complaints in the past childhood and present adulthood testing the persistence of the disorder, but also more cognitive complaints, questionnaires and the consideration of comorbidity. As ADHD is also considered to be a neuropsycological and not only a psychiatric disorder as precluded in the DSM classification system, we believe that multidisciplinary teams should be established as has been the case in the studies reported here. In these teams, an important role should be given to the neuropsychologist in addition to the psychiatrists and specialized health care psychologist. In addition, the neuropsychologist can contribute in an important way to establish and perform neuropsychological interventions in ADHD adults.

In line with this, we also contend that the findings from this dissertation have important implications for treatment interventions in adults with ADHD. As shown in this dissertation, ADHD adults, in particular those with the combined subtype have more problems in their working memory and learning of verbal unstructured information. They also had problems in how to efficiently use memory strategies to cope with learning problems. Therefore, we believe that adults with the combined type could benefit from working memory training in which they get more insight into these processes of learning, how these deficits influence their daily life and learn more to use alternative strategies to cope with these deficiencies. This working memory training has been found to effective in children with ADHD (Klingberg et al., 2005), but needs to be further investigated in adults with ADHD. In addition, given the high variability that we found in the ADHD 
adults indicative of more attentional problems, we contend that these working memory training are combined with attention regulation training and psychoeducation on what this disorder is and how these cognitive deficits affect their daily life. Attention and working memory processes have been closely linked together in such a way that tasks that involve working memory also involve a certain amount of attention and that this aspect is also addressed in the training. In addition, from clinical experience it is known that getting the diagnosis of ADHD can raise some unknown or never experienced emotions in these adults. Therefore, we recommend that neuropsychological interventions also target this emotional aspect of coping with the disorder.

\section{Conclusion}

ADHD is serious and prevalent disorder from childhood to adulthood that has a large impact on the adult individual. Yet, little cognitive research has been conducted on these adults diagnosed with ADHD in adulthood. It is an important field to study because of the important implications that research can have on clinical practice, such as the understanding of this disorder through development for clinicians. Also, this cognitive research can contribute to detecting the cognitive deficits and underlying processes and thus relieving the ADHD adults from their lifelong label of 'being lazy or stupid' and the under-diagnosis they are often have to cope with, when justification for their deficits can be evidenced scientifically. The results in the present dissertation showed that ADHD adults have certain specific weaknesses in more complex cognitive processes. In the future, research and clinicians should focus therefore more on the cognitive processes underlying overall test performance. In addition, the present dissertation is the first that stresses the important value of comorbidity in cognitive research in adults with ADHD. Moreover, future research should take the influence of comorbidity and the distinctiveness of adult ADHD subtypes into account as this can provide valuable information on the specificity of the cognitive deficits in ADHD adults. Finally, the need for development of neuropsychological interventions that focus on a combination of psycho-education, cognitive skills training (e.g., working memory, attention) and emotional components to improve the well-being and quality of life of the adult individuals suffering from ADHD is stressed. 


\section{References}

Alvarez, J. A., \& Emory, E. (2006). Executive function and the frontal lobes: A meta-analytic review. Neuropsychology Review, 16(1), 17-42.

Anderson, P. (2002). Assessment and development of executive function (EF) during childhood. Child Neuropsychology, 8(2), 71-82.

Barkley, R. A. (1997). Behavioral inhibitions, sustained attention, and executive functions: constructing a unifying theory of ADHD. Psychological Bulletin, 121, 65-94.

Barkley, R. A. (1998). Attention-Deficit/ Hyperactivity Disorder: A handbook for diagnosis and treatment (2nd ed.). New York: The Guilford Press.

Barkley, R. A., Murphy, K., \& Kwasnik, D. (1996). Psychological adjustment and adaptive impairments in young adults with ADHD. Journal of Attention Disorders, 1 (1), 41-54.

Börger, N., Van der Meere, J., Ronner, A., Alberts, E., Geuze, R., \& Bogte, H. (1999). Heart rate variability and sustained attention in ADHD children. Journal of Abnormal Child Psychology, 27(1), 25-33.

Castellanos, F. X., \& Tannock, R. (2002). Neuroscience of attention-deficit/hyperactivity disorder: the search for endophenotypes. Nature Reviews Neuroscience, 3, 617-628.

Diamond, A. (2005). Attention-deficit disorder (attention-deficit/hyperactivity disorder without hyperactivity): A neurobiologically and behaviorally distinct disorder from attention-deficit/hyperactivity disorder (with hyperactivity). Development and Psychopathology, 17, 807-825.

Faraone, S. V., Biederman, J., \& Mick, E. (2005). The age-dependent decline of attention deficit hyperactivity disorder: A meta-analysis of follow-up studies. Psychological Medicine, 36, 159-165.

Gansler, D. A., Fucetola, R., Krengel, M., Stetson, S., Zimering, R., \& Makary, C. (1998). Are there cognitive subtypes in adult Attention Deficit/Hyperactivity Disorder? The Journal of Nervous and Mental Disease, 186, 776-781.

Hervey, A. S., Epstein, J. N., \& Curry, J. F. (2004). Neuropsychology of adults with AttentionDeficit/Hyperactivity Disorder: A meta-analytic review. Neuropsychology, 18(3), 485503.

Houx, P. J., \& Jolles, J. (1993). Age-related decline of psychomotor speed: effects of age, brain health, sex, and education. Perceptual and Motor Skills, 76(1), 195-211.

Houx, P. J., Vreeling, F. W., \& Jolles, J. (1991). Age associated cognitive decline is related to biological life-events. In R. C. Mclachlan, B. Winblad \& H. M. Wisniewski (Eds.), Alzheimer's Disease: Basic mechanisms, diagnosis and therapeutic strategies. (pp. 353-359). Chichester: Wiley.

Hurks, P. P. M., Hendriksen, J. G. M., Vles, J. S. H., Kalff, A. C., Feron, F. J. M., Kroes, M., et al. (2004). Verbal fluency over time as a measure of automatic and controlled processing in children with ADHD. Brain and Cognition, 55, 535-544.

Kalff, A. C. (2002). Neurocognitive performance and demographic variables in children at risk of Attention-Deficit/Hyperactivity Disorder. Maastricht: Neuropsych Publishers.

Kalff, A. C., De Sonnevile, L. M. J., Hurks, P. P. M., Steyaert, J., Zeben, T. M. C. B. v., Vles, J. S. H., et al. (2003). Low- and high-level controlled processing in executive motor control tasks in 5-6-year-old children at risk of ADHD. Journal of Child Psychology and Psychiatry, 44 (7), 1049-1057. 
Klingberg, T., Fernell, E., Olesen, P. J., Johnson, M., Gustafsson, P., Dahlström, K., et al. (2005). Computerized training of working memory in children with ADHD- A randomized, controlled trial. Journal of the American Academy Child and Adolescence Psychiatry, $44(2), 177-186$.

Kuntsi, J., Oosterlaan, J., \& Stevenson, J. (2001). Psychological mechanisms in hyperactivity: I Response inhibition deficit, working memory impairment, delay aversion, or something else? Journal of Child Psychology and Psychiatry, 42 (2), 199-210.

Levy, F., Hay, D. A., McStephen, M., Wood, C., \& Waldman, I. (1997). Attention-Deficit Hyperactivity Disorder: A category or continuum? Genetic analysis of a large-scale twin study. Journal of the American Academy of Child and Adolescent Psychiatry, 36, 737-744.

Lezak, M. D. (1995). Neuropsychological assessment (3rd ed.). Oxford: Oxford University Press.

Marks, D. J., Newcorn, J. H., \& Halperin, J. M. (2001). Comorbidity in Adults with AttentionDeficit/Hyperactivity Disorder. Annals of New York Academy of Sciences, 931, 216238.

Milberger, S., Biederman, J., Faraone, S. V., Murphy, J., \& Tsuang, M. T. (1995). Attention deficit hyperactivity disorder and comorbid disorders: issues of overlapping symptoms. American Journal of Psychiatry, 152, 1793-1799.

Millstein, R. B., Wilens, T. E., Biederman, J., \& Spencer, T. J. (1997). Presenting ADHD symptoms and subtypes in clinically referred adults with ADHD. Journal of Attention Disorders, 2(3), 159-166.

Pennington, B. F. (2005). Toward a new neuropsychological model of attention-deficit/ hyperactivity disorder: Subtypes and multiple deficits. Biological Psychiatry, 57, 12211223.

Pennington, B. F., \& Ozonoff, S. (1996). Executive functions and developmental psychopathology. Journal of Child Psychology and Psychiatry, 37, 51-87.

Rucklidge, J. J., \& Tannock, R. (2002). Neuropsychological profiles of adolescents with ADHD: effects of reading difficulties and gender. Journal of Child Psychology and Psychiatry, 43(8), 988-1003.

Seidman, L. J. (2006). Neuropsychological functioning in people with ADHD across the lifespan. Clinical Psychology Review, 26, 466-485.

Salthouse, T. A. (1996). The processing-speed theory of adult age differences in cognition. Psychological Review, $103(3), 403-428$.

Sergeant, J. A. (2005). Modeling Attention-Deficit/Hyperactivity Disorder: A critical appraisal of the cognitive-energetical model. Biological Psychiatry, 57, 1248-1255.

Sergeant, J. A., Geurts, H., \& Oosterlaan, J. (2002). How specific is a deficit of executive functioning for Attention-Deficit/Hyperactivity Disorder? Behavioral Brain Research, 130, 3-28.

Sonuga-Barke, E. J. S. (2005). Causal models of Attention-Deficit/Hyperactivity Disorder: From common simple deficits to multiple developmental pathways. Biological Psychiatry, 57, 1231-1238.

Spreen, O., \& Strauss, E. (1998). A compendium of neuropsychological tests: Administration, norms, and commentary. (2nd ed.). New York: Oxford University Press. 
Willcutt, E. G., Doyle, A. E., Nigg, J. T., Faraone, S. V., \& Pennington, B. F. (2005). Validity of the executive function theory of Attention-Deficit/Hyperactivity Disorder: A metaanalytic review. Biological Psychiatry, 57, 1336-1346.

Woods, S. P., Lovejoy, D. W., \& Ball, J. D. (2002). Neuropsychological characteristics of adults with ADHD: A comprehensive review of initial studies. The Clinical Neuropsychologist, $16(1), 12-34$. 



\section{Summary}


154 | Summary 
Attention Deficit Hyperactivity Disorder (ADHD) is a highly prevalent neurodevelopmental syndrome that persists into adulthood. We now know from the many childhood studies that this syndrome is characterized by multiple cognitive deficits, such as problems on the domain of executive functioning. However, far less research has been conducted on adults with ADHD. Also, the cognitive processes underlying decreased performance on standard summary scores in adults with ADHD have, to date, been inadequately addressed and poorly understood. Therefore, the central theme of this dissertation was to disentangle the more complex cognitive processes, notably executive functions, underlying test performance in adults with ADHD. The complex cognitive functions described in the present dissertation were chosen based on research findings in children with ADHD and for their importance in daily life of adults with ADHD as reported in clinical practice. In order to achieve this, a number of issues were addressed, including (i) the impact of comorbidity and, thus, specificity of the cognitive deficits in adults with ADHD; (ii) whether ADHD subtypes can be differentiated from each other by use of alternative measures for cognitive processes underlying their learning performance; and (iii) whether methylphenidate can be used as a tool to further explore these underlying cognitive processes in adult ADHD. In light of these aims, several cross-sectional studies were conducted. Each of these studies focused on one or more distinct cognitive functions in adults diagnosed with ADHD (combined or inattentive subtype). The age range in the studies varied from 18 to 61 years, because of the known age-related cognitive deterioration after 60 years and the influence of (pre-) retirement in adults over the age of 61 (Houx, \& Jolles, 1993; Houx, Vreeling, \& Jolles 1991; Salthouse, 1996).

Chapter 1 gives a general introduction into adult ADHD and explains the rationale for the central theme of this dissertation. Additionally, the term 'executive functions' (EF) is described based on the developmental model of executive function proposed by Peter Anderson (2002). This EF model was used as the building block to incorporate the cognitive functions and higher-order processes that were the object of the studies reported in the present dissertation. Following the description of this model, a more detailed overview and reasons for the use of the new process approaches that were applied in a number of studies described in this dissertation is given. Next, a discussion of three important issues when studying cognitive functioning in adult ADHD is provided. This chapter ends with stating the central theme and aims targetted in the present dissertation and conveys a short overview of the following chapters.

Chapter 2 describes a study that investigated the specificity of four domains of executive functioning in adults with ADHD. These domains included interference control, concept shifting, verbal fluency, and verbal working memory. This was examined by comparing four groups of participants, including (i) 20 adults diagnosed with ADHD (ADHD), (ii) 22 adults diagnosed with both ADHD 
and one or more comorbid disorder(s) $\left(\mathrm{ADHD}^{+}\right.$), (iii) 34 adults referred for $A D H D$ due to ADHD symptomatology but not diagnosed as such (non-ADHD) and (iv) 136 healthy controls matched for gender, age and $I Q$, on tasks measuring various executive functions. The results showed that ADHD- and ADHD+ groups exhibited specific deficits on concept shifting and verbal working memory compared to the control groups. This provided rather evidence for ADHD-related deficits, regardless of comorbidity, on these two domains of executive functioning. In addition, the $\mathrm{ADHD}^{+}$and non-ADHD groups displayed deficits in terms of general simple processing speed. Given that these deficits were not found in the ADHD- group, we concluded that these processing deficits are likely attributable to comorbidity rather than ADHD itself. Contrary to our expectations, the finding of additional cognitive deficits in the ADHD with comorbidity adults did not correspond with the cognitive subtype hypothesis (Rucklidge \& Tannock, 2002). Instead, we tentatively suggested that the effects due to the presence of ADHD and one or more comorbid disorders result from the additive combination of the correlates of each disorder when they occur separately as suggested by (Willcutt, Pennington, Olson, Chhabildas, \& Hulslander, 2005).

Chapter 3 reports a study that examined the issues of the nature and specificity of the deficits in focused attention and sustained attention in adults with Attention Deficit Hyperactivity Disorder (ADHD). In this study, the process approach was applied by investigating the time-on task and intra-trial variability as possible underlying cognitive processes in adult ADHD. Twenty-eight adults with ADHD without comorbidity (ADHD) were compared with 28 ADHD outpatients with comorbidity $\left(\mathrm{ADHD}^{+}\right), 68$ adults referred for $\mathrm{ADHD}$ but with another psychopathology than ADHD (non-ADHD) and 28 healthy controls, matched for gender, age and IQ, on attention tests of the Amsterdam Neuropsychological Tasks program. Both ADHD groups, irrespective of comorbidity, demonstrated a sustained attention deficit relative to the control groups, as indicated by a disproportionate deterioration of speed fluctuation with time-on-task reflecting temporal lapses in attention. Only the $\mathrm{ADHD}^{+}$group showed focused attention deficits in that they were less able to ignore irrelevant information by a lower accuracy compared with all three comparison groups. These findings indicated that adults with ADHD have specific deficits in sustained attention. Additional deficits in focused attention were viewed to be rather confined to outpatients with ADHD and comorbidity. Our findings regarding deficient attentional processes in adult with ADHD rather suggest that differential underlying processes and correlates may be involved in ADHD adults with and without comorbidity. Also, these results provide strong support for using more process approaches in adults with ADHD in distinguishing not only adults with ADHD from other psychopathologies, but also in detecting differences in attention processes between ADHD with and without comorbidity. 
Chapter 4 presents the findings of a study on verbal learning and organisational strategies and the role of comorbidity in two subtypes of adult ADHD. Ninety-eight adults with ADHD combined type (ADHD-C), 37 adults with ADHD inattentive type (ADHD-IA), and 119 gender- and age matched healthy controls were compared while performing an auditory verbal learning task. Comorbidity was included in both ADHD groups and was controlled for by including it as a nominal variable (comorbidity versus no comorbidity) in all analyses as a betweensubject factor in order to determine whether there was an interaction between the subgroups and psychiatric comorbidity. The results showed that, regardless of comorbidity, the ADHD combined group exhibited deficits in verbal working memory, learning, retention, and delayed recall relative to the healthy control subjects. These deficits did not emerge for the ADHD inattentive group. No statistically significant group differences in serial and subjective organisational strategies emerged. However, the hierarchical linear regression analyses showed that use of such strategies predicted verbal learning performance. There was no effect or interaction found for comorbidity in all analyses. Moreover, the linear effects showed that the ADHD-IA group performed better than the ADHD-C group but poorer than the controls. These findings provided support for the hypothesis that ADHD is a continuum involving distinct subtypes (Barkley, 1998; Levy, Hay, McStephen, Wood, \& Waldman, 1997) which can be identified by verbal memory impairments. It was further suggested that a deficient inhibition plays a role in memory impairments in the ADHD-C subtype as these could not be found in the ADHD-IA subtype.

Chapter 5 reports the results of a comparison of automatic and controlled strategies on semantic category fluency (SCF) and initial letter fluency (ILF) in adults ADHD and healthy control subjects. In addition to the traditional overall scores on verbal fluency tasks, word production for each 15-s time slice, and word clustering, switching and errors over $60 \mathrm{~s}$ was calculated for both fluency tasks. The reason for this process approach was that it provides more insight into automatic and controlled cognitive processing or underlying process in adults with ADHD. The present study was the first to address this topic in medicated adults with ADHD. To this end, 16 adults with ADHD, combined type, were compared to 15 age- and education-matched healthy controls. Thirteen ADHD adults were taking methylphenidate $(\mathrm{MPH})$ medication; the remainder three adults were treatment naïve. The most salient finding was that the ADHD adults generated a significantly lower number of words during the first $15 \mathrm{~s}$ as compared to healthy controls on the SCF. This was not found to be the case on the ILF, in contrast the medicated ADHD adults showed better performance on automatic processing on the ILF task. No group differences in controlled information processing, errors, clustering and switching strategies emerged on both types of fluency. These results remained the same when we repeated our analyses without the three medication naïve ADHD adults, indicating valid results. These fin- 
dings were placed in the context that adults with ADHD have difficulty with automatic information processing from the semantic memory store, despite MPH treatment, and that this more likely can be considered as a pervasive ADHDrelated deficit.

Chapter 6 describes a study with the aim to assess the efficacy of stimulant medication methylphenidate (MPH) on inhibition and attention processes in adults with ADHD. This was done to test the hypothesis whether a primary disorder of inhibitory control or a more general deficit in inattention is apparent in adult ADHD. Thirteen adults diagnosed with ADHD combined type, while taking MPH and while not taking MPH, were compared to 15 healthy participants, matched for age and educational and also assessed twice, on a stop-signal task. It was demonstrated that the ADHD adults off MPH medication had a slower stop-signal reaction time (SSRT), a larger variability in go response speed (SDRT), and made more choice errors than the healthy controls. Furthermore, choice error rate, but no other measures of inhibition and attention, was specifically improved in the ADHD adults when taking MPH medication compared to when not on MPH. In addition, a significant higher variability in response speed remained when ADHD adults where on and off MPH medication. These findings were interpreted as evidence that ADHD in adults is characterised by a more global deficit in attention regulation affecting inhibition of a response. In addition, the results confirmed that a MPH treatment primarily ameliorates impulsive responding in adults with ADHD.

Chapter 7 provides some concluding remarks on issues which are discussed in the context of the theme of the present dissertation and recent literature. Overall, the present dissertation is the first to provide more insight into the more complex cognitive processes underlying standard test performance in adults with ADHD. In the future, research and clinicians should focus therefore more on the underlying cognitive processes instead on using only the overall test scores. In addition, the present dissertation is the first that stresses the important value of comorbidity and ADHD subtypes in cognitive research in adults with ADHD. Moreover, future research should take the influence of comorbidity and the distinctiveness of adult ADHD subtypes into account as this can provide valuable information on the specificity of the cognitive deficits in ADHD adults. Lastly, based on the outcome of the studies outlined in this dissertation, various implications for future research and for clinical practice are provided. The need for development of neuropsychological interventions that focus on a combination of psycho-education, cognitive skills training (e.g., working memory, attention) and emotional components to improve the well-being and quality of life of the adult individuals suffering from ADHD is stressed. 


\section{References}

Anderson, P. (2002). Assessment and development of executive function (EF) during childhood. Child Neuropsychology, 8(2), 71-82.

Barkley, R. A. (1998). Attention-Deficit/ Hyperactivity Disorder: A handbook for diagnosis and treatment (2nd ed.). New York: The Guilford Press.

Houx, P. J., \& Jolles, J. (1993). Age-related decline of psychomotor speed: effects of age, brain health, sex, and education. Perceptual and Motor Skills, 76(1), 195-211.

Houx, P. J., Vreeling, F. W., \& Jolles, J. (1991). Age associated cognitive decline is related to biological life-events. In R. C. Mclachlan, B. Winblad \& H. M. Wisniewski (Eds.), Alzheimer's Disease: Basic mechanisms, diagnosis and therapeutic strategies. (pp. 353-359). Chichester: Wiley.

Levy, F., Hay, D. A., McStephen, M., Wood, C., \& Waldman, I. (1997). Attention-Deficit Hyperactivity Disorder: A category or continuum? Genetic analysis of a large-scale twin study. Journal of the American Academy of Child and Adolescent Psychiatry, 36, 737-744.

Rucklidge, J. J., \& Tannock, R. (2002). Neuropsychological profiles of adolescents with ADHD: effects of reading difficulties and gender. Journal of Child Psychology and Psychiatry, 43(8), 988-1003.

Salthouse, T. A. (1996). The processing-speed theory of adult age differences in cognition. Psychological Review, 103(3), 403-428.

Willcutt, E. G., Pennington, B. F., Olson, R. K., Chhabildas, N., \& Hulslander, J. (2005). Neuropsychological analyses of comorbidity between reading disability and attention deficit hyperactivity disorder: In search of the common deficit. Developmental Neuropsychology, 27(1), 35-78. 

Samenvatting 
162 | Samenvatting 
Attention Deficit Hyperactivity Disorder - in het Nederlands Aandachtstekortstoornis met Hyperactiviteit ('ADHD')- is een veelvoorkomende ontwikkelingsstoornis die in vele gevallen blijft bestaan tot in de volwassenheid. Uit de vele studies die zijn verricht bij kinderen is duidelijk geworden dat dit syndroom gekenmerkt wordt door verschillende cognitieve stoornissen. Centraal staan problemen ten aanzien van het executief functioneren ofwel de planning- en controlefuncties. Tot dusver is nog niet veel onderzoek dat zich heeft gericht op volwassenen met ADHD. Daarnaast is nog maar weinig bekend over de cognitieve processen die ten grondslag liggen aan de verminderde cognitieve prestaties bij volwassenen met ADHD. Het centrale thema van dit proefschrift wordt gevormd door deze complexe cognitieve processen, - in het bijzonder de executieve functies. De verschillende complexe cognitieve functies die zijn beschreven in het voorliggende proefschrift zijn gekozen op basis van de bevindingen bij kinderen met ADHD, maar ook omdat deze, op basis van rapportages vit de klinische praktijk, belangrijk zijn gebleken voor het dagelijks functioneren van volwassenen met ADHD. Om de algemene probleemstelling te beantwoorden komen de volgende vraagstellingen aan de orde, namelijk (i) wat is de impact van co-morbiditeit en meer bepaald de specificiteit van de cognitieve tekorten bij volwassenen met ADHD, (ii) kunnen ADHD subtypes van elkaar onderscheiden worden aan de hand van cognitieve processen onderliggend aan de leerprestatie; en (iii) kan Methylphenidaat (Ritalin) gebruikt worden als een hulpmiddel om deze onderliggende cognitieve processen verder te exploreren bij volwassenen met ADHD. Om deze doelstellingen te beantwoorden zijn verschillende cross-sectionele studies uitgevoerd. Elke studie richt zich op een of meerdere cognitieve functies bij volwassenen met ADHD. Daarbij gaat het om het gecombineerde en/of inattente subtype. De leeftijd in deze studies varieert van 18 tot 61 jaar. Deze leeftijdsgrens is gekozen omwille van het feit dat bekend is dat de leeftijdsgeassocieerde cognitieve achteruitgang vooral inzet na 60 jaar en vanwege de invloed van psychosociale factoren zoals het (pre-)pensioen bij senioren voorbij 61 jaar (Houx, \& Jolles, 1993; Houx, Vreeling, \& Jolles 1991; Salthouse, 1996).

Hoofdstuk 1 geeft een algemene inleiding over ADHD bij volwassenen en verklaart de keuze voor het centrale thema in dit proefschrift. Daarnaast wordt de term 'executieve functies' (EF) uitgelegd aan de hand van het ontwikkelingsmodel van executief functioneren zoals geformuleerd door Peter Anderson (2002). Dit EF model wordt gebruikt als basis, waarbij een link gemaakt wordt met de cognitieve functies en hogere orde processen die onderzocht worden in de studies beschreven in dit proefschrift. Volgend op de beschrijving van dit model wordt een beknopt overzicht gegeven van de nieuwe cognitief neuropsychologische methoden die worden toegepast in een aantal studies beschreven in dit proefschrift. Tevens worden de beweegredenen voor het gebruik van deze methoden viteengezet. Kort samengevat zijn deze beweegredenen de volgende: 1) meer inzicht in automatische en gecontroleerde informatieverwerkingspro- 
cessen en variabiliteit te krijgen, en 2) beter zicht op de executieve organisatie strategieën. Vervolgens worden drie belangrijke onderwerpen besproken die van belang zijn voor het bestuderen van het cognitief functioneren bij volwassenen met ADHD. Dit hoofdstuk eindigt met een beschrijving van het centrale thema en de doelstellingen van dit proefschrift en een beknopt overzicht van de hieropvolgende hoofdstukken.

Hoofdstuk 2 beschrijft de resultaten van een studie waarbij de specifici-teit op vier domeinen van executief functioneren onderzocht bij volwassenen met ADHD. Deze vier domeinen betreffen interferentie controle, concept shifting, verbale woordvlotheid (fluency), en verbaal werkgeheugen. Deze domeinen worden onderzocht door en vergelijking van vier groepen: (i) volwassenen gediagnosticeerd met enkel ADHD (ADHD', $n=20$ ), (ii) volwassenen gediagnosticeerd met zowel ADHD en één of meerdere co-morbide stoornis(sen) (ADHD+; $n$ 22), (iii) volwassenen, verwezen voor ADHD naar aanleiding van hun ADHDachtige symptomen, maar viteindelijk niet voldeden aan de criteria van ADHD (niet-ADHD; $n=34$ ), en (iv) gezonde controleproefpersonen ( $n=136$ ), gematcht op geslacht, leeftijd en IQ, op taken die verschillende executieve functies beogen te meten. Uit de analyses blijkt dat de ADHD- and ADHD+ groepen speciieke tekorten vertonen op concept shifting en verbaal werkgeheugen vergeleken met de controle groepen. Deze resultaten leveren bewijs voor tekorten gerelateerd aan ADHD stoornis en onafhankelijk van de co-morbiditeit, op deze twee domeinen van executief functioneren. Daarnaast vertonen de $\mathrm{ADHD}^{+}$en niet-ADHD groepen stoornissen op het gebied van algemene eenvoudige snelheid van informatieverwerking. Omdat deze stoornissen niet teruggevonden zijn bij de volwassenen in de ADHD- groep, kunnen we concluderen dat deze informatieverwerkingsstoornissen eerder toe te schrijven zijn aan de co-morbiditeit in plaats van de ADHD symptomen. In tegenstelling tot onze verwachting, kwam de bevinding van niet-specifieke bijkomende cognitieve stoornissen bij volwassenen met ADHD en co-morbiditeit niet overeen met de cognitieve subtype hypothese (Rucklidge \& Tannock, 2002). De suggestie wordt dan ook gedaan dat de effecten van ADHD en co-morbide stoornissen een optelsom zijn van de beperkingen behorende bij iedere stoornis apart. Zo wordt ook gedacht door Willcutt, Pennington, Olson, Chhabildas, en Hulslander (2005).

Hoofdstuk 3 beschrijft een onderzoek naar de aard en specificiteit van stoornissen in gerichte en volgehouden aandachtsprocessen bij volwassenen met ADHD. In deze studie wordt uitgegaan van een proces benadering. Daarbij wordt de tijd onderzocht die de vitvoering van een taak kost, evenals de binnentaak variabiliteit. Hierbij worden de prestaties van 28 volwassenen met ADHD en zonder co-morbiditeit (ADHD') vergeleken met 28 ADHD patiënten met co-morbiditeit $\left(\mathrm{ADHD}^{+}\right), 68$ volwassenen verwezen voor $\mathrm{ADHD}$ naar aanleiding van hun ADHD-achtige symptomen, maar viteindelijk niet voldeden aan de criteria van 
ADHD (niet-ADHD), en 28 gezonde proefpersonen die zijn gematcht op geslacht, leeftijd en IQ. De prestatie wordt gemeten op gecomputeriseerde aandachtsta-ken van het Amsterdamse Neuropsychologische Taken (ANT) programma. Uit de resultaten blijkt dat beide ADHD groepen, ongeacht het wel of niet hebben van co-morbiditeit, zich kenmerkten door een stoornis in de volgehouden aandacht. Deze stoornis blijkt vit een disproportioneel grotere variabiliteit in de snelheid van reageren over de tijd heen op een volgehouden aandachtstaak bij volwassenen met ADHD vergeleken met de overige controle groepen. Dit weerspiegelt ook wel een tijdelijk verval in hun aandacht. Daarnaast heeft enkel de $\mathrm{ADHD}^{+}$groep specifieke stoornissen in de gerichte aandacht. De ADHD volwassenen in deze groep zijn minder goed in staat om irrelevante informatie te onderdrukken. Dit resulteerde in een lagere accuratesse vergeleken met de drie andere controle groepen. Deze resultaten tonen aan dat volwassenen met ADHD specifieke vol-gehouden aandachtsstoornissen hebben. Daarnaast is aangetoond dat stoor-nissen in de gerichte aandacht vooral voorkomen bij volwassenen met ADHD en co-morbiditeit. Onze bevindingen met betrekking tot tekorten in aandachtspro-cessen suggereren dat verschillende onderliggende processen en correlaten betrokken zijn bij volwassenen met ADHD met en zonder co-morbiditeit. Tevens laten deze resultaten zien dat een proces aanpak kan bijdragen in het opsporen van verschillen in aandachtsprocessen bij volwassenen met ADHD die wel en geen co-morbiditeit hebben: dit pleit voor meer gebruik van een proces aanpak om volwassenen met ADHD te kunnen onderscheiden van andere vormen van psychopathologie.

Hoofdstuk 4 presenteert de resultaten van een studie naar verbaal leren en organisatie strategieën en de rol van co-morbiditeit bij twee subtypen van ADHD in de volwassenheid. Hiervoor zijn 98 volwassenen met ADHD met het gecombineerde subtype (ADHD-C), 37 volwassenen met ADHD predominante inattente subtype en 119 gezonde proefpersonen, gematcht op geslacht en leeftijd, vergeleken op een verbale woord leer taak. Het hebben van co-morbide stoornis(sen) is toegestaan in beide ADHD groepen en hiervoor wordt gecontroleerd door middel van co-morbiditeit als een nominale variabele (wel versus geen co-morbiditeit) toe te voegen in alle analyses. Dit is gedaan om te bepalen of er sprake is van een interactie tussen de groepsvariabele en psychiatrische co-morbiditeit. De resultaten geven aan dat de ADHD gecombineerde subtype groep gekenmerkt wordt door stoornissen op het vlak van verbaal werkgeheugen, leren, retentie en uitgestelde herinnering vergeleken met gezonde proefpersonen. Dit geldt, ongeacht of ze wel of geen vorm van psychiatrische co-morbiditeit hebben. Deze bevinding komt niet naar voren bij de ADHD inattente subtype groep. Verder zijn er geen statistisch significante groepsverschillen gevonden wat betreft seriële en subjectieve organisatie strategieën. In de hiërarchische lineaire regressie analyses komt echter naar voren dat het 
gebruik van deze strategieën voorspellers zijn voor verbale leerprestatie. Het hebben van co-morbiditeit heeft geen invloed op de resultaten. Daarnaast geven de lineaire effecten aan dat de ADHD inattente subtype groep doorgaans beter presteert dan de ADHD gecombineerde subtype groep maar slechter dan de gezonde proefpersonen. Deze bevindingen ondersteunen de hypothese dat ADHD een continuüm is bestaande vit verschillende subtypes (Barkley, 1998; Levy, Hay, McStephen, Wood, \& Waldman, 1997) die onderscheiden kunnen worden aan de hand van verbale geheugenbeperkingen. Tot slot wordt geopperd dat een stoornis in inhibitie een rol speelt bij geheugenbeperkingen bij het ADHD-C subtype aangezien deze niet terug gevonden worden bij het ADHDIA subtype.

Hoofdstuk 5 beschrijft de resultaten van een onderzoek naar de vergelijking van automatische en gecontroleerde strategieën bij semantische categorie fluency (SCF) en initiële letter fluency (ILF) bij volwassenen met ADHD en gezonde proefpersonen. Ten behoeve van dit onderzoek worden, naast de traditionele totaalscore, de volgende scores voor beide verbale woordvlotheid taken berekend: woordproductie per 15s-tijdsinterval, en woord clustering, switching en fouten over $60 \mathrm{~s}$. Deze proces benadering is gekozen om meer inzicht te verwerven over de onderliggende automatische en gecontroleerde cognitieve verwerking bij volwassenen met ADHD. Deze studie is ook de eerste die zich richt op dit onderwerp bij volwassenen met ADHD die medicatie gebruiken. Hiervoor zijn 16 volwassenen met het ADHD gecombineerde subtype vergeleken met 15 gezonde proefpersonen, gematcht op leeftijd en opleidingsniveau. Hierbij nemen 13 ADHD volwassenen Methylphenidaat (MPH) medicatie en de overige 3 subjecten hebben nog nooit een medicamenteuze behandeling gehad. De belangrijkste bevinding is dat de volwassenen met ADHD significant minder woorden opnoemen tijdens de eerste 15s op de SCF taak vergeleken met gezonde proefpersonen. Dit resultaat is niet terug gevonden op de ILF taak. In tegenstelling daarmee presteren de ADHD volwassenen met medicatie beter op automatische informatieverwerking (eerste $15 \mathrm{~s}$ ) op de ILF taak. Er zijn geen groepsverschillen gevonden wat betreft gecontroleerde informatieverwerking, fouten, clustering en switching strategieën op beide fluency taken. Er zijn geen verschillen naar voren gekomen wanneer de analyses herhaald werden zonder de drie medicatie-naïeve ADHD volwassenen, wat wijst op valide resultaten. Deze bevindingen worden geplaatst in de context ADHD-gerelateerde problemen met automatische informatieverwerking vanuit de opslag in het semantische geheugen. Daarenboven wordt deze beperking beschouwd als een pervasieve (onafhankelijk van medicatie bestaande) stoornis gerelateerd aan ADHD.

Hoofdstuk 6 presenteert een studie die gericht is op het meten van de effectiviteit van stimulerende medicatie Methylphenidaat (MPH) op inhibitie- en 
aandachtsprocessen bij volwassenen met ADHD. De reden om dit te onderzoeken is om de hypothese te toetsen of ADHD bij volwassenen gekenmerkt wordt door een primaire stoornis in inhibitoire controle of eerder door een algemeen tekort in de aandacht. Hiervoor zijn 13 volwassenen met het ADHD gecombineerd type vergeleken met 15 gezonde proefpersonen, die gematcht zijn op leeftijd en opleidingsniveau, ten aanzien van prestaties op een stop signaal inhibitie taak. De ADHD volwassenen zijn op twee meetmomenten getest, namelijk wanneer ze wel medicatie ingenomen hebben en wanneer ze medicatie vrij zijn en de gezonde proefpersonen zijn ook op twee meetmomenten getest (de tussentijd is vergelijkbaar voor de controles en de ADHD-ers). De resultaten laten zien dat de ADHD volwassenen zonder medicatie een tragere reactiesnelheid in het stoppen (SSRT) en een grotere variabiliteit bij het reageren vertonen en dat ze meer keuze fouten maken dan de controles. Daarnaast kan met deze studie worden aangetoond dat wanneer ADHD volwassenen wel MPH medicatie nemen er specifiek minder keuzefouten worden gemaakt vergeleken dan wanneer ze geen MPH medicatie nemen. Verder blijft er een significant hogere variabiliteit in snelheid van reageren aanwezig ongeacht of de ADHD volwassenen wel of geen MPH medicatie nemen. Deze bevindingen leveren bewijs voor de hypothese dat ADHD bij volwassenen gekarakteriseerd wordt door een globaal tekort in aandachtsregulatie dat op haar beurt de inhibitie van een respons beïnvloedt. Tenslotte bevestigen deze resultaten dat MPH behandeling met name een verbetering in impulsief gedrag bij volwassenen met ADHD oplevert.

Hoofdstuk 7 bespreekt de belangrijkste bevindingen en conclusies uit dit proefschrift in de context van het centrale thema en de bestaande en recent verschenen literatuur. Allereerst is het onderzoek dat in voorliggend proefschrift beschreven staat het eerste dat meer inzicht biedt in de complexe cognitieve processen die ten grondslag liggen aan de standaard test prestatie bij volwassenen met ADHD. De resultaten beschreven in dit proefschrift benadrukken dan ook het belang om naast de testscores die standaard in de praktijk worden gebruikt deze onderliggende cognitieve processen mee te nemen. Dit geldt zowel in het wetenschapsveld als in de klinische praktijk. Daarnaast wordt in dit proefschrift voor het eerst de belangrijke rol onderstreept van co-morbiditeit enerzijds en ADHD subtypes anderzijds in neurocognitief experimenteel onderzoek bij volwassenen met ADHD. In de toekomst zal het onderzoek daarom dienen rekening te houden met de invloed van co-morbiditeit en de te onderscheiden ADHD subtypes gezien deze belangrijke informatie opleveren over de specificiteit van cognitieve stoornissen bij ADHD volwassenen. Tot slot hebben de bevindingen uit de experimentele studies beschreven in dit proefschrift verschillende implicaties voor toekomstig onderzoek en voor de klinische praktijk. Deze worden in dit hoofdstuk nader toegelicht. Dit hoofdstuk sluit af met het advies voor meer neuropsychologische interventies gericht op drie belangrijke componenten: psycho-educatie, cognitieve vaardigheidstraining (bv. werkgeheugen, 
168 | Samenvatting

aandacht) en de emotionele aspecten, met als doel de verbetering van het welbevinden en kwaliteit van leven bij volwassenen met ADHD. 


\section{Referenties}

Anderson, P. (2002). Assessment and development of executive function (EF) during childhood. Child Neuropsychology, 8(2), 71-82.

Barkley, R. A. (1998). Attention-Deficit/ Hyperactivity Disorder: A handbook for diagnosis and treatment (2nd ed.). New York: The Guilford Press.

Houx, P. J., \& Jolles, J. (1993). Age-related decline of psychomotor speed: effects of age, brain health, sex, and education. Perceptual and Motor Skills, 76(1), 195-211.

Houx, P. J., Vreeling, F. W., \& Jolles, J. (1991). Age associated cognitive decline is related to biological life-events. In R. C. Mclachlan, B. Winblad \& H. M. Wisniewski (Eds.), Alzheimer's Disease: Basic mechanisms, diagnosis and therapeutic strategies. (pp. 353-359). Chichester: Wiley.

Levy, F., Hay, D. A., McStephen, M., Wood, C., \& Waldman, I. (1997). Attention-Deficit Hyperactivity Disorder: A category or continuum? Genetic analysis of a large-scale twin study. Journal of the American Academy of Child and Adolescent Psychiatry, 36, 737-744.

Rucklidge, J. J., \& Tannock, R. (2002). Neuropsychological profiles of adolescents with ADHD: effects of reading difficulties and gender. Journal of Child Psychology and Psychiatry, 43(8), 988-1003.

Salthouse, T. A. (1996). The processing-speed theory of adult age differences in cognition. Psychological Review, 103(3), 403-428.

Willcutt, E. G., Pennington, B. F., Olson, R. K., Chhabildas, N., \& Hulslander, J. (2005). Neuropsychological analyses of comorbidity between reading disability and attention deficit hyperactivity disorder: In search of the common deficit. Developmental Neuropsychology, 27(1), 35-78. 

Dankwoord | 171

\section{Dankwoord}


172 | Dankwoord 
De afgelopen vier jaar hebben veel mensen mijn pad gekruist en mij op professioneel en/of persoonlijk vlak bijgestaan en bijgedragen aan dit promotieonderzoek, proefschrift en mijn leven. In dit laatste deel van het proefschrift wil ik tot een aantal van deze mensen een woord van dank richten.

Beste Jelle Jolles, promotor, dank voor alle mogelijkheden en kansen die ik heb gekregen om kennis te leren maken met de wondere wereld van de klinische wetenschap alsook de geestelijke gezondheidszorg; dank voor het vertrouwen in mij om het ADHD-volwassenen project verder te hebben kunnen opbouwen, je enthousiasme en je bijdrage tot mijn professionele en persoonlijke groei. Beste Petra Hurks, co-promotor, dank voor de prettige samenwerking, ik heb er echt van genoten. Bedankt dat ik altijd bij je terecht kon met al mijn vragen en ideeën, je kritische blik op de zaken waar ik veel van heb geleerd. Bovendien heb ik je steun en interesse op professioneel en persoonlijk vlak altijd weten te appreciëren.

De leden van de leescommissie wil ik bedanken voor het beoordelen van dit proefschrift. Ook alle co-auteurs die hebben meegewerkt aan de onderzoeken beschreven in dit proefschrift wil ik bedanken voor hun belangrijke bijdrage en aangename samenwerking. Daarnaast wil ik Jurgen Cornelis en Lydia Krabbendam bedanken voor het voorwerk dat zij hebben geleverd in het opstarten van het ADHD-volwassenen traject op Vijverdal.

Mijn oprechte dank gaat tevens uit naar alle patiënten van de poli ADHD en proefpersonen die zich hebben onderworpen aan de studies in dit onderzoek; zonder hun belangrijke bijdrage had dit allemaal niet nader onderzocht en in de praktijk gezet kunnen worden. Ook wil ik alle behandelaren van de poli ADHD bedanken voor hun hulp en bijdrage in het onderzoek.

Alle (oud-)medewerkers van mijn directe werkplek op de Academische Afdeling Neuropsychologie ('AAN') en de poli ADHD van Vijverdal: bedankt voor alles en de prettige en gezellige werkplek! Vooral wil ik noemen: Brechje Dandachi-Fitzgerald, Carlein Karimoen, Desiree Op het Veld, Dymphie Scholtissen-Indebraek, Elvire Duchateau, Inge Knuts, Femie Kengen, Lilian Huijnen, Marielle Wetzels, Rudolf Ponds en Sven Stapert, die allen een grote steun en toeverlaat zijn geweest tijdens mijn onderzoeksjaren. Zonder jullie bijdrage in het onderzoek was dit 'boekje' nooit binnen deze termijn afgeraakt. Femie, die ik in het bijzonder wil bedanken om bij te dragen aan de lay-out van dit proefschrift.

Daarnaast wil ik ook alle (oud-) AIO's op mijn tweede werkplek op DRT 10 bedanken voor de steun, leuke praatjes in de wandelgangen en after-work feestjes die het AIO leven aangenamer maken. Speciale dank gaat uit naar mijn kamergenoten, Celeste Meijs, Inez Ramaekers, Heidi Lansdaal en Renske Wassenberg wil ik bedanken voor alle gesprekken over het wel en wee van een AIO en GZ-opleideling, tips en meedenken in het onderzoek. Lieve Celeste, je bent een bijzondere kamergenote voor mij geweest bij wie ik altijd terecht kon 
en veel lief en leed mee heb kunnen delen. Bedankt voor je luisterend oor, je vriendschap en het paranimfen.

Verder wil ik ook alle andere (oud-)collega's van Neuropsychologie en Psychiatrie bedanken waaronder Pascal van Gerven, Renate de Groot, Ron Keulen en Willemien Meijer om mee de vruchten te plukken van het IVO project. Pauline Dibbets en Katya Bakker en al de mensen van de fMRI scan afdeling van het aZM bedankt om mee een kijkje te hebben mogen nemen in de fMRI wereld. Mijn dank gaat ook vit naar Ankie Hochstenbach, Desiree Morales, Dick Willems, Elsa Misdom, Laurent Lowies, Marco van Hertrooy, Netty Buijs, Netty Ramaekers, Nico Roozendaal, Ron Mengelers, en Wim van der Elst voor al jullie ondersteuning tijdens de totstandkoming van dit proefschrift.

Ook wil ik mijn stagiaires, Angelique Gijsen, Climmy van den Nieuwenhof, Esther Steins, Loes Weusten, Mieke Vinkenrooye, bedanken voor al jullie hulp en enthousiasme. Tevens wil ik Eise Hendriks, Frederique Reiziger, Inge Verlinden, Krystle Penders, Mirjam, Jolle Jolles, Ron Keulen en alle stagiaires op de poli-ADHD bedanken voor hun medewerking bij het invoeren van de data. Zonder jullie hulp had ik het niet gered! Ook wil ik de mensen van de archivering op Vijverdal, in het bijzonder Maaike van de Reyen, bedanken voor hun hulp in het steeds aanleveren van de dossiers. Ook wil ik nog al mijn collega's van de GZ-opleiding bedanken voor hun steun en interesse in mijn promotieonderzoek.

Angelique Cachia, Jane Sykes en Sarah Stutterheim wil ik bedanken voor het corrigeren van mijn Engelse teksten en verschillende hoofdstukken.

Verder wil ik ook een aantal van mijn vrienden bedanken. Eerst en vooral Esmeralda Klinkenberg. Esmeralda, je bent een bijzondere collega voor me geweest waarbij ik veel heb kunnen lachen en af en toe kon uithuilen. Je blijvende steun en nieuwe invulling van lunch beginnen zijn sporen na te laten bij mij. Ik ben ontzettend blij om jou als paranimf en vriendin achter mij te hebben. Ook wil ik Josien en Hanneke bedanken voor de steun, pep-talk en luisterend oor tijdens het GZ-carpoolen, de Bakker Tony wandelingen en gezellige etentjes. Jullie zijn echt vriendinnen uit duizend en ik ben ontzettend blij dat ik jullie heb leren kennen. Ook wil ik al de mensen vit mijn familie en naasten bedanken die mij in al die jaren hebben gesteund.

Natuurlijk wil ik ook mijn ouders bedanken voor de onvoorwaardelijke steun en betrokkenheid. Mama en papa, jullie hebben de belangrijkste fundamenten voor me gelegd en me bijgestaan bij iedere belangrijke beslissing of als ik het even niet meer zag zitten. Bedankt voor alles wat jullie voor me hebben gedaan en dankzij jullie sta ik hier nu.

Tot slot, Gerrie, bedankt om er te zijn voor mij, je steun, en je zorgen en geduld als ik wel eens te lang kon doorgaan met werken. De ontspannende weekendjes en vakanties, je rust en vermogen om te relativeren zijn me dierbaar. 


\section{Curriculum Vitae}

Natalie Dominique Jenny Marchetta werd geboren op 22 december 1980 in Tongeren (België). In 1998 behaalde zij het diploma Latijn-Moderne talen aan het Koninklijk Atheneum te Tongeren. Hierna ging zij Psychologie studeren aan de Universiteit Maastricht. Als afstudeerrichting koos zij voor Biologische psychologie met als afstudeervariant Neuropsychologie en zij deed in haar laatste jaar een klinische stage aan het Openbaar Psychiatrisch Ziekenhuis ('OPZ') te Rekem (België). Vervolgens rondde ze haar wetenschappelijke stage gecombineerd met een klinische stage af bij de Academische Afdeling Neuropsychologie ('AAN') van het Psychomedisch Streekcentrum Vijverdal. In mei 2003 behaalde ze haar diploma. Aansluitend werd zij als Promovendus aangesteld bij de capaciteitsgroep Psychiatrie en Neuropsychologie aan de Universiteit Maastricht. Haar werkplek was de afdeling Neuropsychiatrie/ Poli ADHD van het PMS Vijverdal waar ze tot juli 2007 werkte. Het onderzoek dat zij hier deed staat beschreven in dit proefschrift. In januari 2005 begon zij tevens parttime de opleiding tot gezondheidszorgpsycholoog op de afdeling Psychiatrie en Neuropsychologie van het Academisch Ziekenhuis Maastricht en op de poli ADHD van het PMS Vijverdal. Sinds 1 augustus 2007 is zij bezig met het verder afronden van deze opleiding bij de groepspraktijk Virenze te Rijckholt. 
